\title{
The mutable dynamics of HIV-prevention: Insights from Ontarian MSM on pre-exposure prophylaxis (PrEP)
}

\author{
By \\ Adam Christianson
}

A thesis submitted to the faculty of Graduate and Postdoctoral affairs in the partial fulfilment of the requirements for the degree of

\author{
Master of Arts \\ in
}

Sociology

\author{
Department of Sociology \\ Carleton University \\ Ottawa, Ontario
}

(C) 2017

\section{Adam Christianson}

All rights reserved. This thesis may not be reproduced in whole or in part, by photocopy or by other means, without the permission of the author. 


\begin{abstract}
Pre-exposure prophylaxis or "PrEP," is a cluster of methods that keep HIV-negative persons from contracting the virus. Effectively PrEP allows members of "high risk" communities a form of immunity against the virus. This thesis examines how PrEP is interpreted by men who have sex with men (MSM) PrEP users and non-users from south-east Ontario. Comparing the accounts of MSM users and interested non-users of PrEP, I examine barriers to implementation and how PrEP interacts with established methods of sexual hygiene and prophylactic preference. I examine how HIV-stigma is challenged by PrEP and challenges PrEP's viability as a technology. I discuss how a successful implementation of PrEP as a mainstay of HIV prevention technologies stands to significantly impact HIV stigma and allows for forms of intimacy not afforded by current HIV technologies or safer sex practices.
\end{abstract}




\section{Acknowledgements}

While my name is the only one that will be connected to this project from now on, this project like all others, would not have been possible without the hard work of a substantial support network. I would like to take this opportunity to thank all my colleagues, professors, friends and family for encouraging and supporting me over the last two years.

In particular, I would like to thank my supervisor Carlos Novas for his patience, encouragement and advise. Thank you to my colleagues for their support, theoretical contributions and comments on my drafts. Thank you, Zoe Jones, for introducing me to literature on edgework and intimacy that revolutionized my thinking about prophylactic use and intimacy. A special thank-you to my father and volunteer editor, Tod Christianson for his unending support and his assistance with editing and review. Thank you Marsha Rosengarten and Michael Mopas for your helpful and insightful questions at my defence. Thank you Janet Siltanen and Alexis Shotwell for your help developing my methods and theory respectively. Special thanks to the support and administrative team, without whom I would have been lost. Thank you Paula Whisell and Xiaobei Chen for your guidance and support.

I would like to thank the institutions that supported my project. Thank you to the Gender and Sexuality Resource Centre and the Pride Centre/Centre Fierté for supporting my project and for allowing me to use your space. A big thank you to Andy Simmonds of Hey Rooney, for letting me use his art free of charge. I would like to thank the Social Sciences and Humanities Research Council, the Province of Ontario and the Department of Sociology and Anthropology for funding my graduate studies, without which this project would have been impossible.

Finally, I would like to thank my wonderful participants for taking the time to share their opinions and experiences. I hope this project will be of use to you. 


\title{
Glossary of Terms and Acronyms
}

\author{
Bareback (noun/verb) The sexual practice of engaging in condomless anal sex. \\ Gay (noun)
}

Grindr (noun)

GRID (acronym)

HAART (acronym)

Highly Active Antiretroviral Treatment

Heterosexual (noun)

Refers to persons with an enduring pattern of opposite gender attraction. See also: Straight

Homosexual (noun)

MSM (acronym)

Refers to persons with an enduring pattern of same gender attraction, usually used in a medical context. See also: Gay

MSM is the medical term for any male-identified person who engages in sexual activity with another male-identified person, regardless of sexual identity. MSM is meant to reference practices, rather than any specific community.

Poz (noun)

Slang for HIV-positive

Queer (noun/verb)

Queer has come to be the accepted term for the umbrella of sexualities and gender identities otherwise described as LGBT. It is generally used in reference to mainstream heterosexuality and/or cisgender persons.

Seroconvert (verb) The point in time where HIV becomes first detectable. 
Serosort (verb)

Straight (noun)
Serosorting or to serosort is the practice of selecting whether or not to engage in condomless sex based on a partner's HIV status relative to their own HIV status.

Colloquial for heterosexual. See also: Heterosexual

Table of Contents

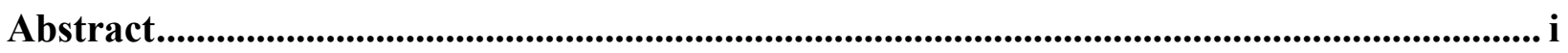

Acknowledgements ........................................................................................................................ ii

Glossary of Terms and Acronyms ......................................................................................ii

Chapter 1 A Magic Pill or Just Magic Beans? ................................................................................. 1

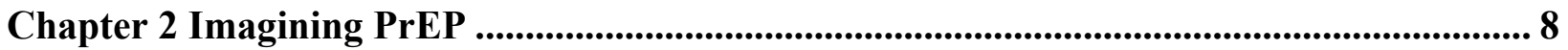

Affordances and Interpretations.................................................................................................9

PrEP Use as Interpreted by Other Academics .....................................................................................14

Inscription and Interpretations: Multiple Risks and the Flexible Interpretations of PrEP ...........19

Resistances and Reformulations: Non-Humans and Subjectivity ......................................................24

Assemblages .........................................................................................................................................28

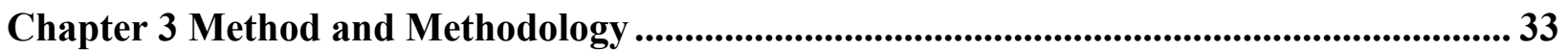

My Approach to and Method of Instrumentation.....................................................................................33

Sampling Strategy and Inclusion Criteria ………............................................................................35

Questionnaires .................................................................................................................................................36

Why Questionnaires .....................................................................................................................................36

Questionnaire content .........................................................................................................................................37

Focus Group Content ....................................................................................................................................40

Ethics Approval ....................................................................................................................................41

On Naming ....................................................................................................................................................42

Data Analysis Strategy: The Listening Guide ……..........................................................................43

The Readings.........................................................................................................................................44

The Analytic Power of the Listening Guide .........................................................................................47

What Cannot be Known .........................................................................................................................49

Chapter 4 (Mis)configuring the User: Barriers to Access and the Configuration of the PrEP User as "At Risk" ............................................................................................................................ 51

Barriers to Use Identified in the Literature ......................................................................................52

Barrier: The cost of treatment...................................................................................................................55

Barrier: Access to Sympathetic and Knowledgeable Health Care Practitioners ..............................61

Barrier: Stigma, the Disinhibition Hypothesis and the \#TruvadaWhore .............................................70

Barrier: Side Effects and Weight Gain ..................................................................................................77

Is PrEP Configured Correctly?...................................................................................................................79

Chapter 5 Prophylaxis and the Mutability of HIV Risk ........................................................ 82

PrEP Use: As a Complement to Condoms ........................................................................................84

PrEP Use: As a Replacement for Condoms ............................................................................................87 


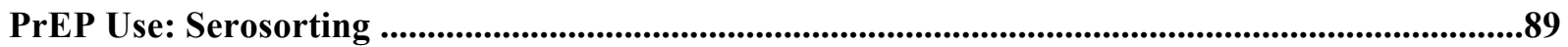

The Multiple Constructions of HIV Risk .................................................................................................96

The "Latent Need for PrEP" "......................................................................................................................103

Chapter 6 The Changing Constitution of the HIV Assemblage ......................................... 109

Changing Practices: Immunitary Solidarity and HIV-Risk ..................................................110

Changing Practices: PrEP use and Obtaining Consent .........................................................118

The HIV Assemblage: Constructing Queer Identities ..........................................................................127

Revisiting the "Latent Need" for PrEP: A Political Promise ......................................................135

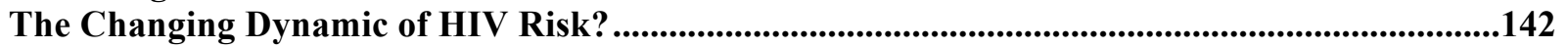

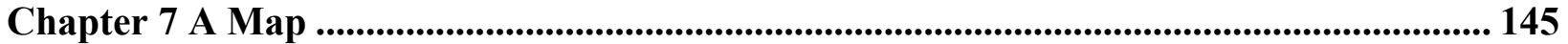

Limitations and Areas for Further Research ...................................................................149

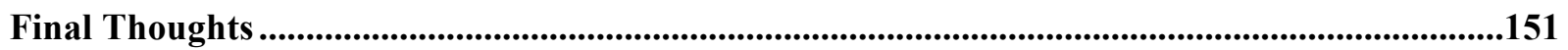

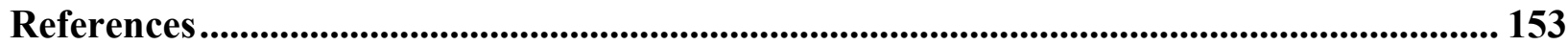

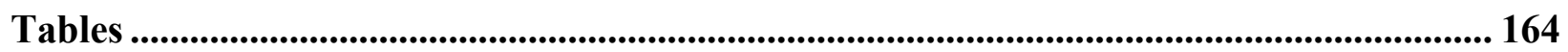

Table 1. A typology of appropriate safer sex techniques according to relationship type ..............164

APPENDIX A. User Online Questionnaire ......................................................................... 165

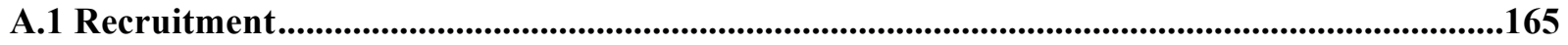

A.2 User Consent Form and Questionnaire Pack............................................................................167

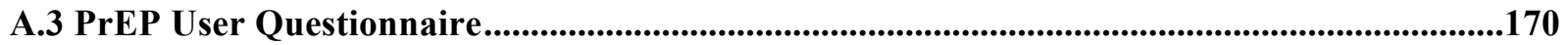

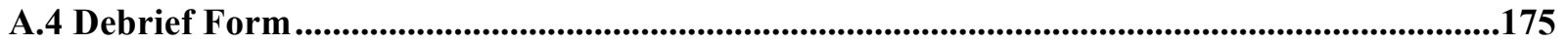

B. Non-User Focus Group ....................................................................................................... 177

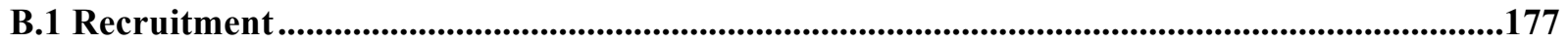

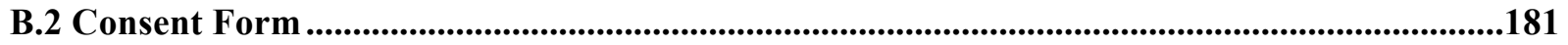

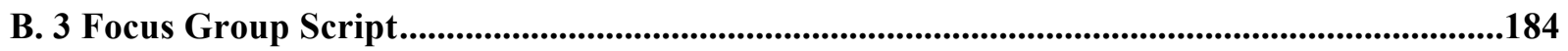

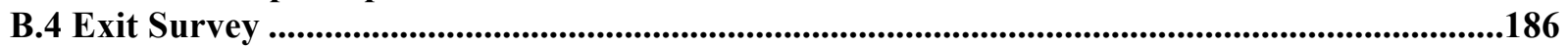

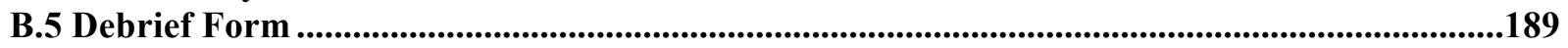

C. Analysis Strategy .................................................................................................................. 192

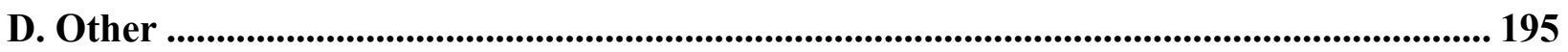




\section{Chapter 1}

\section{A Magic Pill or Just Magic Beans?}

"I think the idea of [PrEP] being a "magic pill" for sex to return sounds great!"

-Focus group participant 008, PrEP non-user

My first day of taking Truvada for PrEP was emotional. I got dizzy. After decades of fear of HIV, of even associating gay with death, there was a path to overcoming the plague. It was all coming together. ART and PrEP were redefining what HIV management and prevention meant. We could conquer HIV and overcome our fear, shame and despair. There was a way to bring together poz [HIV-positive] and negative people. Negative women could have children who wouldn't be infected by their poz undetectable partner. Now I was part of the experiment on the PrEP side, proudly.

-Christian, PrEP user

The impact of HIV in North America is undeniable. At an average rate of 2000 diagnoses annually, 80,000 people in Canada have been diagnosed with HIV since 1985 (Public Health Agency of Canada, 2014). Though, technically, any person anywhere could potentially be infected, men who have sex with men (MSM) have seen the lion's share of HIV diagnoses. First imagined as a "gay plague," HIV has spread to communities beyond the MSM communities so closely associated with the disease. After 35 years, what was once known as the "gay plague" has been reimagined. Nonetheless, the association of queer sexuality with HIV is cemented in health provision, in safer sex practices and in AIDS discourse that framed HIV as a "gay disease." In the $21^{\text {st }}$ century, four decades since the initial outbreak, HIV continues to impact queer communities disproportionately and thus prevention efforts continue to require us to at least consider the possibility that either we or our partner may be infected.

How does the assumption of infection bear on the relationships between these persons, and what would negating that assumption do to those relationships? How would we relate to each-other, if HIV was not a universal, perpetual and perhaps inevitable risk? What would 
happen if we introduced a highly effective pill that allowed us to remove HIV from these calculations. How would people receive that pill? How would we think about ourselves, our families, our friends, our lovers and those already infected knowing that we were immune to such a devastating virus? How would those not taking this "magic pill," as one of my participants called it, react to those who were taking it? Would this pill expose where HIV the virus ended and HIV stigma (and the various inequalities inherent in that stigma) begin?

This thesis addresses how such a pill might impact relationships between MSM in Ontario. In the last 5 years, advances in prevention technologies have shown promise for significantly decreasing the prevalence of HIV in "high risk" populations_-including MSMs. The focus of this thesis, Truvada, is a combination antiretroviral medication, patented by Gilead Sciences. Previously used to treat HIV, Truvada has recently been approved to prevent HIV in HIV-negative persons at high risk of infection. These medications, known as pre-exposure prophylaxis or "PrEP", allow one to hamper the spread of the virus in the body, if exposed. What is particularly interesting about PrEP, compared to other forms of HIV-prevention, namely condoms, is that PrEP's function as a prophylactic occurs by changing the nature of the cells in the body, as opposed to creating a barrier. Truvada as PrEP functions by disabling the HIV virus from replicating inside the cell. This functional quality of PrEP creates new possibilities for how people interpret their HIV risk and thus also offers new interpretations of what constitutes safer sex.

In trying to understand how PrEP impacts these relationships I attempt to understand how it is accessed and ultimately used by MSMs. By comparing the accounts of PrEP users and interested non-users, I attempt to uncover who accesses PrEP, for what reasons, and what barriers exist that might render PrEP less effective at preventing the spread of HIV than clinical 
studies might suggest. I examine how PrEP is understood in relation to other prophylactic technologies and practices used by MSM to minimize exposure to HIV, and try to understand how HIV risk evaluations might be impacted by PrEP. In answering these questions, I investigate the nature of HIV stigma as both a barrier to PrEP use and something to be overcome by using PrEP. Finally, I consider the long-term implications of PrEP, should it become a mainstay of HIV prevention techniques. Though significant barriers to communities of use remain, and there is still a possibility that PrEP may remain a niche technology, users and non-users alike seem to understand the transformative possibilities PrEP might offer; such as for enhancing queer solidarity and changing the nature of queer intimacy.

Truvada for PrEP has been found to be extremely effective at reducing HIV seroconversion in serodiscordant couples and in at risk communities. These studies indicate that PrEP averages an effectiveness of $95 \%$ across studies (Jiang et al., 2014). Though there are signs that PrEP, when used as indicated, is highly effective at preventing HIV transmission (e.g. Chelsea and Westminster Hospital, 2017), barriers to use and potentials for misuse certainly stand to impact whether or not PrEP will become a mainstay of HIV prevention techniques. The way the drug changes the meaning of sex and safety, the boundaries of the body as well as the stigma related to sex are all affected by shifts in the network of artefacts and social groups that make up the cosmos of HIV. Though unquestionably effective, PrEP has yet to stand trial in the real world. Though one might imagine a pill that seems to virtually eliminate the risk of transmitting HIV would have resounding positive effects, PrEP is entering a massive and complex system of relationships where it may cause unexpected harm.

The next two chapters of this thesis examine how PrEP is imagined and how those discussions have impacted how I think about PrEP. Chapters 2 and 3 examine the literature and 
theory that inform my thinking about PrEP as well as the method and methodology used in this thesis. Chapter 2 outlines how I attempt to bridge discussions of technology, risk and queer life with existing literature on PrEP. HIV prevention is multiple, lively and complex. We can imagine HIV as multiple interacting dynamic systems in which persons, groups and objects, who might not seem to be affiliated with HIV prevention at times surface in unexpected relationships. I argue that PrEP as a successful prevention technology enters this system as a new structure, changing the overall composition of the system itself and impacting these relationships in all sorts of interesting ways. PrEP is entering a complex system of interrelated actors and actants with established norms, and tacit agreements and time honoured prevention efforts. Upon entering this system, PrEP is already being interpreted by groups who have different understandings of HIV, prevention and how PrEP ought to be used. Each of these interpretations of PrEP signal emergent social groups with at times irreconcilable perspectives on HIV. How these networks of relations between men who have sex with men are changing as a result of PrEP, how are they themselves changed by PrEP and how these changes affect the way HIV is understood and ultimately transmitted is key to HIV prevention efforts.

This thesis examines how PrEP challenges established norms and practices developed to mitigate HIV risk. PrEP impacts the system of social grouping and exclusionary practices developed around serostatus. The HIV epidemic divides queer guys along their HIV-status or "serostatus," allowing a system of morality to develop around the virus and viral transmission pathways. For many, a 'responsible' gay man is also an uninfected gay man. Gay men manage their risk of HIV, not simply by keeping the virus outside their bodies but by keeping socially unsanctioned sexuality outside of the body. Non-heteronormative forms of sexuality are especially stigmatized and are associated with being "high risk". In this sense unprotected anal 
sex, or "barebacking," is equated with actively seeking infection. This, however, does not necessarily disassociate notions of what it means to be contaminated from more established ways of thinking about infection. Cells inside the body can be all but infected, but this does not mean that the body is necessarily polluted. How does one negotiate their sexuality when this boundary between infected and uninfected is both invisible to the naked eye and blurred by chemophylaxis? How does one maintain their identity as responsible and/or uninfected when they are exposing themselves to the virus; knowing that although the virus permeates their cells, it will not reproduce enough for them to be designated as 'infected?'

This paper draws from recent developments in science and technology studies (STS), including recent interventions from queer and feminist theory. I treat PrEP, as well as a number of other human and non-human actors as assemblages. When I use this term, I mean dynamic systems of open and interrelated parts, the ontological status of which is neither static or permanent, whose arrangement in space and time gives rise to a particular subjectivity. How these moving parts interact and play off each other allows us to understand the role of PrEP in the relationships of, largely "gay" Ontarian, male-identified persons. Thus, understanding how this assemblage is moving (or at least how it might move) can help us understand, predict and change the spread of HIV in light of its implementation. I maintain that PrEP is in relations of co-formation with the groups that use (and some that do not use) it. In attempting to understand these relations, this paper attempts to answer three broad questions: What barriers to use might stop PrEP from becoming a successful HIV intervention? how might PrEP impact relations between and within queer communities, and, should PrEP be successful, how might it change the way queer men imagine their bodies and selves? 
In chapter 3, I discuss how I obtained my data from PrEP users and non-users and how using an analytical strategy called the listening guide the data were analysed. In order to understand how PrEP stands to impact the relations of men who have sex with men in Ontario, I ventured to glean insights from members of this community. Because I am interested in emergent interpretations of the drug created by queer persons, and because information about the drug is sparse across MSM communities, I opted for an information oriented sampling strategy. In order to balance inclusivity with efficient data gathering, I sampled adult queer guys according to usership. This method takes into account the perspectives of two groups of people who represent the maximum variation of personal experience with PrEP in the queer community. This paper uses data from both PrEP users and non-users to better understand the relations that might exist within and between each group. The first data source comes from questionnaires sent out to queer guys who have recently undergone or are currently on a PrEP regimen. The second data set comes from focus groups with persons who are not on PrEP. The transcripts from these findings were then coded using an adapted form of narrative analysis called the listening guide. My findings are a conversation between these two groups of data.

The chapters that address my findings move from the most immediate problems facing users and potential users to the more abstract implications of PrEP as a mainstay of HIV prevention. This analysis first examines how potential users are excluded from becoming users. Chapter 4 examines which "barriers" to use are identified by my participants, barriers that keep PrEP usership disproportionately low. From there I move on to how PrEP impacts established norms around sex and sexuality. In chapter 5 I investigate how PrEP is resisted and reformulated according to how participants imagine PrEP in relation to other prophylactics and risk mitigation strategies. I investigate how my participants envision how PrEP changes their understanding of 
condom use and the system of negotiated condom use known as serosorting. I discuss how PrEP disturbs the system of relations that make up HIV risk in ways that may ultimately encourage a separation of HIV stigma from queer sexuality. These shifts are already apparent in user and non-user's discussions of solidarity, trust and consent. In chapter 6, I discuss how PrEP stands to shift how queer men organise, how they negotiate consent and how they envision solidarity. On one hand, it appears PrEP offers a way to overcome the "viral apartheid" (Dean, 2009) of HIV status. On the other hand, this may only be possible if a corresponding shift in norms around prophylactic use is instituted. If that is the case, it is certainly possible that PrEP will fundamentally shift how queer guys understand themselves. 


\section{Chapter 2}

\section{Imagining PrEP}

I draw largely from science and technology studies to accomplish the analysis I have sketched above. I argue that PrEP is an actant with specific capacities, capacities that facilitate relationships that are not possible using other current forms of HIV prophylaxis. PrEP revolutionizes how persons interact with HIV. As PrEP interacts with HIV on the molecular level, it allows a person to interact with sexual partners as if they were immune. Though academics who have previously studied PrEP agree that PrEP is highly effective, they offer several scenarios where PrEP may be ineffective and in fact may be harmful.

A novel and arguably unique HIV prevention technology, Truvada has been the subject of much speculation about whether it is designed properly, as it is the first prophylactic of its type widely available. Some have argued that PrEP has been poorly configured, relying on a configuration of the ideal user as either vulnerable or irresponsible and thus requiring additional protection from HIV (Holt, 2015; Rosengarten \& Michael, 2009; Beaten \& Grant 2013; Rowniak \& Portillo, 2013). While many academics studying PrEP argue that PrEP may cause a paradoxical increase in HIV transmission due to treatment optimism, it is also the case that PrEP may not lead to the reduction in HIV diagnoses seen in clinical trials due to this mischaracterization of the ideal PrEP user. Indeed, concerns from potential users tend not to be preoccupied with a "disinhibition hypothesis." Rather, queer persons in prior studies seem far more interested in avoiding stigmatization and controlling their HIV risk than actually having any more sex than they were at the time.

I argue that PrEP is poorly defined and currently being interpreted by "target" communities as they weigh their HIV risk with the risk that they might be labeled a "Truvada 
whore." Though access is certainly a pressing matter with respect to PrEP and HIV prevention, how PrEP impacts with the broader relationships that make up queer life and HIV prevention requires attention. As PrEP enables novel ways of interacting with HIV to HIV-negative persons, which groups manage to integrate PrEP's afforded uses within their particular system of sexual norms will likely result in emergent communities from within these established queer communities. In other words, PrEP may signal dramatic a shift in how queer sexuality is understood. This chapter concludes with a brief discussion of how I attempt to understand PrEP's emergence in networks of materiality and meaning that one might call the "HIV assemblage."

\section{Affordances and Interpretations}

In order to understand how PrEP use changes relationships between queer persons, it is first important to understand how PrEP interacts with HIV in HIV-negative bodies. I argue that PrEP's mechanism of action, how it engages with HIV, offers novel ways of relating to HIV and HIV-positive bodies. I use the term "affordance" to describe how artefacts interact with humans and other non-humans through a non-human's unique characteristics, usually derived from the artefacts physical, chemical or biological capacities. Affordance is a term, first coined in environmental psychology, that attempts to limit the variations in interpretations of an artefact to those that are traceable to the materiality of the artefact. By delimiting the possibilities for interpretation to only those offered by the artefact, Gibson (1979) endeavored to offer an alternative to Gestalt theorists who posited that the utility of an artefact is in the eye of the user and thus infinitely variable. Gibson argues that while the interpretation of the affordance is variable, that affordance remains constant (Hutchby, 2001, p.448). 
Hutchby (2001) argues that affordance is useful to sociologists studying technology. He argues that it offers a compromise between technological determinism and social constructivism. According to Hutchby (2001) an affordance is both the functional aspects of a technology that enables as well those which constrain activity. That is to say, while a technology can be somewhat flexible in its interpretation, aspects of the materiality of the artefact constrain how that artefact can be interpreted. Focusing on the afforded uses of a technology alloys the analyst to attend to the possible interpretations offered by a non-human, without giving primacy to either the human or the non-human.

Differences in the interpretation of a technology, Hutchby (2001) argues is due to the fact that artefacts are employed in "complex sets of concepts and conventional rules governing their use" (449). As I explain below, both PrEP and condoms interfere with HIV and thus make them viable and potentially effective HIV prophylactics. However, how both of these artefacts afford prophylaxis is drastically different, and because they interact with HIV in such drastically different fashions, it is unclear if PrEP will ultimately be intelligible as a prophylactic. Because condoms have a three-decade head start on PrEP as an HIV prophylactic it has been enmeshed in the rituals that make up both HIV prevention, but also other aspects around sexuality, such as consent. Thus, whether or not PrEP will become a mainstay of HIV prevention techniques is both due to whether or not it effectively halts HIV reproduction as well as whether or not it intelligible as a prophylactic as defined by the customs that constitute HIV prophylaxis.

Akrich and Latour (1992) have offered several terms to help describe these types of relationships. Briefly, they define engagements with non-humans as interactions between actors (humans) and actants (non-humans), the latter of which have capacities that allow them to be meaningfully engaged in a particular social situation or a "performance." These actants are 
"active" in relationships through their affordances. Akrich and Latour (1992) explain that the raw capacities of an artefact can be harnessed by designers to impart messaging upon or inspire behaviour in other humans and thus helps to order events. Latour (1992) uses the example of the Berliner key, a key that functions only by pushing the key back through the lock where it remains in place until unlocked, to explain how the physical qualities of the key and lock imparts the message "RELOCK THE DOOR BEHIND YOU" (253). A script is the "social life" or semiotic quality of a non-human, related to but not necessarily the intended effect of a characteristic of a non-human. In the case of PrEP, it is the capacity to interfere with viral DNA that allows it to be intelligible as an HIV prophylactic for persons at substantial risk of contracting HIV. Of course, as this thesis aims to explore, these affordances make available several scripts, depending on the goals of the relevant interest groups involved.

With these definitions in mind, let us briefly discuss how PrEP interacts with HIV and how it affords use as an HIV prophylactic as a result of that interaction. A standard PrEP ${ }^{1}$ treatment in Canada consists of a daily regimen of one oral tablet containing $200 \mathrm{mg} / 300 \mathrm{mg}$ of emtricitabine and tenofovir disoproxil fumarate (Gilead Sciences, 2016). PrEP is indicated to be used with condoms to reduce the risk of sexually transmitted HIV in high risk groups.

The component drugs, emtricitabine and tenofovir, function as anti-viral medications by inhibiting the reverse transcription of HIV RNA in infected host cells. Both substances are part of a family of drugs called nucleoside reverse transcriptase inhibitors (NRTIs). These drugs are "nucleoside analogs;" meaning that they are similar in shape and composition to the building

\footnotetext{
${ }^{1}$ From here on I will refer to Truvada as "PrEP." It is worth nothing that there are a number of other forms of pre-exposure prophylaxis currently being tested that are also part of this group of methods. There are also generic forms of Truvada such as Tenvir-EM in circulation, though Tenvir-EM it is not currently approved for use in Canada.
} 
blocks of RNA or DNA. Because of their similar shape, emtricitabine and tenofovir obstruct the reproductive cycle of HIV by interfering with the formation of new viral DNA. Once inside the cell, the virus is dissolved and releases its RNA into the cell to be converted into usable pieces of DNA. It accomplishes this translation, first by converting its RNA into viral DNA that is then spliced into the DNA of CD4+ T cells, where it then proceeds to use the cell to build new HIV virions. Emtricitabine and tenofovir halt reverse transcription by terminating this process. The HIV reverse transcriptase enzyme does not have a mechanism to distinguish between normal nucleotides and non-functional analogs like Emtricitabine or Tenofovir. These DNA chains are incomplete and thus unable to instruct the cell to construct new HIV virions, thereby halting the progression of the infection in the cell and the spread of the infection to other potential host cells. PrEP's mechanism of action offers novel ways of interacting with HIV and thus might enable novel performances that were not available using other forms of prophylaxis. PrEP and condoms are the only current HIV prophylactic methods available to HIV-negative persons in Canada. Condoms function by creating a barrier between a potentially infected body and an uninfected body, allowing one to keep pathogens from moving from one body to the next. PrEP on the other hand functions as a prophylactic by changing the nature of the cells in the body. These two prophylactic methods allow for very different engagements between sexual partners, potentially broadening the safer sex methods available to those who, for one reason or another, do not use condoms.

There is reason to expect that Truvada might become a mainstay of HIV prevention. While Truvada has only been conceived of as PrEP for a little over 10 years, and in use in North America for about 5, the component drugs are well established and trusted by medical practitioners the world over for treating HIV. Tenofovir is an established antiretroviral drug, 
listed by the WHO as part of the essential medicines that make up the "minimum medicine needs for a basic health-care system" (World Health Organization, 2015a). It is known to be a safe drug (Grant et al., 2005; Kojima \& Klausner, 2016; Desai, Iyer \& Dikshit, 2012) that is well tolerated by most users, with minimal side effects (Beaten \& Grant, 2013; Grant et al., 2005; Kojima \& Klausner, 2016). It is preferred as a "first line" drug due to a "favorable pharmacokinetic profile," easy oral absorption, with or without food, and because it has a low risk for drug interactions (Panel on Clinical Practices for Treatment of HIV Infection, 2002). Finally, the intracellular half-life of tenofovir makes it useful as a daily pill and might also offer some protection if a dose is missed (Grant et al., 2005). Emtricitabine is equally well established and is also listed as a minimal requirement for any basic health care system, as a complement to tenofovir (World Health Organization, 2015a). Although not all clinical findings were equally positive, both preclinical findings and clinical trials indicate the potential for PrEP to be an effective HIV prophylactic (Beaten \& Grant, 2013).

In addition to being a well-established HIV treatment, initial findings in clinical studies of Truvada as PrEP are promising. Studies of PrEP boast an average effectiveness of 95\% across studies (Jiang et al., 2014). A recent study has found that it is no more harmful, in the short to medium term, than aspirin (Kojima \& Klausner, 2016). It is lauded for its ability to reduce a person's viral load to nearly undetectable levels; reducing the risk of transmission to sexual partners by as much as 95\% (Young, Flowers \& McDaid, 2016). Against a placebo, PrEP was found to contribute to between $42 \%$ and $85 \%$ decrease in seroconversion in at-risk groups (Grant et al., 2010; Baeten et al., 2012; Molina et al., 2015). There are indications that PrEP has already accounted for as much as a $42 \%$ decrease in HIV diagnoses in London hospitals (Chelsea and Westminster Hospital, 2017). 
If these initial clinical findings are any indication of PrEP's effectiveness as an HIV prevention technique, its wide spread implementation could shift HIV from a pandemic impacting millions of people around the world to a far more manageable illness. Unfortunately, despite strong evidence that it is effective, PrEP has been received unevenly members of target communities. Persons who use PrEP report being shamed for taking PrEP and have come to be known in some circles as "Truvada whores" (Speildenner, 2016; Calabrese \& Underhill, 2015; Belluz, 2014). Other community members are concerned that this prevention method may lead to over confidence in a person's relative HIV risk, leading to an increase in HIV transmission rather than a decrease (Race, 2016). For this reason, it is imperative to further clarify which relationships and practices are impacted by PrEP in order to adequately respond to the changing dynamics of HIV transmission in Ontario.

The divisive nature of PrEP offers an excellent opportunity to uncover the shifting dynamics of use and resistance experienced by queer persons at risk of contracting HIV. Focusing on how PrEP is interpreted by conflicting groups within the MSM community, as well as how it is interacting with the broader HIV prevention assemblage allows us to understand how viable PrEP might be as a prevention technology. By comparing the interpretations of PrEP by current users and non-uses we can further understand why people want to use PrEP. These accounts can help further understand how users interpret PrEP and the networks of relations that are changing as a result of PrEP use. These frames tell us as much about the users, the non-users and the social life of HIV as it does about the pill itself.

\section{PrEP Use as Interpreted by Other Academics}

Truvada is a well-known HIV treatment but has only recently been conceived of as a prophylactic since the mid-2000s. Understandably, there is a far larger body of work on the 
potential implications and ethics of HIV chemophylaxis than there is work on the interpretation of PrEP by user communities. While studies of how users experience PrEP is increasing rapidly, these experiences are not yet well understood (Young, Flowers \& McDaid, 2016). How nonusers and users experience a PrEP mediated sexuality is currently underexplored and, most importantly, does not comprehend the social effects of the drug in practice (Knight et al., 2016). This is confounded by recent findings, though PrEP is relatively well known among gay men, the actual take-up is fairly low (Goedel et al., 2016; Hull, Edward \& Kelley, 2016; Bush et al., 2016; Hoots et al., 2016). Why is a drug that is so effective at preventing HIV and about as harmful as an aspirin so under accessed?

Some have noted that low uptake could be a result of a mischaracterization of the PrEP user. As opposed to a drug that treats a specified illness, PrEP is designed to respond to a probability of infection, rather than an infection itself. As a result, PrEP has been indicated for those at the highest risk of contracting HIV. It is perhaps for this reason that PrEP is highly controversial (Young, Flowers \& McDaid, 2016; Holt, 2015; Rosengarten \& Michael, 2009). How does one justify their "high-risk" status while maintaining they are not in fact a danger to themselves or others? Thus, PrEP is a highly divisive treatment, not just for medical practitioners, who are afraid PrEP use might lead to miscalculations of HIV risk (Beaten \& Grant, 2013; Desai et al., 2015; Rowniak \& Portillo, 2013; Underhill \& Meyer 2013; Spinner et al., 2015; Young, Flowers \& McDaid, 2016; Speildenner, 2016), but for members of the target community who are increasingly polarized on whether or not PrEP users are responsible members of society, let alone desirable partners. From the perspective of some queer men, PrEP is a tool for some guys ("Truvada Whores") to pay to eschew well established norms around sexual hygiene. In other words, is PrEP really being used as an HIV prevention drug for "at risk" 
groups or is Truvada just another 'male enhancement' drug, masquerading behind HIV prevention?

Though this thesis is largely preoccupied with the debate above, biomedical approaches to PrEP have also pointed to a number of ambiguities in design that might lead to the ultimate failure of PrEP as a viable HIV prophylactic. These scholars tend to group around a few main concerns: The potential for antiviral resistance (Tellalian et al., 2013; Rowniak \& Portillo, 2013); patient non-adherence and the paradox of increased transmission related to "treatment optimism" (Tellalian et al., 2013; Beaten \& Grant, 2013; Desai et al., 2015) and the potential for disinhibition or increased risk taking associated with an increased sense of safety for those taking the drug (Beaten \& Grant, 2013; Desai et al., 2015; Rowniak \& Portillo, 2013; Underhill \& Meyer 2013; Spinner et al., 2015; Young, Flowers \& McDaid, 2016; Speildenner, 2016). Beaten and Grant (2013) further note that it is not currently known what constitutes the optimal dose, ideal dosing frequency and when to start or end a PrEP regimen. There has been a great deal of difficulty anticipating frequency and timing of intercourse in addition to body type and the time it takes for Truvada to reach concentrations required to effectively enable HIV prevention (Hull, Edward \& Kelley, 2016). Additionally, it is not entirely clear who ought to be termed high risk and ideally suited for PrEP (Beaten \& Grant 2013; Rowniak \& Portillo, 2013; Holt, 2015) Finally, Rhodes and Wong (2016) stress the need for further research assessing the meaning of sexual relationships and how more concrete sexual practices such as condom use and biomedical interventions are defined in relation to more abstract concepts such as love, trust, and intimacy (194). They maintain that HIV prevention should consider the ways different relationships are built and maintained by several of the diverse MSM constituent communities. 
At the time of writing, the biomedical and public health literature on PrEP forms a more substantial body of literature than those examining the social implications of PrEP. Despite the growing number of studies on PrEP, they continue not to consider the social-structural factors that might affect the efficacy of PrEP in the real world (Roberts \& Matthew, 2012). Inadequate understanding of the social patterns that affect the spread of HIV have led to the failure of other HIV interventions in the past (Kingori \& Sariola, 2015). To avoid placing PrEP on a trajectory that ultimately fails to further hamper the spread of HIV, I endeavor to study the role of the social relations that bear on PrEP, in addition to the social-structural, biomedical and behavioral arguments that already predominate PrEP literature (Rosengarten \& Michael, 2009b; Roberts \& Matthews, 2012).

This thesis aims to build on these suggestions by comprehending how PrEP is engaged with existing relationships between queer guys in Ontario. PrEP has the potential to change the network of relations that make up the HIV epidemic, but only to the extent that it is resistant to redefinitions by competing understandings of use. These notions include both, how queer guys might understand themselves in relation to the inscribed "ideal" PrEP user generated by designers, distributers and medical practitioners, as well as how potential users might interpret alternative uses for pill. This decision to use, resist using or redefine using PrEP is made in part in relation to notions of risk and responsibility. Notions that are unevenly taken up by queer guys. Broadly, a 'responsible' gay guy is an uninfected (HIV-negative) gay guy. Gay guys manage their risk of HIV, not simply by keeping the virus outside their bodies but by keeping "filth" outside of their bodies. Filth, in this sense, can be defined as the breaching of barriers by things that a given society perceives as impure (Hacking, 2003; Douglas, 1966). Filth then includes the HIV virus but also requires keeping socially unsanctioned sexuality outside of the 
body. Non-heteronormative forms of sexuality are especially stigmatized and are associated with being "high risk," regardless of the actual likelihood of passing or contracting HIV. Following this logic, unprotected anal sex or "barebacking," is equated with actively seeking infection, regardless if one is taking appropriate precautions or not. Until recently, the technological and social relationships conducive to HIV infection have been relatively stable, allowing for the establishment of norms around condom use and HIV testing that allow persons to manage their HIV risk.

The conflation of sexuality and HIV risk is also a problem for medical practitioners. PrEP use is problematic for practitioners because it conflicts with their obligation to provide treatment in a manner that is beneficial to their well-being" with "the moral obligation to not inflict harm on others" (Rowniak \& Portillo, 2013, p.8). As HIV risk in North America is defined not only as a disease spread through the contact of body fluids containing virions or infected cells, but also according to a distinct epidemiological pattern of "male homosexual intercourse or contact with infected blood" (Porter \& Kaplan, 2011, p.1602), medical practitioners are required to consider both the community in which the patient is engaged as well as their patient's individual risk profile. PrEP thus brings into conflict a health practitioner's desire to protect the individual bodies of patients with the obligation to protect the public from disease.

The concern that is most often brought up by health researchers and medical practitioners when discussing PrEP is the conflict between the desire to help patients who would benefit from PrEP, while avoiding harming the larger social body. They fear that by providing PrEP to a person who might use the pill to engage in ever riskier sex, they might contribute to increasing the spread of HIV (Rowniak \& Portillo, 2013). This paradox, more commonly framed by health researchers as the risk of 'disinhibition' (Rosengarten \& Michael, 2009b, Dennin, 
2016) constitutes a major problem for both potential users and for HIV prevention planning and policy. While the uncertainty around changes in HIV transmission fomented by PrEP are notable, there is the very real possibility that denying a person PrEP based on this risk is itself a harm to those who are truly at risk of contracting the virus. There is no immediate or easy solution to this question for practitioners trying to determine appropriate courses of action relative to the risk involved.

\section{Inscription and Interpretations: Multiple Risks and the Flexible Interpretations of PrEP}

In attempting to comprehend how PrEP impacts the broader network of relations that make up the HIV assemblage, it is important to understand how it may interact with the communities that stand to be impacted most by a change in HIV transmission. In order to do so, one must first understand how PrEP stands to interact with pre-existing dynamics of risk, dynamics that are particular to the queer sexualities that stand to benefit from PrEP. In other words, how does HIV risk come to be understood by and for MSMs and how does PrEP impact those understandings of risk? The next section addresses literature dealing with how PrEP is a novel technology constructed in relation to HIV "risk groups" and how that interpretation stands to impact PrEP. The next section addresses the relationship between HIV, HIV prevention technologies and how these technologies have changed the network of social relations for queer sexualities in the past.

The future of PrEP as an HIV prevention technology is complicated by Gilead's construction of PrEP as a drug for persons "at risk," without much additional detail in the configuration of the ideal user. In the Truvada product monograph, Gilead Sciences (2016) specifies an ideal user for their product. The company outlines the ideal PrEP user in terms of their biological characteristics as well as the socio-sexual practices that are most likely to benefit from Truvada as PrEP. The concept of the 'technological script' is particularly useful in 
imagining the prescribed user of Truvada as PrEP (Holt, 2015). Akrich (1992) defines a technological script as "an attempt to predetermine the settings that users are asked to imagine for a particular piece of technology and the prescriptions that accompany it" (208). The concept of script is useful because it denotes a projected user (Schot \& de la Bruheze, 2003) or a group that is requested to fill a particular role (Akrich, 1992) that is either filled, left empty or redefined by the corresponding user group. As Truvada is, to some extent, rendered unintelligible or "black-boxed" by the time it reaches medical practitioners and, ultimately, the user, this could mean that PrEP may already be destined to fail or may be reconfigured in the near future.

The extent to which PrEP can adapt to the social world in which it emerges is key to its success as a preventative technology. This has to do with the accuracy and flexibility of its script to the communities that might benefit from Truvada. The extent that Truvada is obdurate closes out particular interpretations of it as anything other than its scripted use. At the same time the success of Truvada as PrEP relies on the accuracy of this script to its corresponding intended user group. An analysis of research publications found that characterizing the ideal user of PrEP as at "high risk" for HIV, without further configuration has led to an uneven reception and some ambiguity about who effectively uses PrEP (Holt, 2015). This analysis found that although framing PrEP as a drug for people who are "vulnerable, in need, at high risk of HIV [infection] and in tangential connection with services...helps justify making PrEP available" (ibid, 436) but leaves PrEP intertwined in the dynamics of risk and responsibility that have helped shape the post-HIV outbreak gay community (Flowers, 2001).

The Truvada product monograph does not focus on the social identities or the communities of users, but rather conforms indications along the same lines as those established by Health Canada. These indications focus on the ideal physical bodies and the risk factors that 
make a person an ideal PrEP user. To receive this treatment, an individual should fit the following criteria, irrespective of the community to which they belong. According to the product monograph, a person is suited for Truvada as PrEP in the following cases:

If the individual has partner(s) known to be HIV-1 infected, or

- engages in sexual activity within a high prevalence area or social network and one or more of the following:

- inconsistent or no condom use;

- diagnosis of sexually transmitted infections;

- exchange of sex for commodities (such as money, food, shelter, or drugs);

- use of illicit drugs or alcohol dependence;

- incarceration or

- partner(s) of unknown HIV-1 status with any of the factors listed above (Gilead Sciences, 2016, p.3)

These recommendations closely follow the risk categories used by Health Canada for HIV surveillance (Public Health Agency of Canada, 2014). Other than these practices, however, the Truvada product monograph does not attempt to offer any additional configurations of the ideal PrEP user. According to Gilead, Truvada is only useful as PrEP for HIV-negative individuals who are also at increased risk of contracting HIV. Though framing PrEP as a drug for basically any person who is at "substantial risk" of acquiring HIV has helped justify making PrEP widely available, this definition misunderstands many of the social realities facing potential PrEP users (Holt, 2015). The development of PrEP as a product targeted to "vulnerable" or "high-risk" individuals, in fact, offers a narrow interpretation of the kind of person who may benefit from PrEP. Holt (2015) argues that this current configuration is not simply poorly defined, but carries with it an implicit bias that PrEP users are either vulnerable or irresponsible. Descriptors, he argues which are certainly not the case for many PrEP users.

For potential PrEP users, this vague definition poses a problem. Joshua Edward, for example, notes that the queer guys who visit his sexual health centers routinely misunderstand 
their HIV risk as either higher or lower than their behaviour might indicate (Hull, Edward \& Kelley, 2016). These risk calculations do not necessarily line up with those on the side of health care practitioners, who are instructed to prescribe PrEP to MSMs at substantial risk of HIV acquisition (World Health Organization, 2015b) that includes those with inconsistent or no condom use and/or a history of STIs (Centers for Disease Control, 2014; Hull, Edward \& Kelley, 2016). This partial configuration of the ideal PrEP user according to nebulous categories such as MSM and "at substantial risk," pose a threat to PrEP's efficacy as an HIV prevention strategy. As opposed to most drugs that are designed to treat an illness, PrEP interacts with its target illness as a prevention technology. PrEP has been forwarded as a drug ideally suited to high risk individuals. By offering otherwise healthy persons a drug framed as a risk-mitigation strategy, PrEP engages with queer sexuality through its relationship with HIV risk. Broadly, prevention is a form of risk management where the surveillance of factors that lead to unfavorable outcome are identified, such that appropriate actions can be taken to prevent that unfavorable event from occurring (Rabinow, 2005; Rose, 2007; Hacking, 1990). Rabinow (2005) argues that modern prevention efforts focus not on the individual but on the interpersonal factors that make a risk possible. Through the surveillance of likely occurrences, "deviant behaviour" can be minimized, and "healthy" behaviour can be maximized. These risks are not "specific dangers posed by the immediate presence of a person or a group which make a risk probable" (Rabinow, 2005, p.187) but are depersonalized practices or other factors that make a risk likely. Because HIV spreads through particular practices and has a well understood reproductive cycle, HIV risk can be managed by adapting sexual practices to be lower risk and/or by interfering with the HIV reproductive cycle. HIV, however, also has a long history of being associated with particular groups, whose characteristic practices happen to parallel the most efficient routes of 
HIV transmission and thus makes sexual orientation a factor to be surveilled alongside other "deviant" behaviours.

An effect of this conflation of sexual identity with risk factors is already apparent in responses to PrEP by both medical practitioners and members of target groups likely to benefit from PrEP. Although medical practitioners tend to frame barriers to the effectiveness of PrEP in terms of disinhibition, young gay men tend to be more preoccupied with the potential misinterpretation of PrEP use and 'avoiding stigmatization' (Kubiek, Arauz-Cuadra \& Kipke, 2015; Frankis et al., 2016). Young, Flowers and McDaid (2016), for example, found that their participants were "highly attuned" to HIV stigma and how access to knowledge about PrEP and TasP would interact with that stigma. Specifically, that the existing sero-divide (between those who were HIV-negative and positive) shapes the knowledge available to either group and might negatively impact whether or not potential PrEP users access knowledge about PrEP. HIVnegative persons actively distanced themselves from HIV as a way mitigate that risk (Young, Flowers \& McDaid, 2016; Keogh, 2008).

Stigmatization is one of the more substantial risks that aim to impact PrEP's role in HIV prevention. The emergence and circulation of the term "Truvada whore" to stigmatize persons taking PrEP demonstrates additional considerations that need to be made by potential users as part of their risk profile (Speildenner, 2016; Calabrese \& Underhill, 2015; Belluz, 2014). A recent example from the literature points to the complexity of these risk calculations. Although respondents in a study of Scottish gay men seemed to think that PrEP may help strengthen the bonds between serodiscordant partners, their tone shifted to the negative when discussing how their own partners might react to learning they were taking PrEP. Respondents were concerned that PrEP, even the presence of a medication in their homes might "scare" their partner, or that 
their partner might misinterpret PrEP use as a sign that they are in fact HIV-positive (Kubiek, Arauz-Cuadra \& Kipke, 2015 p.491). Addressing the question of whether PrEP will be a successful intervention involves understanding how HIV risk manifests in a stigma that may serve to include or exclude particular types of users.

It is important, therefore, to understand how PrEP is interpreted as a technology by intended users, as well as those not explicitly defined by the script. How PrEP includes, excludes, or is resisted by its ideal users is key to understanding the potential future(s) of PrEP as a preventative technology. Therefore, considering the trajectory of a technology necessitates an understanding of the evaluations of that technology made by users and non-users (Watkins, 2011). In technology studies, studies of users far outnumber studies on non-users perhaps due to the sense that non-users are presupposed to either be irrelevant or excluded (Wyatt, 2003), however, as Watkins (2011) notes, the active rejection of a technology has implications for the success of a technology, especially in cases where stigma and risk are involved. Thus, in my discussion of HIV risk mitigation strategies, understanding the parallel experience of non-users and users can help us understand the trajectory of PrEP in the near future in Ontario.

\section{Resistances and Reformulations: Non-Humans and Subjectivity}

Though it is a matter of debate whether or not non-humans are intentional in interactions, it is certainly the case that non-humans afford relationships according to their particular characteristics. These qualities are conducive to specific functions and are thus configured such that they are useful for specific types of performances (Akrich \& Latour, 1992). Though the setting in which HIV is contracted may be variable, HIV interacts with the body in a fairly regular fashion. Its mechanism of engagement with cells in the body is regular enough to be surveilled and treated. This constellation of "at risk" communities, health providers, testing 
technologies, pharmaceuticals, and the disease itself form the HIV prevention assemblage. This assemblage includes the code for human performances, including but not limited to a person's sexuality. It is the relations within this assemblage that PrEP impacts. Collin (2016) argues that pharmaceuticals increasingly serve as "major vectors" of a transformation in our collective understandings of the world. She describes pharmaceutical use as a dispositif, or the variable constellation of actors (and actants) moving with no explicit direction or directionality (75). Dispositif are easier to understand through their effects than their "cumulative outcome, but is less clearly seen by the various - and sometimes contradictory - agents working through it." (ibid).

HIV helps defines how we understand our sexual practices. Though we do not necessarily think about our sex exclusively or even consciously through the lens of HIV risk, our sexual practices are nonetheless framed in relation to HIV. Though queer sexualities are multiple, variable and transient, we have little difficulty delimiting men who have sex with men (MSM) from other forms of sexuality. Though the United Nations notes that a sizable proportion of MSM identify as heterosexual, and may indeed be married, we lump together all men who engage with each-other sexually in the name of HIV risk and prevention (Joint United Nations Programme on HIV/AIDS, 2006). Indeed, for our purposes, it is worthwhile to operate using MSM as a term in its own right. On the one hand, because as I will argue, PrEP problematizes MSM as a concept at all and because MSM, by and in large should be targeted by health care professionals as the site where PrEP would be most useful ${ }^{2}$. Reducing the number of HIV

\footnotetext{
${ }^{2}$ Health Canada notes that Injection drug users (IDUs) and MSMs make up $63 \%$ of new HIV diagnoses annually (Public Health Agency of Canada, 2014).
} 
diagnoses in this surveillance category would result in a substantial decrease in HIV diagnoses overall in Ontario.

The 'MSM' exposure category has been criticized for its role in the erasure of the experiences of gay men (Young \& Meyer, 2005) and has been argued to be part of discourse that could limit the effectiveness of PrEP (Spieldenner, 2016). Nevertheless, MSM is reflective of the current manifestation of relations related to the management and surveillance of HIV transmission in Canada (Bowker \& Starr, 1999; Health Canada, 2015) from which we must begin this analysis. As such, I have attempted to limit my analysis to those who might fit in this this exposure category ${ }^{3}$. As Bowker and Star (1999) point out, standards are more than cognitive devices for understanding the world, standards set the course for the material manifestation of those categories in the world. Indeed, epidemiological categories form a self-reinforcing loop between specific communities and disease (Mol, 2001). Therefore, when studying the relationship between practices and HIV-risk, it is inadvisable to frame this discussion without some consideration of the medical interpretation of same-sex sexuality. Though MSM does not adequately understand the richness and variability of the communities that fall under its umbrella, these categories are both a result of past developments in the history of the HIV epidemic and continues to shape the way we understand HIV transmission in Canada.

Though the epidemiological categories and communities impacted by PrEP reinforce each other, there are several social and technological systems involved in the management of the HIV retrovirus that influence how we understand HIV risk and as a result frame our inclusion in these communities of risk. As Flowers (2001) demonstrates, technological innovations over the

\footnotetext{
${ }^{3}$ For clarification on how I use "queer", "MSM" and "gay" please refer to the glossary of terms above.
} 
last 35 years have reframed the community that is commonly referred to as 'Gay men.' Since the first discovery of elevated cases of Kaposi sarcoma in gay men, those impacted by HIV have been fractured and redefined by developments in HIV prevention and detection technologies at least three times (ibid). For example, the invention of HIV-Blood testing is a diagnostic tool that helps manage HIV risk which it also functions to divide Gay men the lines of HIV risk (ibid). Before that time, HIV risk was directly related to one's identity or 'lifestyle' as a Gay man. The shift from referring to HIV/AIDS as GRID to its current manifestation as human immunodeficiency virus and acquired immune deficiency syndrome (HIV/AIDS) is a result of the shift in understanding brought about by the discovery of the virus (ibid). The discovery of the virus and the development of techniques for detection created the possibility for our current classifications of HIV risk.

While it is certainly the case that technological innovations have changed how we act in relation to HIV, it is important not to fall victim to technological determinism. Watkins (2011) points to the substantial role users and non-users play in determining the ultimate success of an intervention. Her study of the contraceptive technology, Norplant, bears many similarities to PrEP. Touted as a "very effective" (39) novel contraceptive technology aimed at persons who did not have regular access to contraception, Norplant never managed to reach the success of other contraceptive technologies (Watkins, 2011). In particular, cost, side effects and fears of disinhibition and the potential for rising STI rates were all debates that bore on the development and implementation of Norplant as a contraceptive technology. These debates that are echoed in challenges to PrEP, many of which are outlined above. Despite these more obvious issues related to implantation and use, Watkins (2011) argues that the ultimate failure of Norplant rested on the decisions made by that "spectrum of users and non-users" (37) of this contraceptive technology. 
Germane to PrEP is the finding that potential users of Norplant made their decision to become a non-user, not based on their objective contraceptive needs, but rather on "personal and social experiences mediated by 'oral networks' of information exchange with friends and family” (45). Watkins (2011) explains that these networks were informed by a growing association at the time between Norplant and moral panics around 'welfare moms' and female sexual child abusers. In a similar vein, PrEP users are subject to what AIDS activist Peter Stanley calls "gay on gay shaming" most notably resulting in the label "Truvada whore" (in Belluz, 2014; see also Speildenner, 2016). How PrEP users and non-users respond to this construct remains to be seen.

Alternatively, Flowers (2001) notes that relevant social groups reinterpreted preventative technologies to sort sexual partners. Though originally meant to help survey HIV transmission and provide treatment for those infected, HIV testing simultaneously created a system of categories based on serostatus (HIV-positive, HIV-negative and unknown status). This antibody

test thus became a tool used by the some of the various social groups under the MSM umbrella to cater their condom use. Following the introduction of the HIV test, a shift in the "boundaries of risk" occurred, moving risk from all gay men to kinds of gay men (i.e. Those at risk and those who are a risk) (56). This shift also necessitated a change in behaviour around sex and sexuality as gay men were serosorting. This practice has been criticized as creating a system of "viral apartheid" between infected and uninfected gay men (Dean, 2009). In this sense, it becomes increasingly clear that HIV technologies and the impacted social groups are implicated in dynamic networks of co-formation.

\section{Assemblages}

In order to think through this dynamic, I propose to understand PrEP as an assemblage, (sometimes referred to as a "network," "ensemble" or any series of multiple dynamics of co- 
formation) as the most generative approach to understanding its effect on our target community(ies). Broadly, an assemblage is the dynamic system of open and interrelated parts the ontological status of which is neither static nor permanent and where the specific arrangement of actors and actants in space and time gives rise to a particular subjectivity. I argue for this approach as the most generative for several reasons. The most important reason being that it allows me to give due attention to humans and non-humans in a network with a reasonable amount of flexibility to describe the varying levels of agency available to those actors/actants in time and space. While taking into consideration the lived experience of 'being at risk,' it allows an analyst to resist identity as taken for granted, stable or singular. This is particularly useful in addressing the role of identity, power, and technology as we investigate PrEP's emergence in HIV prevention practices and in sexuality more generally.

This paper addresses the emergence of PrEP, a prophylactic assemblage, within two other overlapping networks: the queer body/self as an assemblage and the HIV assemblage. For this reason, we will discuss 3 different dimensions of sociality which are in conversation with PrEP. The analysis that follows attempts to understand how PrEP engages with these two other interrelated networks. Approaching PrEP in this way allows us to embrace the complex network of relations that form around sexuality, HIV, and HIV prevention as they bear on PrEP, while avoiding giving primacy to any.

Markus and Saka (2006) argue that assemblage as an organizing concept offer an "antistructural" account of structure-like phenomena. It allows the analyst the ability to discuss emergent phenomena within ordered systems without relying on traditional notions of structure and agency. Social constructivists in STS have argued for an anti-deterministic approach such as this one to understand the role of non-humans in the social. Akrich and Latour (1992) suggest 
reading interaction with technology as, "assemblies of humans and non-humans where the competencies and performances are distributed" in a particular setting. By interacting within these "messy" networks (Bijker, 2010), actors orient themselves relative to those networks. Through their interpretation of the non-human, humans enter into "alliances" with technologies (Callon, 1984; Law, 1987; Latour, 1992) and through those alliances impart meaning upon things otherwise uninscribed. Considering the role non-humans play in the social is especially important when discussing PrEP, as understanding it requires us to understand the social implications of both a drug and disease on the subjectivities of those impacted by the virus. Further, understanding how disease and immunity are constructed through non-humans like PrEP and HIV requires us to investigate the semiotic quality of matter. In order to do this, I have found assemblage to be the most suitable organizing concept to understand the layers of meaningful relationships that make up HIV and HIV prevention.

Understanding the body as part of an assemblage allows us to avoid giving primacy to cultural, technological, or biological determinants of a person's subjectivity. Rather, it allows us to attend to the semi-sable relations between human and non-humans that in colliding, make up an intelligible "event" (Parisi, 2004; Puar, 2007). Persons implicated in the event are "mutated" by these configurations, both in a metaphorical and sometimes physical sense (Parisi, 2004, p.28). Thus, rather than understand experience as the confrontation of forces or relations of domination by a "stable," positioned self, an assemblage attends how those forces produce or inhibit a person's ability for movement in time, space and in dimensions of sociality.

An assemblage approach understands non-humans and humans as interacting in and on each other on various points of scale (Venn, 2006). These persons and the technologies they are using are reciprocally formed in, by and with the environments and encounters with the persons 
with which they are engaged. This perspective allows us to understand the infinite variability and richness of experiences that occur across seemingly identical events or interactions. It seems that this perspective allows us to create transformative, politically relevant explanations of events that are still of practical use to health care practitioners. Understanding how the patchwork of humans and non-humans, in tangible and intangible relationships of varying levels of awareness, come to make meaningful divisions among people who, otherwise identify similarly, is key to understanding the ways HIV will continue to propagate itself among these persons and how the dynamics of HIV risk might be changed.

Finally, understanding how PrEP interacts in, with, on and within queer communities allows us insight into its role in changing the body, identities, power structures and "life," more generally. Though all technology changes how we live our lives, biomedical technologies, like pharmaceuticals, are acutely interesting because they enact change in the biological function of our bodies themselves, changing life itself. PrEP is interesting because it disturbs notions of immunity. By interacting with HIV at the molecular level, it shifts the site of infection from the cell to the DNA inside the cell itself. As a result, we can begin to imagine novel notions of contagion, prophylaxis and infection that may arise because of this transformation.

This analysis focuses on PrEP from three perspectives. The first chapter focuses on PrEP itself; as Rosengarten and Michael (2009) note PrEP is an ontologically open medical intervention that extends beyond the pill itself into the relations of matter and meaning that make it either an effective or ineffective intervention in HIV. Focusing on how PrEP use is currently defined, I discuss how queer guys negotiate becoming a user to understand how its design implies a specific user, that is either taken up or resisted by potential users. The second chapter addresses the practices that make up same-sex sexuality and discusses how participants interpret 
PrEP's intervention in those changes. From this perspective, we discuss how PrEP interacts with other more established rituals around sexuality to understand how PrEP is taken up and resisted by queer guys. I argue that PrEP has multiple contrasting uses based on a person's sexual preference. These contrasting uses point to how PrEP stands to redefine which practices are considered risky and makes clear tacit stigma in how HIV risk is understood by queer and nonqueer persons alike. The final chapter investigates how PrEP might stand to redefine the relationships that co-form queer identities and our understandings of HIV risk. Each chapter thus examines a part of a greater whole to give us insight into the complex interplay of actors and actant that make up the HIV epidemic in the early $21^{\text {st }}$ century. 


\section{Chapter 3 Method and Methodology}

This thesis attempts to understand how the networks of relations between men who have sex with men are changing due to the introduction of PrEP. It remains unclear how PrEP is being taken up, by whom and for which reasons. Additionally, how PrEP interacts with commonly held beliefs about infection, risk and which sexual partners/practices are acceptable remains unexplored. In order to understand how these dynamics are changing, I gathered information from both persons who use PrEP and who currently do not use PrEP. This chapter outlines the way the data used in this thesis was collected from both of these groups, and how it was analysed. I explain which data collection methods were used and why they help us understand these networks. I argue why it is important to consider the input from both users and non-users of PrEP in determining its technological trajectory. In the final section of this chapter I discuss my data analysis strategy, the "listening guide," and describe the type of data this strategy generates. The chapter concludes with a discussion of what can and cannot be known using this data analysis strategy.

\section{My Approach to and Method of Instrumentation}

In order to clarify the delimitations of my conclusions, it is important to briefly explain the nature of those conclusions. In order to draw qualified inferences from relatively small numbers from a specific population, I approach method by drawing on the notion of phronesis (Flyvbjerg, 2001). Phronesis originates in Aristotle's theory of knowledge, specifically that social sciences deal in knowledge of things that are "good or bad for [humanity]" (Flyvbjerg 2001, p.57). As opposed to positivistic knowledge, popular in the natural sciences, phronetic knowledge is not interested in the production of theory in a universal sense. Rather, phronesis is 
knowledge useful to those interested in how one ought to conduct themselves in a particular context (58). This form of knowledge is gathered from "lay experts," in order to uncover power dynamics and answer value-rational questions that pertain to guiding the direction of a particular social group (59). By attending to a form of knowledge that attempts to answer, "what is good", we are able to more clearly describe social relationships and answer social problems that are often framed in value-rational terms. This version of social science offers an important complement to the study of HIV and approaches to HIV prevention, that often speak in terms of population and can sometimes shy from questions of power (Mykhalovskiy, 2010). By approaching the study of PrEP in this way I am able to more clearly attend to how PrEP and the HIV prevention assemblage are co-forming.

Following previous work on PrEP, this thesis attempts to understand how sets of associations of drugs and bodies acts with or against the current techniques of HIV prevention (Rosengarten \& Michael, 2010). This focus on how various elements coalesce to produce a meaningful engagement or event is coming to be a theme in the study of PrEP. This perspective is particularly useful as it is apt to understand how humans and non-humans come together to create a meaningful encounter that simultaneously changes both of them (Race, 2016). As the aim of this thesis is to understand how HIV prevention is enacted in light of the implementation of PrEP as part of a range of interventions into the sexualities of HIV-negative persons, it is key to this analysis to understand exactly how this prevention is done. On the other hand, focusing on how PrEP is resisted and accepted, through an analysis of the stories of insiders, can help us understand how queer persons create systems of meaning that structure their relationship with those on PrEP. These perspectives when put together contributes to a further understanding of the ethics and practices of common sexual practices as they take shape in the near future. 


\section{Sampling Strategy and Inclusion Criteria}

This project draws its conclusions from two main sets of data. The first data source comes from questionnaires sent out to queer men who have recently undergone or are currently on a PrEP regimen. The second data set comes from focus groups conducted with persons who are not on PrEP. The following section describes the two processes, the sampling strategy and the composition of each group sampled.

Participants were sampled according to an information oriented strategy that most closely aligns with the "maximum variation" style of participant selection (Flyvbjerg, 2001; Patton, 2002). According to Flyvbjerg (2001), the purpose of information based case selection is to do targeted sampling of relatively small groups on the grounds that those smaller samples have a particular information content (79). Maximum variation style sampling draws its analytical power from the significance of a number of comparable circumstances for case process and outcome (ibid). Participants are normally selected on the basis of a significant difference along one or more dimension. In this case, participants were selected along the lines of PrEP use. Selecting participants according to difference is especially useful in diverse samples where great heterogeneity would otherwise lead to unproductive results (Patton, 2002). In this case, the purpose of my sampling strategy was to narrow down my sample to groups with either a high or a low level of experience with the drug.

The questionnaire and focus groups take into account the perspectives of two groups of people who represent the maximum variation of personal experience with PrEP in the queer community. Participants were allowed to participate in the questionnaire portion of the study so long as they were adult men who have sex with men who lived in Ontario during their time on 
PrEP. The non-user focus group was open to any adult (18 years of age or older) who is interested in PrEP and who was also HIV-negative.

\section{Questionnaires}

I sampled users from online communities of people who have or who are currently taking PrEP, with an interest in sharing their experiences. Though I posted invitations on forums such as Tumblr and Reddit, I received responses from members of Facebook groups for those interested in PrEP, exclusively. These websites are the most efficient space to recruit users, as they are most likely interested in sharing their experiences. Once identified, participants were provided with a questionnaire package that they were asked to complete at their leisure. This questionnaire package contained an informed consent form, the questionnaire itself and a debrief package (see appendix).

In total, I received six completed questionnaires. As a group, my sample of PrEP users was fairly homogeneous. Most participants were professionals between the ages of 25 and 54 . Five participants were white, one was black, and most had a combined household income of more than $\$ 80,000$ yearly. Two participants have been on PrEP for over two years, one under two years and two have been taking PrEP for under a year. One of these participants has stopped taking PrEP at the time of response. Just over half my respondents identified as single, while the rest described themselves as "married or similar." All my participants lived in the eastern and central regions of Ontario and mainly resided in the Greater Toronto Area or Ottawa while they were on PrEP.

\section{Why Questionnaires}

As PrEP users are located all over Ontario, and belong to a hard to reach population, the most efficient method of data collection was to sample users from online communities interested 
in PrEP. Opdenakker (2006) notes that computer mediated interviewing techniques are useful for hard to reach and geographically sparse populations, are amenable to studies with relatively open-ended questions or unstandardized interviewing, and can help ensure the confidentiality of respondents. Using digital questionnaires sent over email allowed me to reach PrEP users across southern Ontario.

Online recruiting is also useful in that these tactics are less time sensitive and can occur asynchronously. This allows respondents to answer at their own pace. This has positive implications for enabling participants to think through and control their narratives and reasonably manage any potential harms that might be overlooked in a face-to-face interview. The only substantive challenge to this approach is that the affective responses of the participant are lost, including any embodied social clues or emotional expression (Opdenakker, 2006). This challenge, however, can be addressed to some extent by using Doucet and Mauthner's (2008) "listening guide" to determine which points are emotionally laden.

\section{Questionnaire content}

The questionnaires discuss two key areas of PrEP usership: becoming a user and negotiating sexuality while using PrEP. The questionnaire first asks participants to outline how they learned about PrEP and how they managed to acquire a prescription. It then asks them about how PrEP has changed their sex lives and their relationships more generally. The questionnaire attends to key interpersonal experiences, in general, between the PrEP users, their peers and with their partner(s). I asked participants to discuss their views on condomless sex, or "barebacking," and whether or not their view has changed since taking PrEP. Participants were encouraged to write 250 words or more in response to each question; paying attention to the timing of the event, the actors involved, the progression of events and to reflect on their affective experience of that 
event. The intent of the questionnaire is to allow participants to provide a sufficiently contextualized narrative of events as they were experienced by the participant.

\section{Focus Group}

Focus groups are an efficient way to gather a great deal of information from a large number of non-users simultaneously (Morgan, 1997; Stewart, Shamdasani \& Rook, 2007), thus minimizing the chances that an interview will be unproductive when exploring a relatively unknown or novel subject. Focus group interviewing brings together groups of unrelated individuals for group discussion on one or a number of topics, in hopes of uncovering social or psychological constructs related to the researchers question of interest (Berg, 2009). This method of data collection is useful for exploratory research and when there is relatively little known about the phenomenon of interest to the researcher (Stewart, Shamdasani \& Rook, 2007). As opposed to individual interviews, focus groups allow for the collaborative generation of data that can unearth ideas and opinions that might not be uncovered in a traditional interview (Stewart, Shamdasani \& Rook, 2007). Further, focus groups create a complex, unique social situation that allows the researcher to understand how a diverse number of persons understand and interact with a phenomenon and allows for dominant discourses to emerge between participants, without necessarily being prompted by the researcher. This data collection strategy, therefore is especially useful when sampling groups that have highly variable levels of experience with the topic being discussed. Morgan (1997) notes that the comparisons amongst participants makes focus groups a valuable source of information, especially when a researcher is interested in shared or diverse understandings of an object (15). Though non-users are more plentiful than users, they are less likely to be aware of the details of PrEP use than users and thus are less likely to offer a detailed account of the relationships inherent in PrEP on their own. 
Sampling of non-users was done at the two largest universities in Ottawa. Universities were chosen as the most efficient location for recruitment as it would likely attract MSM of university age. ${ }^{4}$ I deemed this age group to be the most efficient group to sample from as they are at a higher risk for STI transmission and are more likely to be interested in safer sex techniques than members in the general public. Universities are spaces with high volumes of foot traffic and were, therefore, more likely to attract persons interested in safer sex topics such as HIV prevention techniques. To enhance the likelihood of participation, one focus group was held on each campus, in the gender and sexuality student centers.

Non-users were invited to a focus group and PrEP information session via posters posted in various locations across two university campuses in Ottawa. Though it was heavily implied that the focus groups were targeted to MSMs, the invite did not specify any particular target demographic. Participants were allowed to self-select so as to attract persons whose personal experience made PrEP a salient object. This sampling method is informed by Flyvbjerg's (2001) assertion that social knowledge is context dependent and that respondents are intuitively aware of their social environment, though not always consciously so.

In total two focus groups were conducted. The first focus group was made up of four persons and the second was comprised of five. Compared to my sample of PrEP users, the nonuser group was more diverse and younger. My focus groups, though largely populated by MSMs, did attract transmen and cisgender women. As I advertised exclusively in universities, the age range is reflective of persons typically thought to be university aged (Dale, 2010). Every one of my respondents were under 45 years of age, the vast majority of which fell between the ages of 18 and 25. All but two identified as “white." Understandably, as I had sampled from a university,

\footnotetext{
${ }^{4}$ The most likely of a university student is between 18 and 25 (Dale, 2010). People between these ages are also at the highest risk for contacting an STI (Public Health Agency of Ontario, 2015).
} 
every participant was in the process of completing a university degree. One participant had a mix of college and university education. The household incomes reported by non-user participants was far more inconsistent than those of the user group, ranging anywhere from a household income of under fifteen thousand to greater than one hundred thousand. Only two participants were single, the rest identified as married or similar.

\section{Focus Group Content}

Focus groups eased participants into discussing PrEP, first by familiarizing them with the drug, allowing them to reflect on the pill before moving on to they felt about interacting with PrEP users. I first introduced participants to the drug by presenting a fifteen-minute power point presentation on PrEP. This presentation outlined what PrEP is, its mechanism of action, how it is used and the process one would have to undergo should they choose to start taking PrEP. Following this presentation, participants were asked about their perceptions of PrEP. Participants were prompted to outline exactly what makes them "excited" about PrEP as well as things that concern them. Finally, participants were introduced to three hypothetical scenarios, where participants were asked to imagine how they would act in scenarios involving a person taking PrEP (see appendix).

I chose to use hypothetical scenarios in order to facilitate discussion about what for some is a sensitive topic. As HIV-risk is a potentially difficult subject to discuss in a public setting, opening up the floor to a hypothetical scenario is both productive and allows my participants to test the social norms around HIV as opposed to discussing their own personal choices. Hypothetical scenarios, or "vignettes," offer a practical solution to discussing potentially sensitive topics. Given the sensitivity of safer sex and the potential for stigma associated with 'unsafe' sexual choices, framing a question in the form of a vignette, as opposed to asking about 
a person's sexual practices outright, gives the participant the option to discuss their opinions in a hypothetical situation. This is useful as it allows for a certain level of depersonalization and thus allows participants to avoid exposing themselves to the group unnecessarily (Schoenberg \& Ravdal, 2010).

Vignettes were left fairly short to cut down on different interpretations, allowing rather for those details to emerge through discussion organically. I made this choice in order to preserve the contextual nature of the focus group. I allowed the participants the agency to interpret the question and collaboratively construct a scenario, rather than impose one derived from theory that might be resisted by my participants. In this sense, these questions rest on abductive as opposed to deductive or inductive approaches to knowledge generation. As opposed to inductive or deductive reasoning, abductive research does not rest on the confirmation or genesis of a single hypothesis, but rather focuses on the contextually driven and collaborative approach geared toward solving a puzzle (Schwartz-Shea \& Yannow, 2012). Constructing questions in this way acknowledges the experience and expertise of participants as experts in their experience of navigating their social environment (Flyvbjerg, 2001). Thus, leaving the question open to interpretation allows participants to make the question their own, allowing them to generate possibilities that can be otherwise curtailed by hypothetical scenarios that try to manufacture an experience.

\section{Ethics Approval}

This project was cleared by the Carleton University Research Ethics Board-A (CUREBA) at Carleton University, August $19^{\text {th }}, 2016$, following minor revisions. Despite being identified as minimal risk by the ethics office, this project was sent to a "full board" review. The reason for full board review were due to concerns about the vulnerable status of HIV infected gay men were 
raised as well as and my insistence that users were given the option to choose whether or not they wanted to use their real names.

\section{On Naming}

My participants have varying levels of anonymity. One will notice that my participants are at times identified by name and at other times are numbered. This is due to the varying levels of anonymity made possible by the mixture of methods employed in this study. Participants who completed the questionnaire were given the option to use their name or choose a pseudonym. Though questionnaire respondents were given the option to choose a pseudonym, non-users who participated in the focus groups were not given this option. Focus group participants were automatically assigned a number based on their position around the table. How participants are named relates, largely, to the methodological reasoning behind how that data was generated.

Methodologically, one of the most important assurances of quality in qualitative research, is maintaining transparency and building trust between respondents and researcher (SchwartzShea \& Yanow, 2012). This is all the more pressing in research that deals with personal narratives. By giving my user participants the agency to choose their name and allowing them to craft their own responses, I am hoping to give them a sense of ownership, thereby building trust. By shifting the power to choose the identity of the respondents back to the respondents I am both giving agency to my respondents as well as ensuring the integrity of their narrative.

As Lahman and colleagues (2015) argue, one should take the name (or the pseudonym) of the participant very seriously. As they write, "it may be argued a rose is not always a rose" by any other name, especially when "someone whose name is changed to Montague or Capulet now may be perceived as someone quite different." (449). Allowing a person to choose their own name is especially important when a person's name may give some background into their 
cultural, political or ethnic background. Again, drawing on Lahman and colleagues (2015), the typical process of using anglicized pseudonyms to protect the participant's identity can alter the meaning and significance of a person's narrative. For this reason, rather than potentially strip a person of a key part of their identity, I wanted to give users the option to choose how they want to identify. They can offer their own name, choose to go by their nickname or choose a name they would like to be assigned or, finally, choose to be assigned a name at random.

Participants in my focus groups were not presented this option for three reasons: first and foremost, participants were not given the option to self-identify to protect those who might choose not to identify. As offering some participants the option to name themselves may give those named participants more ownership over their accounts than others, all names were removed. Secondly, as focus groups took place in person, I was able to build trust more easily. It was harder to so this with participants on the internet. Finally, removing names forces the reader to pay attention to the discussion, not who said it. In this case, as the questions were not biographical in nature, the risk of identification outweighed the benefit of allowing my participants the option of owning the data. Not wanting to assign pseudonyms and risk mischaracterizing my participants, I chose to number participants instead.

\section{Data Analysis Strategy: The Listening Guide}

To analyse both the focus group transcripts and user questionnaires, I adapted a method called the "listening guide" by Andrea Doucet and Natasha Mauthner. This method provides a "multi-layered" approach to the different dimensions of self-experienced by a respondent that can be gleaned by their response. Though not necessarily a conscious construction, the narratives established by participants through the choice of response constructs a particular social world reflective of, though not necessarily constituted by, respondent experiences (Flyvbjerg, 2001). 
This method of data analysis attends to the ways participants construct themselves in a web of relationships through their narrative or interlocution. Flyvbjerg (2001) notes that people who have achieved a level of competency in their lifestyle or vocation are unable to articulate the rules that govern their behaviour, because they have reached a level of competency that allows them to intuit cases in their experience. For this reason, simply reporting what is said does not necessarily shed light on the richness of a person's response. By attending to not only what is said, but how it is said, this guide is able to draw out a number of meanings from one particular narrative. It draws on various ways the respondent constructs themselves in articulating a point, to create a particularly rich tapestry of the social world they experience. This guide attends to the ontological, public, conceptual and metanarratives that frame the existence of respondents (Somers, 1994).

The listening guide that is used in this analysis draws on the works of Charles Taylor and Seyla Benhabib (Somers, 1994). Broadly, this perspective rests on the idea that the articulation of a point is simultaneously an articulation of the self (Benhabib, 1999). These articulations represent the set of relationships that shape a person's social world and enables their ability to act within that network. The analysis is accomplished by subjecting the interview transcripts to multiple successive readings, each attuned to a different aspect of the narrative. In the case of this analysis, there are four readings from which my data was generated.

\section{The Readings}

The first reading focuses on what is said by respondents. It establishes the themes and major events generated by respondents. The first reading is also a moment for the researcher to practice reflexivity by noting their experience of the data. This reading is accomplished by analysing the transcript twice simultaneously: first by listening to the interview and taking notes 
of events, themes, protagonists, conflicts, plots and creating a coherent narrative of my own from their responses. Second, I make notes of my own affective reactions to the interview data. In this stage of the analysis I treat myself as a participant in the generation of the data, noting my reactions to participants as well as their reactions to each other. This serves as both a tool for data generation and a check on my bias in interviewing and transcription ${ }^{5}$.

Given that HIV-risk and a person's sexuality are both especially personal and sensitive subjects, HIV prevention (or "safer sex") technologies will be shaped by the affective experience of the social groups with which it interacts. Because this project requires some attention to the affective experience of respondents, it is important to note moments when they distance themselves emotionally. The second reading attends to how the respondent positions themselves in their responses. Doucet and Mauthner (2008) note that this reading comprehends how person speaks about themselves and sheds light on both their perception of self the "parameters of their social world" (405). The second reading also attends to the shifting ontological status of my respondents as they shift from one state of animation to another throughout their responses. This reading draws on Sommers (1994) assertion that narrative location speaks to a person's argentic potential across space and time. It speaks to the shifting experience of self as a person moves from one set of relations to another. Further, As Chen (2012) notes the shifting of the subject

\footnotetext{
${ }^{5}$ It was key that I do this because, as a member of some of the communities that I am studying, I am also deeply invested in the norms, values and practices that ground these responses. It is worth noting that some users made me uncomfortable. Though I have tried to be charitable in my approach to analyzing their narratives, I have done so noting that some of these narratives "grossed me out" or where I wondered if the participant was overcompensating for behaviour that we both knew was "bad." It is also worth noting that listening to my participants, and reading the relevant social literature has changed my own perspective of the role of HIV in my moral code. So, while I thought some of the things my participants said were not to my taste, engaging with them fairly and openly has made me aware of a more complex set of relations than were available to me before.
} 
from active to passive, from proximal to distant, are indicative of a set of power relations that are built into a linguistically constituted hierarchy of being.

This reading is accomplished by tracing the shift of the subject in their narration.

Specifically, this entails tracing the 'movement' of the subject, whether that be from first person to second person, by speaking in collectives or generalizations or by omitting themselves entirely from the response. Examples include, when a person speaks about themselves in the eventful and commanding 'I' as opposed to the passive 'me,' who is done to; or when 'we' is employed to imply a sense of community or solidarity with a group. Attending to these moments can tell us about a person's social location, sense of agency and sense of self, without necessarily requiring the participant to articulate these points. This reading can help clarify a respondent's positon already articulated in reading one. Attending to these shifts, therefore, allow us to make the most of our respondent's responses and delve more deeply into the relationships that are forming around PrEP.

The third reading attends to the other 'characters' in a narrative who are not the narrator. This reading attends to the relationships respondents discuss, either prompted or unprompted, when discussing PrEP. It speaks to the immediate interpersonal relations and social networks between the respondent and the social groups they interact with, or imagine interacting with, when thinking about PrEP. This includes the audience with which the participant is interacting. Namely, myself and any other persons engaging with the narrative such as my supervisor, the other focus group members or other academics I address in presentations or publications. The third reading speaks to the fact that all interactions are necessarily relational, formed in relation to a human or non-human other in a network of established relationships that pre-existed that interaction. 
The final reading seeks to connect the micro-narratives of the respondents with macrolevel social processes. This reading attends to the metanarratives or conceptual narratives established by the respondents. This reading is not unlike the first reading, however, with a stronger emphasis on the established constructs of interest to sociologists. This reading represents the synergy of my expertise in sociology with the experiential experience of the respondent's concrete experience in the social world into which PrEP is infiltrating.

\section{The Analytic Power of the Listening Guide}

These readings shed light on a number of different relations, adding additional layers of interpretation to provide a more trustworthy account of a small number of responses. By subjecting the data to a number of lenses, the analysis more fully describes the social landscape and relations in which participants are enmeshed. By combining these analyses my project can make stronger claims than they would be on their own.

This analysis attends to both users and non-users' interpretation of PrEP in order to understand how these interpretations bear on the trajectory of PrEP as an intervention in HIV prevention. By examining the relations established between users, non-users and PrEP we can understand how they are co-constructed as PrEP is established as an intervention. As Jasanoff (2004) argues, "the way we know and represent the world is inseparable from the way we choose to live in it" (2). In other words, the material embodiments of knowledge, personal know-how and knowledge itself are co-constitutive with social life (3). Attending to both groups' experience of PrEP as a semi-malleable, semi-obdurate actant is necessary for establishing impact of a technology on a community.

By taking into account the narratives of both users and non-users as social groups relevant in the implementation of PrEP, this analysis can better understand how PrEP stands to change or be changed by the social groups in which it is acting. This analysis strategy is 
particularly useful as it attends to the social relationships as they related to the material reality of everyday lives of respondents. Further, this strategy foregrounds both the social and material relationships that lead to the crystallization of a particular technology. It attends to the affective experience of respondents as they interact in that ensemble.

In addition to this method's attention to the layers of relationships that are discussed by respondents, this method has a built-in member checking mechanism. This member checking mechanism enhances the validity of the data. By interviewing users and holding focus groups with non-users we can understand the fractures between and within these two groups. We can also test ideas as they are brought up in discussion. By routinely asking whether or not an idea resonates with the group we can establish which points are reflective of strong social currents and which ones represent those which are less pervasive. As we will see, there are moments when the position articulated is complicated by contradicting experiences voiced by another participant. The use of focus groups, as I have noted, is important for articulating the shared or diverse understandings of an object (Morgan, 1997, p.15). This method creates a situation that requires interested parties to form and articulate a position that is then either taken up or discredited by the group. In this way focus groups are useful methods that have built in methods of member checking that helps ensure the quality and trustworthiness of my findings (SchwartzShea \& Yannow, 2012).

Further, this method requires the researcher to engage in two reflexive readings of the material, once as it is being generated as a participant facilitator, and again in the first reading as part of the thematic discussion. Doing the first reflexive reading allows the researcher to understand their own characteristics, personal biases and social location as they generate data (Schwartz-Shea \& Yannow, 2012 p.100). These readings provide for an honest discussion of the 
material than one that purports the information was generated independently of the researcher and that the information data somehow speaks for itself. These readings, in addition to memberchecking serve to produce particularly rich sociological data.

\section{What Cannot be Known}

While this guide is useful for a particular type of analysis, it is important to note exactly what this analysis cannot do. First, this analysis does to answer questions that rely on large numbers to generate trustworthy data. Though I discuss relations that exist on a meso- or macro scale, this is not done through the lens of individual experiences of these large-scale processes or relations. These experiences are not necessarily generalizable to entire populations. As such, questions that purport to explain or predict a hypothesis are not compatible with this type of data analysis. Rather, this analysis speaks to the dynamic nature of an assemblage made up of interwoven and heterogeneous actors (Rosengarten \& Michael, 2010).

My selection method is also not generalizable in the positivistic sense. Participants were not randomly selected nor were they selected on the basis of any population quality, other than the fact that they were either users or non-users in a particular location. For that reason, this data may not generalize to the entire "population" of men who have sex with men. This was done deliberately, however, as this is advantageous to studying the use of PrEP by broader queer persons in such a way that avoids treating all MSMs as a singular risk category, an approach that has been found problematic (Young \& Meyer, 2005). Rather, participants were allowed to selfselect, assuming that persons who wanted to discuss PrEP were interested in PrEP because they had something of substance to contribute. Doucet and Mauthner's (2008) listening guide is employed understanding that this analysis is intended to understand relationships as opposed to the "reality" of the narrator's experiences. This method, therefore allows us to understand the 
relationships between users, nonusers and PrEP in such a way that is more complex than those in the health literature, who rely on identity or behavioural categories to conduct their analysis.

While the respondents are trustworthy as "knowing but experiencing subjects" (Stanley, 1993), this analysis does not attempt to know the participant "below" the narrative (Doucet \& Mauthner, 2008). Rather, it attends to the relationships in which that person is enmeshed. In the words of Plummer (1995, in Doucet \& Mauthner, 2008), it is everything except the lived lives of the participants, "it speaks all around the life: it provides routes into a life, lays down maps for lives to follow, suggests links between life and a culture...but is not the life" (404). It is important not to confuse the themes identified and discussed in the following chapters with the "actual" lived experience of my respondents. What I am reporting is not an "objective snapshot" of their lives, but a map of the social world that is informed by their contextual and expert experience redrawn through the lens of sociological inquiry.

What is provided by this analysis is then a map. This map describes a social landscape that is replete with blocked paths, fields of inquiry and streams and currents of power. Though these images, sometimes crudely drawn, represent avoidable hazards and promises safe passage on paper, a map does not purport to be the landscape it represents. Depending on the author, some landmarks are foregrounded and features exaggerated. Yet the level of detail or the likeness of the map to the world it represents of does not stop the traveller from using that map, knowing that the person who has provided that map, has foregrounded important landmarks and omitted details irrelevant to the navigation of that landscape. This is the nature of communicating phronetic knowledge. It is necessarily incomplete and targeted to a particular social landscape. Much like a map, the use of phronetic knowledge is to answer value-rational questions such as "Where are we going," "Is it desirable" and "What should be done?" (Flyvbjerg, 2001, p.6). 


\section{Chapter 4 (Mis)configuring the User: Barriers to Access and the Configuration of the PrEP User as "At Risk"}

As PrEP has only become available in the last year, it is not entirely clear how, and for what reasons, persons use it. Though PrEP is indicated to be used only by those at "substantial" risk of contracting HIV, according to a recent study by the Centers for Disease Control, over half of gay men sampled indicate they would like to use PrEP (Hoots et al., 2016). Far more than those realistically at risk of contracting HIV. At the same time, the report noted that PrEP take up continues to be low among queer men, hovering around 4\% (Snowden et al., 2016; Hoots et al., 2016). These irregularities point to a potential disconnect between interpretation, access and use of PrEP.

According to Wyatt (2003) non-users can be subdivided into two groups: The "have nots," those who want to use a technology but are unable for some reason and the "want nots," those who resist or reject a technology voluntarily. This chapter examines the barriers to use that either expel or exclude would be users from taking the drug. Though it is certainly the case that users and non-users are actively resisting PrEP as it is currently defined, recent studies on PrEP uptake seems to indicate that the proportion of persons who want to use PrEP drastically outweighs those who access the treatment (Hoots, 2016; Snowden et al., 2016). For this reason, there is strong evidence that persons who are not using PrEP are not just resisting use but that barriers exclude or expel interested parties from the category of "PrEP user". Indeed, both users and non-users indicate that barriers to use exist throughout the process of becoming a PrEP user from understanding one's self as at risk to convincing their doctor that they are at risk, to paying for the treatment. They also note that stigma both on the side of doctors and among their peers 
could eventually expel them from the user category even once they managed to secure a prescription. This stigma, I argue is perhaps the most pervasive and deep-seated barrier to PrEP's accessibility. Finally, I note that some participants explained the side effects, especially the chance for weight gain that comes with some anti-retroviral treatments may cause them to stop treatment. In the following chapters, we will examine how and why users and non-users reject or resist PrEP as it is currently designed.

\section{Barriers to Use Identified in the Literature}

Academics in medicine, medical ethics and the sociology of health have all hotly debated how PrEP might be used and which barriers might render it less effective. Broadly, these academics have offered a number barriers such as the possibility that HIV will mutate a resistance against PrEP, rendering Truvada ineffective as both a prophylactic and as a treatment. There is also concern that persons would not be able to keep up with the one-a-day dose and/or will become overly optimistic about their new-found HIV "immunity," both leading to a potential for increased HIV transmission (Desai et al., 2015; Beaten \& Grant, 2013; Rowniak \& Portillo, 2013; Underhill \& Meyer, 2013). Others note that the drug is currently plastic, as it is being reinvented along the lines of dosage, dosing frequency and the parameters of treatment scheduling (Beaten \& Grant, 2013). Further, the definition of who is "at risk" is also a theme that bears on the use of PrEP (Beaten \& Grant, 2013; Rowniak \& Portillo, 2013).

Though these barriers are key to understanding PrEP's role in HIV prevention, they fail to fully comprehend the role of PrEP in the relationships that it impacts. Science and technology studies provides a number of examples that demonstrate the importance of the role of the social in the development and ultimate success of a technology. The most significant insight for this discussion is that success of a technology largely depends on whether or not its inscribed use 
corresponds with the needs of the intended user group (Woolgar, 1991; van Oost, 2003, Pinch \& Bjiker, 1984; Wyatt, 2003; Akrich, 1992). Martin Holt (2015) explains that PrEP, like any other technology, is configured with a particular user in mind. At the design and testing stage, the technology is configured with an "ideal user" in mind. This ideal user is any man who has sex with men who is at a high risk of contracting HIV. It is certainly the case that PrEP is scripted with a particular user in mind, one must simply look to the product pamphlet for an explicit list of recommended user groups. The ways that members of the HIV-assemblage either fail to correspond to this definition (or actively resist the script) indicates whether or not the drug will become a successful HIV intervention or whether it might change (for a discussion of the flexibility of the pill in design see Rosengarten \& Michael, 2009a/b).

In order to be considered an appropriate PrEP user, a person needs to be categorized as "high risk" for HIV transmission. Gilead Sciences $(2013,2016)$ breaks down "high-risk" for an MSM in this way: They must engage in sexual activity in a network with a high prevalence of HIV, or have an HIV-positive partner and must either: have a history of inconsistent or no condom use, have been diagnosed with an STI, engage in sex work, use illicit drugs or have an alcohol dependence and/or be incarcerated. A person may also qualify for PrEP if you have a partner of unknown HIV status who fits any of these criteria. This definition is incredibly broad, as networks of HIV prevalence can be interpreted in a number of ways and STIs are increasingly common in Ontario (Public Health Ontario, 2017, 2016, 2015). However, as we will see, the inscribed user does not necessarily correspond with the effective PrEP user. Holt (2015) notes that in addition to these constraints, there are a number of other qualities necessary for successful use that are not outlined in this definition of an ideal user as being cautious, compliant and organized (and not much else.) The partial configuration of this technology allows for some 
flexibility between the ideal user and the effective use of PrEP. In order to understand PrEP's chance of success as an HIV prevention drug, it is necessary see how the effective PrEP user matches up with the inscribed user.

The current configuration of PrEP as a technology calls for particular characteristics from its users. Holt (2015) has studied the narratives constructing the effective user and has defined the effective PrEP user is configured along the following lines. Ideally a PrEP user should be able to keep up with their regular dosing (a finding that Holt notes it not exclusive to PrEP). Evidence from Holt's (2015) study suggests that the effective PrEP user is also required to be discreet, as the pill and its use carries a variety of meanings across social groups. This finding is echoed by Young, Flowers and McDaid (2016) who found that PrEP could be mistaken for an HIV treatment and consequently could be misinterpreted as evidence that the user hiding their HIV-positive status. Holt (2015) notes that the effective PrEP user ought to be honest and aware of their HIV risk and the limitations of their sexual practices as well as possess an awareness and trust in the medical community.

In discussing their experiences and perceptions of PrEP, my participants identified a number of barriers that force them, or users like them out of the effective user frame. Though many users and non-users alike fit the criteria of an ideal user, and indeed envision themselves as users, they identify a number of difficulties that have to be overcome in order to become a user. Users discussed a number of barriers associated with the process of successfully becoming a user, though they identified far fewer barriers than the non-user group. The most salient barrier to PrEP users was convincing their health care provider to prescribe PrEP and, to a lesser extent, difficulty funding their treatment. For non-users, however, the cost of treatment was far more of a concern. 


\section{Barrier: The cost of treatment}

Among one of the most obvious barriers to PrEP use identified both in the literature, in the media (CBC News, 2015; Smith-Cross, 2016; Strapagiel, 2016; Weeks, 2015) and in LGBT media (Miksche, 2017; Bell, 2016) is that the sheer cost of PrEP is a substantial barrier to most potential users. Though estimates vary, PrEP treatment in Ontario seems to hover around $\$ 1000$ a month. This is certainly a substantial barrier for any potential PrEP user who is not insured or independently wealthy ${ }^{6}$. This has led to some argument that PrEP may be a drug exclusively for the socially privileged (Speildenner, 2016; Poicus, 2016). The expense of this treatment, and the restricted access to PrEP to only the socially privileged was certainly apparent from my respondent's discussions. Income and financial independence was certainly an issue for persons who were not able to pay for PrEP out of pocket. For a number of users, the cost of treatment did not come up. Though I am wary of drawing any causal connection, it is worth noting that those who did not mention cost were gainfully employed with an income of over $\$ 80,000$. Users who discussed cost were either transitioning from gainful employment to a more precarious financial positon or employed with a lower income.

Although the cost of the treatment did not come up as an especially pressing theme, Mark, Eric, Richie and James all discussed how the cost of the treatment affected their lives. Though not necessarily a barrier for himself, Mark notes that in discussing PrEP with his peers, he has come to realise that most persons in his social group simply cannot afford PrEP, despite

\footnotetext{
${ }^{6} \mathrm{PrEP}$ is covered by the Trillium drug benefit program for HIV treatment. Some people are using a loophole in the program to access Truvada for PrEP. None of my focus group participants indicated they were using this plan or that they were aware of using it to access PrEP. Further, it is unclear how the Trillium benefit program may impact PrEP use for students or persons who are living semi-independently from their families, even if it was approved for prevention.
} 
being interested in taking the drug. This points to an uneven deployment of PrEP in the queer community so long as PrEP remains unsubsidized. Though some participants accessed PrEP with relative ease, for others it was significantly more challenging, in part due to the cost of the treatment.

Eric, James and Richie elucidate the barriers for persons who cannot afford the drug independently. Each of these participants accessed PrEP through their insurance plans. Eric, for example, discusses two ways he has accessed PrEP. He notes that he has recently changed his career path, which has resulted in a change in his ability to access PrEP. When Eric left his job as a veterinarian (where he had full coverage under a drug plan) to become a graduate student, it was necessary for him to end his treatment. Eric has considered starting his treatment again under his university drug plan. If he were to do so, however, he would have to fund a portion of his treatment to the tune of between 80 and 120 dollars a month. Eric writes that he imagines this cost would be unsustainable for any student (something seemingly confirmed by my non-user participants). James, however, is a graduate student, who notes that he is covered " $100 \%$ " between his parents and his student health plan.

Richie was the only user participant who was explicit about struggling to pay for PrEP. He was not able to pay out of pocket and was concerned that to access PrEP through his work would only partially cover his treatment. Fortunately for Richie, he was insured in totality. But this was not the only problem. Though insured, he was nonetheless worried about disclosing his PrEP user status to his employer. Richie was concerned that starting PrEP would raise premiums for the small company for which he worked, or that he would be "questioned about how much insurance [he] was using." These questions would inevitably force him to reveal himself as a 
PrEP user, drawing undue attention to his personal life. Even if he was fully insured, he was not convinced that his health plan was confidential, which kept him from accessing PrEP for months. Richie's story indicates that some users experienced financial barriers that might have stopped them from becoming a user, overall financial independence and cost was a more significant issue for non-users. A minor theme for my user respondents, the cost of the drug was clearly a substantial deterrent for non-users. Like users, both focus groups were entirely willing to take PrEP, if it was available to them and within their means. However, most non-users expressed concerns about the price and their ability to afford PrEP. Both focus groups identified that the treatment was beyond their means without significant support. For example, learning about the cost of the treatment causes 009 to immediately re-evaluate his identity as a potential user. 007 , on the other hand was optimistic, stating that he will pursue treatment as soon as Ontario covers the treatment. Both focus groups were aware of the financial burden of PrEP treatment and identified a number of ways they might offset the cost, including relying on their parents' insurance. However, the relationship between PrEP and financial dependence elucidates an important contradiction that may exclude a large number of younger would be users.

The cost of the drug and its relationship to their independence from parents was of particular concern to members of the first of my two non-user focus groups. Participants in this group noted that they could potentially pay for the treatment though their parents insurance plan, however, they were unwilling to do so as they did not want their parents to know they were taking PrEP. Unlike James, this participant (002) noted that he is not interested discussing PrEP with his parents or even risk them discovering he was taking PrEP at all. Even though he and his parents have a great relationship, 002 keeps his sex life private from his parents. He says he is not interested in approaching his parents to ask, "Hi, can I get a drug that will help me have safer 
sex?" Responding to 002 's point, participant 001 notes that even though they could get treatment through their parent's insurance plans, being in a relationship with a person who is HIV-positive is stigmatized and it is particularly difficult admitting to a family member that you might be having sex with a person who is HIV-positive. Effectively, 002 has to weigh his risk of contracting HIV with the risk of losing face with his parents.

Pressing 002 further, I asked if his qualms discussing PrEP with his parents stems from a discomfort around sharing one's sex life with family. I ask if buying PrEP was similar to buying a sex toy online, for example. Presumably, if he was only worried about keeping his sex life private, he would confirm that PrEP and sex toys are similar in this regard. This is not the case, as 002 explains. In fact, to my surprise, 002 is not at all worried about whether or not his parents discovered he had bought a sex toy. Rather, he is concerned that as PrEP is used to prevent someone from becoming HIV-positive, he would cause his parents to worry about whether or not he was safe. He feels discovering he was a user would bring unwanted attention to his sexual practices; potentially exposing himself as a user and prompting uncomfortable conversations about his HIV risk with his parents. He does not want to worry his parents or imply that he is getting into "unsafe sexual situations." If he was insured independently from his parents he would be much more likely to start using PrEP. Two users ${ }^{7}$ also noted that they have not told their parents that they are on PrEP. Evidently, the stigma associated with HIV risk requires the effective PrEP user to manage their PrEP user status around family members.

In the event that the benefits of reducing HIV risk are outweighed by the harm it might cause to their relationships it is understandable that some people may decide to stop pursuing

\footnotetext{
${ }^{7}$ I have chosen not to identify which users have indicated this, as it may inadvertently identify them to those family members.
} 
PrEP simply to save face. This is especially apparent for young MSM. The relationship between familial dependence and PrEP use seems to represent a significant barrier for younger or less independent PrEP users. Participant 001, for example, explained that if his father discovered he was on PrEP it would most certainly spell the "death" of his social life. Additionally, 001 notes that he would not feel comfortable speaking to doctors about PrEP, either. This is not exclusive to PrEP, however, 001 is uncomfortable meeting his family doctor to address any aspect of his sexual health. Rather than face the "man who weighed [him] as a baby", 001 visits anonymous clinics around Ottawa where he feels more secure in his anonymity. The room nodded in agreement to this point. It is unclear whether they themselves have experienced this, or if they were only sympathetic.

Keogh (2008) notes that HIV-negative gay men who are not in regular contact with HIVpositive persons understand HIV as a far more significant problem than those in regular contact with HIV-positive persons. Regular contact with HIV-positive persons as associated with a decreased sense of stigma around HIV. Similarly, in a study of HIV-negative black college students, Payton and Kvasny (2016) found that in addition to the stigma of being infected with HIV there was a stigma of simply participating in HIV prevention discourse. Dubbed "stigma by association" by Payton and Kvasny, this stigma was a salient barrier in black college students desire to access HIV prevention materials. Despite being aware of their risk status, black college students in this study were reluctant to simply investigate HIV prevention. Payton and Kvasny's participants were afraid of being publically associated with a stigmatized disease, even if they were taking proactive steps to prevent contracting the disease. In this study, the participants engaged in some impression management to avoid the association with the disease. Similarly, Young, Flowers and McDaid (2016) found that one of their participants managed stigma by 
"distancing herself from HIV and limiting the possibilities of exposure to this stigma." (419) Though the nature of HIV-stigma is certainly not the exact same for black women as it is for the predominantly white queer men who populate this study, participants in both the user and nonuser group alike point to the existence of this phenomenon in their own access to HIV prevention materials. This stigma remained an issue that extended into their access to PrEP. As it is made clear by 002, 001 and Richie's comments, simply being associated with HIV is enough of a concern to turn a user into a non-user.

While being associated with HIV risk is certainly a concern for many of my participants, it is unclear how pervasive this barrier might be - as participant 009 in focus group 2 noted that his parents would likely be the first persons he talked to about PrEP once he started treatment. He also shares a family doctor with his mother, and spoke fairly lightly of the process, stating that he would likely tell her first, as a "heads up," as they share a pharmacist and she has permission to pick up his medications. Likewise, as outlined above, James accesses PrEP through a combination of his student and parental health plan. It seems that this barrier does not exist for all potential PrEP users who are dependent on another person for their medical care.

Regardless, it appears that if PrEP is to be fully accessible to those who want it, avenues for PrEP to be accessed confidentially must be created. Confidentiality remains a serious concern for adolescents wanting to access health care (Klein et al.,1999). This was found to be especially true for adolescents who engage in perceived high-risk behaviour such as condomless sex, smoking and alcohol use (Leherer et al., 2007). However, these persons are among the persons who might benefit from accessing healthcare the most (ibid). Richie and 002 underscores exactly how would be users might be dissuaded from accessing PrEP due to the undesirable connotations about those accessing PrEP. For PrEP users who are reluctant to label themselves at risk, 
accessing PrEP through their insurance may be impossible. Despite the fundamental commitment medical practitioners have in maintaining confidentiality, these guarantees are at odds with the current processes of compensation or with the insurance system itself. The Guttmacher Institute (Gold, 2009) notes that the current health insurance system allows for substantial confidentiality breaches. They note that, in addition to half of university students who are insured under their parent's plan, “virtually all adolescents... are covered under a parent's policy” (13). Therefore, if PrEP is to become a successful HIV intervention that is accessible to anyone at risk of contracting HIV, PrEP must either be reconfigured for everyone (as opposed to only being available to members of a vulnerable population at substantial risk of acquiring HIV) or made available outside the insurance system, where it can be accessed anonymously.

\section{Barrier: Access to Sympathetic and Knowledgeable Health Care Practitioners}

While non-users were certainly more concerned about the process of acquiring and securing the means to sustain their PrEP regimen, users indicated that convincing their health practitioner that they were an ideal user was perhaps the most significant barrier. The most salient barrier discussed by users were attempts made by doctors to dissuade them from undertaking the treatment. It is clear from the high variation in responses between users on this subject, that the process was not particularly straightforward. Users were often referred to specialists or required to make several appointments with their doctor to successfully become a PrEP user. Though users spoke positively of their experience in other ways, their experiences were generally unpleasant ordeals. Eric was the sole participant who discusses the majority of this process as non-judgmental and an overall enriching experience.

Eric's experience of engaging with the health care system is notable in several ways. Contrary to other users, he was not actively searching to take PrEP, but was actively encouraged 
by medical professionals to become a user. Following an STI diagnosis, Eric was referred to a clinic specializing in immunodeficiency in order to discuss PrEP. Though Eric felt compelled to go, he was happy to go to learn more about the drug. He notes that he felt comfortable discussing medicine as he has a medical background and welcomed the opportunity to discuss a drug relevant to his sexuality. Due to his training as a veterinarian, he notes that he "trusts and understands" the health care system. He was however, taken aback by the fact that the health clinic that referred him to the immunodeficiency clinic clearly identified him as an ideal user. He notes that the nurse at the health clinic told him they knew the "type of person" who gets HIV and that he "looks like one of them." His experience at the immunodeficiency clinic was far more pleasant. He describes the clinic as "warm and supportive and not at all judgmental," in stark opposition to the "degrading processes" he experienced at other sexual health clinics. Eric notes in contrast to being framed as an ideal user at the other clinic, he felt no pressure to become a user. He had an open and frank discussion with another medical practitioner about the "pros and cons" of taking PrEP. He notes that even though the practitioner was straight, he felt no judgement.

Vince also had a relatively straightforward experience, though he spoke far less about this part of the process than Eric. Vince notes that when he went to start his treatment, at the time his doctor was unaware about PrEP, but was open to prescribing it. The doctor did a bit of research and advised Vince on the side effects. At that point Vince left the office to further consider whether or not he should take PrEP. A few months later he had a "bit of a scare after learning the truth about one of [his] sexual partners." This scare finally convinced him to meet again with his doctor and take PrEP. This second time around the doctor was far more aware of PrEP. In fact, 
Vince writes, that a number patients had brought PrEP up after his visit. Now that the doctor was far more familiar with PrEP he now felt far more comfortable prescribing it.

Vince and Eric's experience are rather different from their peers, many of whom seemed to have difficulty framing themselves as users. Where Vince encountered little resistance compared to his peers, others encountered reluctant or outright hostile doctors. Doctors generally seem to dismiss users or at least try to dissuade them from becoming a user. James, Christian and Marc all had a difficult time convincing their practitioner to start treatment. It is perhaps the case that this is due to doctors being unaware of what constitutes a PrEP regimen or perhaps that Truvada has been long indicated for HIV treatment. McMullen (2006) explains that when it is the case that patients are misinformed or appear to know more than their practitioner, there is a possibility that the practitioner will dismiss the patient as either unknowledgeable or ask them to return once they have researched the treatment further. It appears that for participants who had a good relationship with their doctor or some background in medicine, acquiring PrEP was not as grueling as for others.

When doctors are less knowledgeable about a treatment than the patient, McMullen (2006) argues, the doctor can either trust the patient at their word, ask the patient to comeback with more research on the topic while they learn more about the treatment in the meantime or they will simply deny the patient the treatment. In Mark's case, it appears no amount of preparation would convince his doctor. Mark's family doctor would simply not prescribe him PrEP as unconvinced that PrEP could live up to its reputation. She "thought it was too good to be true." Even when he brought CDC fact sheets and multiple peer-reviewed studies, she remained adamant not to begin treatment. Rather, she referred Mark to a specialist. He found the process to be "super annoying" and notes that this alone would likely cause many people to give up. In 
contrast to his family doctor, the specialist, who Mark noted was gay, was more aware of PrEP, was quite encouraging and "prescribed it right away." Mark finishes his point on this subject by noting that his family doctor still will not take over his treatment, meaning that he has to visit another doctor in order to continue treatment. Both Mark and Christian experienced this sort of "judgement" at the doctor's office. Whether it was because the doctor was concerned about the user's intentions with respect to PrEP or a reluctance to offer a drug they were unfamiliar with, participants fought an uphill battle to convince their doctors that they were not simply “irresponsible," but in fact high risk and in need of PrEP.

In contrast to Mark, who was looking for some security in his sex life, Christian notes that he pursued PrEP originally because he was in a serodiscordant relationship and wanted to have an "extra layer of protection," presumably should a condom fail. Unfortunately for Christian, this was not enough of a reason for the doctor to prescribe him PrEP. The doctor remained unconvinced that the treatment would be beneficial and spent a half-hour "lecturing" Christian about the risks of taking PrEP, ultimately suggesting he stick to using condoms. Though the doctor acknowledged that condoms are not completely effective either, they continued to pressure Christian to abandon his attempt at becoming a user. Christian noticed that the doctor had a "moralistic tone" when arguing with him about PrEP which made Christian feel "unsure" about whether or not he should be taking PrEP.

In this instance, Christian may have been successfully dissociated from the ideal user category, should he have not formed a bond with other PrEP users. This speaks to the importance of community, in this case the community organizing around PrEP, to reframe PrEP use from "selfish" desire to go without condoms with his HIV-positive partner to being part of a larger HIV prevention initiative. It appears from Christian's writing, that simply trying to protect 
himself from infection was not enough for him to become a user. It was only after joining a community of PrEP users and reading a particular inspiring article on PrEP use that he felt empowered to convince his doctor. He writes:

It wasn't just a selfish decision to allow myself to indulge in condomless sex; I wanted to be a trailblazer and show that PrEP worked and that with this technique (medication), we could stop new infections. It could act as a stop gap solution until a cure to HIV could be found. I was willing to experiment with my body and take the risk, for a good cause.

Christian writes that later that month he visited his doctor again. Noticing that this time he was determined to become a user, the doctor "put up less of a fight" and began the treatment prescreening. If it was not for the fact that his friends offline were increasingly seroconverting, and the fact that he was reaching out increasingly to other PrEP users online, Christian imagines he may not have successfully become a user.

Finally, James' experience points to problems arising from the scarcity of doctors that continues to make adequate healthcare a problem for many Canadians. As PrEP requires regular contact with a doctor at least every three months, it is understandably difficult for transient users to continue treatment. James's partner had an easier time getting PrEP than James himself. According to James, his partner has an excellent relationship with his doctor. His partner has had the same doctor since he was born, with whom he has always been open and honest about his sexuality. The doctor, despite being unfamiliar with PrEP simply "googled it and wrote his prescription." James experience on the other hand, was more difficult. As he had recently moved to Western Ontario to study, he found it rather difficult to find a doctor who was taking patients in his region, let alone one that was LGBT friendly. After a few weeks of searching, James found a doctor taking patients in a nearby town. Unfortunately, the doctor was not particularly open minded. James writes how he was "casually slut shamed for having an open relationship and judged for recreational drug use" at his first appointment. After two months of bouncing between 
doctors, he found a PrEP friendly doctor at his current university health services. This doctor was far more cooperative. After some research and confirmation that James was indeed in the "high risk" category, James went on to get some bloodwork done and was prescribed PrEP within a week.

Focus group members were a bit less vocal about their concerns with respect to doctors. Likely because they were not asked questions about doctors directly, while users were asked to discuss how they managed to get a prescription. Though what little they did mention is important. For example, 003 notes that he is acutely aware of the difficulties he has had accessing healthcare for other treatments in the past. He noticed that in his experience doctors are often "poorly informed" or "have stigmas." 003 explains that, from his experience with this sort of doctors he imagines that interactions between doctors and patients trying to take PrEP will be an uphill battle. Indeed, it seems that like the users above, 003 is aware that some doctors might disapprove of the lifestyle associated with PrEP usership. Whether or not this is because the doctor disapproves of homosexuality outright, or simply that they are concerned about disinhibition. Thus, it is not just accessing knowledgeable doctors, but ones that are nonjudgmental and sympathetic to the realities of some communities that would likely benefit from PrEP that will ensure that PrEP becomes a successful intervention. In order for this to happen, it is likely that family doctors will need to become familiar with the benefits of PrEP use, despite their discomfort with the lifestyles of some users.

Non-users were more preoccupied with how to negotiate their desire to use PrEP with their risk profile. On one hand, some participants noted that they would not be able to convince their doctor that they were high enough risk for PrEP, others were concerned that divulging their actual risk profile to the doctor might damage their reputation with their doctor. Participants in 
both focus groups made it clear that they did not think they would be able to successfully negotiate these two barriers.

It becomes clear from the discussion in the second focus group that a number of nonusers imagine they would struggle to convince their practitioners they meet the current criteria for PrEP use. Their behaviour, motivations to take PrEP and the prescription criteria do not match. 009 notes that, despite being very interested in the drug, he is dissuaded by the "long list of criteria" that he has to meet to be approved for PrEP. I note that he would only have to meet one of those criteria to become a user. Nonetheless, he responds, if he does not meet any of those criteria, he cannot be considered. He wants to take PrEP as a precautionary measure, not necessarily because he is a "high risk" as characterized by the list provided by Gilead. I point out that some of the criteria remains open to interpretation, such as "inconsistent condom use," that a person could use to convince a doctor that they are a "higher risk" than they might be in reality. However, I agree that this is an easy way to get written out of the PrEP user group. I ask if this discussion resonates with the group, to which 007 groans out a 'yes.'

Non-users also pointed out that the process would require them to discuss parts of themselves to their practitioners that they were not interested in sharing, in order to receive treatment. This barrier, that a potential PrEP user would need to discuss aspects of their lives that made them uncomfortable, embarrassed or they felt cast them in a negative light. In addition to the concerns outlined in the previous section, 001 lists a number of apprehensions about the process of becoming a PrEP user that would ultimately stop him from gaining access to treatment. While he is excited about PrEP and sees how it would be useful in his life, he is aware that it is inaccessible for a number of reasons. Firstly, as outlined above, he does not have regular access to a doctor with whom he feels comfortable discussing PrEP. We know from the 
experience of users, that this regular access likely filters out a number of potential users.

However, a number of users persevered and secured a doctor exclusively in order to sustain their PrEP use.

001, however, points to a more difficult barrier to overcome. Because he is transgender and a sex-worker he feels he is in an "awkward positon" that keeps him from getting access to PrEP. Though he meets multiple diagnostic criteria, he sees himself as likely unable to secure a treatment. He does not want to discuss his sex work with a doctor because of the stigma around sex work, though this would likely guarantee him access to the user category. In order to avoid being labelled a sex worker by his doctors, 001 notes that he would rather discuss PrEP as an MSM. However, his reality as a transgender man makes that route to starting treatment unlikely as he notes his doctors rarely recognize him as a man. Because he does not "pass as a man" nor has he began his transition he feels he will be written out of the process entirely because he has a vagina. He notes that doctors in general are not likely to see transmen as gay men and therefore would not be able to access PrEP, without giving up his treatment for "people with uteruses." Whether or not transmen can access PrEP as part of the MSM community is not entirely clear as remains it is up to the doctor to decide if "persons with uteruses" are at the same risk as other MSM whose risk is far more clearly established and are explicitly mentioned by Gilead. ${ }^{8}$

These narratives point to a wide array of inconsistencies and points to the interpretive flexibility of both the drug and the user group. Mol (2002) notes that illness has to be performed in a very particular way for it to be intelligible to a specific practitioner. HIV risk here can be

\footnotetext{
${ }^{8}$ Also, according to Hull, Edward \& Kelley (2016), Truvada takes longer to reach concentrations necessary to be effective in transmen than in cismen and transwomen. For this reason, other considerations need to be taken up by health care practitioners offering care to transmen. Though this thesis does not aim address the experiences of transmen specifically, it is evidently the case that PrEP is not well defined for the LGBT* community.
} 
performed in a number of ways, as can the categories of HIV risk including the gendered performance required to convince a doctor that one is rightly an 'MSM'. Though Gilead offers a number of very clear and reasonably broad diagnostic devices one may use to construct a user, it remains in the realm of the practitioner to interpret those diagnostic devices in light of the performance of that risk category. This is further complicated by the level of expertise and experience the practitioner has with HIV and novel HIV prevention technologies.

McKay (2011) identifies a perception of a lack of sensitivity on the part of health providers as the "[t]he most significant health disparity among LGBT people," as it results in the avoidance of routine health care and, for our purposes, reduces access to PrEP. The perception that doctors are hostile or judgmental of persons seeking HIV prevention is compounded by the novelty and community based nature of this drug. One key difference between PrEP users and a number of other patients is the influence of community on their decision to take PrEP. Many PrEP users have to educate their doctors. The novelty of Truvada as PrEP and the user to user information sharing creates a significant challenge to doctor's medical authority. McMullan (2006) notes that there are three possible outcomes that arise from the interaction between an internet informed patient and their practitioner. In some cases, uniformed or less technologically literate practitioners may feel their medical authority being threatened by the patient and will react defensively or dismissively to the information presented by the patient. Other cases, practitioners request the patient do more research on the subject, offering them what McMullan refers to as "internet prescription" (27) to reliable and accurate information. In the final scenario, the practitioner collaborates with the internet informed patient, allowing the patient some control over their medical care and sharing the burden of learning about the condition they are treating. 
Each of these possible scenarios occur in the narratives of users, however, in addition to being dismissed as "unknowledgeable," users routinely interpret this dismissal as either homophobic or judgmental. In their 2012 study anticipating challenges to PrEP use, Galindo et al. note that this was a significant concern for their participants as well. They write that "mistrust with medical settings still exists within communities impacted by HIV, and even those with access to providers expressed concern of homophobia and non-culturally competent staff in the medical field." Echoing these findings Galea et al. (2011) note that participants in their study indicated that they were concerned about the lack of sensitivity on the side of healthcare practitioners and a fear of discrimination with respect to their PrEP use.

\section{Barrier: Stigma, the Disinhibition Hypothesis and the \#TruvadaWhore}

The barriers above all stem from the characterization of PrEP users as either irresponsible or vulnerable. Rather than characterize PrEP users as responsible sexual actors, PrEP has been indicated "for those at the highest risk for HIV," to be used with condoms. However, this mischaracterizes the PrEP user, who are at risk specifically because they do not use condoms and requires the user to bear the stigma of being "at risk." Whether "irresponsible" or "vulnerable," either one of these frames serves to alienate potential users and non-users alike from using the drug and from communities of non-users who have interpreted high risk as promiscuity. Thus, perhaps the most significant obstacle to PrEP as a successful intervention is the implicit stigma built into its current indication for use.

Because PrEP has been framed as a drug for persons at high risk or a drug that allows a person to circumvent established traditions of sexual hygiene, such as monogamy and condom use, it has become a highly controversial drug in both the medical community and in the gay community itself (Belluz, 2014). Speildenner (2016) notes that health care providers continue to 
be reluctant to provide PrEP, in part due to concerns about cost and fears of the messaging around PrEP, in addition to the concern that PrEP will undermine other HIV prevention techniques. For example, Dennin (2016) notes that there is precedent in the history of HIV treatment and prevention, where the invention of effective treatment options, in particular highly active antiretroviral therapy (HAART) in the 1990s, resulted in a "movement towards a subculture of increasing risky sexual behaviour in the at-risk populations" (159). This 'disinhibition paradox hypothesis' is the sense that the large-scale implementation of PrEP would reduce the "behavioural constraints for men who desire condomless sex" (Young, Flowers \& McDaid, 2016, p. 414) which results in an HIV prevention technology that paradoxically increases the risk of HIV transmission. This hypothesis is not only skewed in such a way that it might misunderstand the way MSM identify themselves as users, but it appears to be internalized in such a way that it causes substantial emotional distress in those who see themselves at risk.

The disinhibition hypothesis assumes that those applying for PrEP are more or less homogeneous in their identity as users. That is to say they represent a group of users that are homogeneous in their values, such that they can use PrEP to engage intentionally in otherwise harmful behaviour. Dennin (2016) makes note that the reason to be concerned about PrEP is that adopters belong to a subculture within risk groups who adhere to "an alternate set of own norms which are incompatible with the tenets associated with the responsive and ethical standards of civilization" (161). While the arguments made in this paper are highly questionable, as would be PrEP users do seem to heterogeneous, the points made by Dennin (2016) represent a clear, if not exaggerated example, of the implicit bias that will reduce access to PrEP.

These fears about PrEP users as either irresponsible or vulnerable are evidently not exclusive to health practitioners; users noted mixed reactions to PrEP and a subsequent change in 
their perceived treatment by peers. Users seemed to indicate some very mixed reactions to PrEP. Though users report that they have routinely convinced their friends to become users, they also note a great deal of resistance from their peers. It is not clear if this theme constitutes a barrier to use for those enough to stop using the drug simply to avoid stigma.

Eric notes that he is constantly suspicious of being "silently judged" by his peers. He writes that despite the likelihood that his fear is "all in his head" he senses judgment as an "irresponsible or dirty or promiscuous" person, especially among his friends with long-term partners. He notes that he has had relatively positive experiences with sexual partners, having told them that he is on PrEP. Contrary to his description of his friends as "uninformed" he frames one positive sexual experience as a man who is "intelligent, informed, open, and mature." Though he notes that this is a rarity in what he calls "Canada's MSM communities."

Similarly, "misinformation" is a concern brought up by non-user 004 in our discussions. They are more concerned about negative perceptions by persons outside the medical community than in the medical community. 002 notes that misinformation is a problem for him as well. He says that when it comes to stigmatized topics he feels more comfortable when the information presented on the topic is accurate and truthful, because when it is not accurate it begins to "hurt people." 004 echoes this issue. They note that when you google PrEP you get lots of misinformation. Though in our discussions, I pointed out him to that there is accurate information online as well. Regardless, 004 points out, "when someone is going to think negatively about me taking PrEP they are going to look for...”as 004 pauses 001 adds, "something to reinforce those beliefs." 003 notes that perhaps this might be due to sex-negativity in the gay community. 003 describes himself as a sex-positive person. He says that these 
negative people would be the opposite, they are against sex and would judge people who are taking PrEP because they are against sex.

This experience is echoed by user James who notes that his experience of discussing PrEP with his peers varies from positive to negative, with not much in between. He notes that he is "constantly" discussing his PrEP use with his friends. His "best friend" is incredibly supportive and is now in the process of becoming a user himself. On the other hand, he has a good friend who is not as supportive. Whenever James discussed PrEP with this "good friend" James feels like he is being "slut shamed." This bothers James because his friend is also in an open relationship and has far more casual "hookups" than James and his husband. James sees himself more-so into using sex as a way of building solidarity between members of his community of "other like-minded cubs and bears." James is irritated by this friend because when they discuss PrEP his friend is quick to question how effective PrEP is, despite James' perception that his friend does not get tested enough to hold the moral high ground in such a discussion. He finds it interesting that the people he talks to who are most interested in shaming him are his friends who often do not know their status or get tested as often. James feels there is a lot of slut shaming in the gay community, likely stemming from "self-hatred." People, he argues, do not want to know their HIV status for fear of being labelled sexually active or a "slut".

It is important to note that users could all identify at least one instance where a peer reacted negatively to their use of PrEP. This is unsurprising as one recent study found as much as $80 \%$ of their participants held negative views of PrEP users in some way (Golub, Gamarel \& Surace, 2017). If this statistic is at all accurate, this $80 \%$ of persons holding a negative view of PrEP and PrEP users is a substantive number of people 'resisting' PrEP (Wyatt, 2003). This is particularly alarming if James' narrative is to be taken seriously. Speildenner (2016) argues that 
the emergence of epithets for PrEP users such as the "Truvada whore" among general slut shaming are part of a framework that encourages shunning both HIV-positive persons and PrEP users for being "sluts." This framework is problematic Speildenner (2016) argues, as it shuts down discussions about safe sex between gay men. This is key, as it may affect the spread of HIV by shutting down discussions of safer sex between users and non-users.

Characterizing PrEP users as at risk, therefore, seems to pose a problem for users and non-users alike in the foreseeable future. In particular the fact that this characterization of replacing condoms with PrEP as irresponsible translates into guilt around their sexual preferences. It is evident that PrEP users are required to carefully manage their identity as condom non-users with the stigma associated with eschewing that established method of HIV prophylaxis. For example, Eric is careful to point out that though he has used PrEP to replace condoms he is not anti-condom. He knows we all "should" wear them, and he still does when he is "being good." He does not even mind wearing them all that much. Nonetheless, he felt an aversion to them, and given the statistics, he states, he is not alone in his aversion. Eric sees PrEP as a way to reconcile these two forces within him. It allows him to indulge his "primitive," natural side with his logical, medically trained side.

Eric's analogy perhaps fits more closely with the fears of those who are convinced by the disinhibition hypothesis. However, the motivations to use PrEP are varied. Vince and Mark on the other hand use PrEP to assuage their anxieties about HIV. Vince, for example, writes that he has always been concerned about HIV infection. He grew up in the 1990s and was well aware of the danger of HIV. Ever since he has become sexually active with men, just over a decade ago, he has been very selective about partners. He thinks he has probably had about 20 partners in total over those 11 years and only five of which with whom he has engaged in penetrative sex. 
This was not in line with his attitude toward sex, which was generally "liberal" and "explorative" towards sex. Because condoms did not work for him, he only engaged in anal sex with a fraction of his partners (maybe as few as five). Despite these precautions, he was constantly afraid of contracting HIV. This fear contributed to his anxiety disorder.

Similarly, Mark notes that his choice to take PrEP was related to an "unfortunate" sexual encounter. During a threesome, he had neglected to wear a condom, which went against the rules his partner and he had set out. His partner was upset with him, and Mark felt awful for not wearing a condom. This was not the first time he had not worn condoms. While his adherence had been fairly consistent as a young man, Mark noticed his condom usage had "started to slip" and he noted that he increasingly disliked using them. After this event with his most recent partner, he sought post-exposure prophylactic treatment and "vowed to acquire PrEP from that day on." By 2014, Mark learned it was possible for Canadians to get a PrEP, which he strongly considered. He knew he was engaging in high risk sexual activity and was vaguely aware that he would eventually contract HIV someday.

As previously stated, key to the disinhibition thesis is the sense that the chance to forgo wearing condoms would correspond to an increase in high risk practices, raising the odds of contracting HIV. While we can see how this arises in Eric's narrative, it is clear that the decision to take PrEP is made once a user has come to terms with their risk profile. For example, PrEP user James' marriage has become increasingly open over time. For the first four years of their marriage they were monogamous. However, once they started making more friends in the "Toronto Club" scene, they found themselves becoming friendly with other "cubs" and "bears." What started out as fairly innocent kisses on the dance floor quickly escalated to "hookups" and to "hosting" group sexual encounters. These parties, he explains, are usually sites where 
recreational drugs are being used. As a result, condoms are sometimes not on everyone's mind. Realising that their risk level had changed, one day James and his partner sat down and decided that they either needed to start using condoms more frequently or maybe think about going on PrEP. While this may seem akin to the alarmist accounts made by persons like Dennin (2016), it is important to note that, users are evaluating their own risk of HIV and compensating, as opposed to using PrEP to engage in risky behaviours.

Indeed, it seems from the accounts of users, they agree with Young and McDaid's (2014) assertion that PrEP use would represent a positive step "in response to risk of HIV because condoms were never or rarely used" (211) by PrEP users anyway. As Eric explains, PrEP is like the safety net under a tightrope walker:

The acrobat isn't supposed to fall when doing tricks in the air, but sometimes they do, and the net is there to make sure that in the off-chance this happens, they don't fall to their death and go splat in front of a bunch of eager (and now traumatized) children.

The "safety net" seems to be an apt metaphor for PrEP. Rather than thinking of it as a "free pass," to engage in wanton promiscuity, users discussed using PrEP proactively, knowing that their sexual practices put them at risk for HIV. Perhaps most importantly, the desire to use PrEP extended beyond persons interested in high risk sexual practices. Users and non-users alike, all with varying risk profiles indicated they were interested in taking PrEP; however, not with the intention of engaging in any new sexual practices. Rather, persons using PrEP discuss how the sense of safety allowed them to feel less anxious during sex, knowing that there was a "net" on which to fall.

Thus, the characterization of PrEP users as at substantial risk due to their condom nonuse not only seems to mischaracterize how PrEP users understand their sexuality and HIV risk, but serves to alienate them from their community and makes accessing sympathetic healthcare 
difficult. Though disinhibition and concerns with respect to increased possibility for HIV transmission are certainly issues worth considering, it is important to note that these discussions can be informed by prejudice about MSMs that mischaracterize PrEP users as deviants or helpless, as opposed to the highly aware sexual actors that they are. At the same time, characterizing PrEP users as necessarily high risk diverts attention from the reality that, as James points out, many persons who would benefit from using PrEP do not do so because they either do not see themselves as at risk or are reluctant to associate themselves with the stigma of being at risk. The unfortunate side effect of this characterization then, is that those who are in fact now at the highest risk are those who continue to engage in "high risk networks" with only sporadic condom use to ward off infection.

\section{Barrier: Side Effects and Weight Gain}

Finally, side effects were a barrier perceived by some non-users, that might keep them from taking PrEP. One side effect indicated in the Truvada product monograph is the potential for "fat redistribution." Though it is not clear if Truvada causes fat redistribution, persons taking anti-retroviral medications, including Truvada, have experienced "increased amounts of fat in the upper back and neck ("buffalo hump"), breast, and around the trunk." (Gilead, 2016 p.8).

Though the incidence of this side effect is rare and it is not certain if it is in fact a side effect of Truvada, the potential for "weight gain" was the most significant side effect noted by participants. Weight gain may itself constitute a reason for rejection or resistance to PrEP as a technology. Studies of contraception, for example, have indicated that a large number of respondents noted that weight gain was among their key concerns with taking oral contraceptives (Emans et al., 1987; Gupta, 2000) that affected contraception use (Himani et al., 2007). Similarly, PrEP may be rejected by persons who need to maintain a particular body type. Though 
this side effect may not be pervasive, for some it may be enough to end their regimen. We can understand this effect as one of many new and unexpected interactions between PrEP and relations not necessarily inside the constellation of HIV prevention discourse.

Non-users 001, 002 and 006 note they are concerned about 'medical side effects,' in particular weight gain. 002 supposes, if he was having more casual sex than he is currently he would consider taking PrEP. But as he is not, he believes that PrEP might do harm to his liver. 001 continues and says he is concerned about the chance of weight gain from the pill. He has a history of bulimia and has struggled with his weight due to medical conditions. He sees the chance that PrEP might result in weight gain as a serious barrier to use. This is echoed by 002 who is also recovering from an eating disorder and sees the potential weight gain from PrEP as a potential trigger. Though this was not as lengthy a discussion in focus group two, 008 notes that he is not interested in a drug that has the potential for weight gain.

Our relationship with therapeutic drugs are largely framed through its side effects. Usually framed as negative or unwanted effects of a drug, side effects are the concomitant effects of a drug that occur alongside the therapeutic effects. The side effect is perhaps the most salient way drugs communicate in their relationship with the user, reminding us that the drug is not "a magic bullet" but a lively chemical interacting with our bodies on its own terms (Gangon \& Holms, 2016). The drugs used in PrEP are lauded as largely side effect free, for most people, and many of the side effects that do occur usually dissipate after the first few weeks. Side effects are experienced very differently from person to person, which in turn impacts their subjectivity (Collins, 2016; Gangon \& Holms, 2016; Fraser, Valentine, \& Roberts, 2009). Though Frankis et al. (2016) note that side effects were a significant concern for their group, side effects were not 
the most salient topic brought up by my research participants ${ }^{9}$. No user reported noticing any negative side effects, a few were even surprised at how well they tolerated the drug.

\section{Is PrEP Configured Correctly?}

Indeed, it appears that PrEP may not be specified for use in the most efficient way. Persons who might benefit from PrEP note a number of reasons why they cannot or otherwise might not have been able to access PrEP. Echoing Holt's (2015) findings, designing PrEP as a drug for people at risk paints the ideal PrEP user as either vulnerable or reckless, a designation that limits access and ultimately the effectiveness of the drug as an HIV prevention technology. Rather than conform to either of these categories, it seems that the successful PrEP user is not necessarily vulnerable or reckless but conscious of their risk profile and taking active steps to remedy that situation. These findings are consistent with Holt's (2015) suggestion that PrEP users continue to resist the association of being 'at risk' with being irresponsible.

Therefore, while the points made by those who forward the disinhibition hypothesis should be taken seriously, as PrEP will no doubt continue to change the dynamics of HIV transmission, it may do so in ways that are perhaps not well described by "disinhibition." In fact, continuing to forward PrEP as a drug for irresponsible persons might be harmful. As PrEP is part of a larger treatment regime, requiring regular contact and supervision with medical practitioners, the theme of resistant medical practitioners poses a significant barrier to PrEP as a successful preventative technology. Inconsistencies in access and willingness to cooperate with aspiring PrEP users means that a number of users will be written out of the process, despite the

\footnotetext{
${ }^{9}$ This may be because my sample size ( $\mathrm{n}=6$ users and 9 non-users) is significantly smaller than those of Frankis et al. (2016) who surveyed 690. Though they did not survey any users.
} 
patient's sense that they might benefit from the treatment. This concern is shared by both users and non-users who note that they have/had little control over the treatment.

Another aspect of the medical system that is of significant concern comes in part from the cost of treatment and the reliance of younger users on their parent's insurance. Users overall are less concerned with cost, presumably because they are gainfully employed and/or insured, as was the case with Eric. Non-users are far more preoccupied with the cost of treatment, noting that they may be able to start treatment with the support of their parents. However, some non-users note that disclosing their desire to become a PrEP user would cause undue strain on their relationships or risk changing their relationship entirely. Non-users are not interested in being associated with the "at risk" category required to become a PrEP user, let al.one the insinuation that a person who is at risk is also necessarily inattentive to that risk. As Holt (2015) argues, the ability to manage disclosure is one of the key characteristics of an effective PrEP user. Though, contrary to Holt (2015), the concern with disclosing PrEP use is not that they are afraid of being mistaken for having HIV or disclosing their same-sex sexual proclivity, but the association with HIV such that they are seen as high risk and/or being reckless, possibly resulting in the loss of resources from parents, as evidenced by 001 's discussion that his parent's discovery of his PrEP use would signal a social "death".

Finally, it is worth noting that weight gain is a significant concern for a number of nonuser respondents, many of whom noted that they suffer from eating disorders and fear any weight gain from PrEP would likely "trigger" their disorder. This side effect is particularly interesting as it has especially clear social consequences. Similarly, in studies of contraception use respondents noted that weight gain was among the key concerns with taking oral contraceptives (Emans et al. 1987; Gupta, 2000) that affected contraception use (Himani et al., 2007). Whether or not the 
potential of weight gain might affect uptake is unclear, however, if these concerns are any indication, then PrEP may exclude users who need to maintain their preferred body aesthetic.

Before moving on, reconsider the parameters I have drawn in order to frame this discussion. Assemblages are made up of a complex interplay of matter and meaning. In order to understand how inscription corresponds to user groups, we assumed that all MSM are the same and are disassociated by this inscription. I noted at the beginning of the chapter that in order to understand whether or not PrEP will become a significant intervention, we should assume that all persons identified as a target user by Gilead is an intended user. Moving forward on the assumption that all MSM might experience PrEP equally, we determined a number of barriers that might make PrEP an ineffective technology, by dissociating a number of potential users. Of course, this is not the only way that PrEP interacts with the MSM communities; PrEP is almost certainly going to redefine how persons engage in a number of relationships informed by other established systems of sexual hygiene. 


\section{Chapter 5 \\ Prophylaxis and the Mutability of HIV Risk}

Though barriers to access and use are a significant threat to PrEP's success, these are not the only problems brought up by my participants. This chapter addresses the relationships outlined by my participants between PrEP and each-other. In this chapter, I compare the motivations for use and non-use to determine how PrEP is interpreted as part of relationships between queer men. As already introduced in the previous chapter, there is a strong possibility that non-user and user groups are made up of several heterogeneous groups, whose interpretation of PrEP differs. In addition to differing social location and levels of access to PrEP, my participants also envisioned PrEP differently. My participants outlined several scenarios where PrEP could be used differently. The stability of these communities and their interpretations of PrEP have implications for its future as an HIV prevention technology. Wyatt (2003) notes that understanding the history of a technology exclusively through the lens of users misunderstands the role of non-users as a significant shaping force on a technology.

Wyatt notes that understanding a technology should not just include persons who interpret a technology as useful but those who actively resist it as well. In addition to those who, for one reason or another, cannot access the drug or those who have involuntarily been ousted from their user-status, Wyatt (2003) argues that it is important understand why non-users resist or reject using a technology (76). "Resisters" and "Rejecters" are non-users who either stopped using a technology of their own accord or have never used the technology because they choose not to (Watkins, 2011). As PrEP remains a controversial drug, understanding how and for what reasons PrEP is resisted by otherwise ideal users is key to understanding PrEP's role in HIV prevention. These reasons for use and non-use, the tensions around conformity and resistance point to emerging forms of sociality and novel collectivities around use and non-use (Collins, 
2016). As we have already outlined in the chapter above, the construction of PrEP as a technology for "whores" serves to dissuade several would be users from pursuing treatment. The way respondents interpret each other and other social groups they interact with will also bear on the success of PrEP in its current form.

Rosengarten and Michael (2010) write that PrEP is an emerging biomedical technology that should be understood as part of an ontologically open assemblage, made up of interwoven and dynamic heterogeneous actors, as opposed to stable and distinct objects. These communities overlap and diverge as their needs and interpretations of a technology change and diverge. According to Flowers (2001) HIV has experienced at least three ontological shifts since the outbreak, as technologies emerged to detect and manage viral load. Each socio-technological shift has corresponded to novel emergent social groups, with norms, signs and symbols associated with their particular community. Communities like HIV-positive and negative, Flowers (2001) notes, emerge as a direct result of the HIV antibody test. These categories have staying power and, once established serve to help form social groups that may last longer than the referent artefact itself. Therefore, though I shall approach the HIV assemblage as open and dynamic, I do so understanding that this dynamism is not uniform.

The most salient reformulation of queer sexuality enabled by PrEP revolves around which prophylactic technique my participants prefer. PrEP is entering a system of HIV prevention that currently consists of two methods of HIV prophylaxis: serosorting and condom use. I will frame how PrEP is apt to change relations between queer men by looking at how PrEP is related to each of those prophylactic methods. Dividing users and non-users in this way gives us three groups, two groups with discernable interpretations of PrEP and a third group which is far more amorphous and points to the variable interpretations and open ontological status of 
PrEP as a technology. Non-users and users alike have clearly delineated interpretations of appropriate PrEP use. While users are interested in using PrEP to fulfil fantasies of condomless sex, non-users were far less interested in going without condoms. Only one non-user came forward that they wanted to maybe use PrEP to replace condom use. The rest noted that though they have engaged in condomless sex, it is not something that is necessarily a source of pride or something that they plan on repeating again. Responses in this section vary as non-users reflect on past experiences and imagine themselves in new ones as they measure themselves up against each other and struggle with notions of stigma and responsible sexuality. How their understanding of self interacts with their sexual practices sheds light on the complexity of the relations on which PrEP stands to bear upon.

\section{PrEP Use: As a Complement to Condoms}

Non-users in this sample were all interested, if not enthusiastic at the prospect of using PrEP. Only 005 and 006 said that they did not want to start taking PrEP. 005 and 006 both note that they would simply prefer condoms over taking a pill — though upon further consideration, 005 noted that she was interested in mixing the two. Mixing PrEP with condoms seemed to be the prophylactic method preferred by non-users. The main interest in PrEP for my sample of interested non-users is the added protection afforded by PrEP when used with condoms. 001 was especially excited at the prospect of a preventative measure that allows him "to do something" to prevent contracting HIV. Similarly, 001 explains though he is aware that he should be using condoms and in a "perfect world" he would; however, "that's just not the reality of the world." Echoing 001's sentiments that PrEP would be useful when things do not go to plan, 009 notes that he would prefer both condoms and, in the "event of a condom failure there is at least one 
other backup failsafe to ensure that nothing will go wrong." As part of another response he echo's this sentiment adding, that in that event he knows he would not have to panic as much.

In focus group one, non-users noted that despite always intending to use condoms they were inconsistent, in their condom use, remarking that several contextual factors bear on their decision to use condoms. Both 002 and 003 note that they have had encounters in the past where a condom was not negotiated, leading to some anxiety about their judgement. 002 says that he is a "really big fan" of condoms, he has a lot of them, "a stockpile, basically" that he keeps in "weird places" because, he explains, he never knows when he is going to need a condom. He does this because he feels safe sex is important to him right now. While he could see himself having condomless sex, this is highly unlikely and he would only do so in a committed relationship with a person who is STI negative. He realizes that this answer could change if he was on PrEP, but he is not sure. 002 notes that he has had condomless sex in the past but he is a lot more cautious in his future sexual encounters because he does not want a serious relationship but wants to "still get laid."

In the second focus group condom use and responsibility arose again as we discussed several hypothetical interactions they may have with a PrEP user. Prior to this scenario, 008 noted that he might use PrEP to replace condoms entirely. Though it is worth noting that he does so with a reluctant tone, as if he was testing the tone of the room. I ask if 008 's point resonates with the group. Though not articulating his disapproval in so many words, 007 groaned as if in condemnation. This groan was not lost on 008 . Later, I ask 007 how he would respond to a person on PrEP asking him to forego a condom, he groans again and 008 laughs, remarking that it seems 007 is changing his mind. 007 notes that he has never had sex without a condom so he does not know if it something he enjoys, but he would probably need to know the person for a 
while for them to engage in condomless sex. He says he is open to the idea, but in this situation, 007 is not interested in foregoing the condom. He likes to think he would be able to say 'no' even if things got "hot and heavy."

This interaction points to an interesting disagreement between the desire to use condoms and the actual practices of condom use. Though 007 notes that he prefers using condoms, he is aware of the potential for misuse. 008 seems to imply that 007 is a bit idealistic in his sense that he will always be willing or able to negotiate condom use. 007 even appears to doubt this as he notes that he hopes he will be able to continue using condoms despite things getting "hot and heavy." A problem paralleled in Flood's (2003) discussion of condom use by heterosexual men and further discussed by the PrEP user, Eric.

In a study of rationales for condom non-use, Flood (2003) notes that one narrative related to condom non-use is that a sexual encounter might get too "hot" for their participants to effectively negotiate a condom. He explains that in Western conceptions of sexuality "sex is figured as a fundamentally irrational and ecstatic domain" in which condoms represent "the intrusion of the practical, responsible and mundane into a space that is impractical, irresponsible and ethereal." (360) These ideas are also more likely to come up, Flood argues, in "one-off sexual episodes," “one-night stands" or when intoxicated. This is reflected in 007's later comments that he would "feel more secure sexually... in a sexual encounter with someone at a club or something, that like, I'd know I had that barrier a bit, I'd still get tested, but, like, I'd be not... like... as scared." This is echoed by Eric who explains that, though he does not see himself as necessarily at risk, he enjoys the added protection from his more "animalistic" tendencies. Though Eric articulates this the most clearly, this issue is brought up by Jason and Marc also as one of the key reasons they became PrEP users. 
We can discern from these discussions our first relevant subdivision of our user and nonuser groups: Persons who use PrEP as a safeguard against contracting HIV, to be used in tandem with condoms. The problem for which PrEP is seen as a solution in this case is that condoms are not always available or used correctly. Potential PrEP users see PrEP as a solution to the problems posed by inadvertently failing to use a condom, or "slipping up." These "slip ups," are a source of anxiety for my participants, both for non-users and for users prior starting PrEP. Thus, offering PrEP to persons to use in addition to condoms, may not just reduce their HIV risk, but help relieve some of the anxiety that a condom was misused or not negotiated in time.

\section{PrEP Use: As a Replacement for Condoms}

Though non-users were more interested in the added protection afforded by PrEP when used alongside condoms, users noted that they used PrEP because they were either highly inconsistent condom users or preferred not to use condoms at all. The latter group of condom non-users, referred to as "barebackers" refers to a group of potential users who wanted to engage in condomless sex or have been engaging in condomless sex because it is their preferred type of sex. Though non-users noted that they engaged in condomless sex when in a monogamous relationship with their partner, this group engages in condomless sex, regardless of the type of relationship. Though PrEP is not currently indicated for use for this type of sex, this group likely stands to gain the most from PrEP as an intervention. Not simply because they constitute one of the most substantial risk profiles of any MSM subculture, but as we will see, because this subculture with PrEP in hand may redefine the political landscape of the gay community.

Though non-users are certainly enticed by the added protection afforded by PrEP, they were not interested in using PrEP to replace condoms. This is certainly not necessarily the case for a number of current PrEP users who offered graphic fantasies of "raw" sex and a sense of 
release associated with taking PrEP. Consider the narratives of Christian, Vince and Eric:

Christian, for example, notes that he became aware of PrEP while pursuing his personal fantasy for condomless sex. Barebacking was a fantasy of Christian's for some time prior to becoming a PrEP user. He calls condomless sex his "ultimate taboo," a "hot fantasy" and "pure escapism." Before starting PrEP, Christian had been living his sexuality vicariously through a "bareback hookup site." He lamented that to indulge in his fantasy without PrEP would be met with "dire" consequences." It was on this website that he was first acquainted with PrEP, through posts written by a pro-PrEP HIV activist and he began the process of becoming a user.

Though Christian is aware of the "dire" situation he may put himself in, he finds intense pleasure in having sex without a condom. Similarly, Eric writes:

I'm still somewhat self-conscious in admitting and discussing this, as it's still not socially acceptable really and goes against all public health initiatives since forever, but barebacking is fucking hot. I really struggled with this dilemma and my innate attraction to it while considering PrEP, so I can say that I've really challenged myself with questions regarding unprotected sex and forced honest and thoughtful answers and considerations from myself..."

It is clear from Eric's narrative that he sees himself not simply as adapting to the needs of his partner, but as actively interested in not using condoms. Eric's choice to use PrEP is made in relation to his understanding of the acceptability of unprotected sex. He struggled with his identity as a person who likes condomless sex. He saw himself as an "intelligent, responsible, medically educated and trained adult" who should not be attracted to barebacking. Yet, he notes that he "slips up" far too many times for his condom non-use to be truly accidental.

Contrary to the group above, many PrEP users indicated that they were using PrEP to engage in bareback sex. While those who indicated they were interested in using condoms with PrEP in the event of a condom malfunction or miscommunication, this group intends to use PrEP to replace condoms entirely. This use for PrEP has been criticized by scholars like Poicus (2016) 
who argues that using PrEP in this way allows a select few privileged individuals to engage in condomless sex without the political obligations associated with barebacking. By shifting the boundaries of immunity, Poicus (2016) argues, this use for PrEP allows the user to eschew established norms around queer sexual hygiene and inhabit the heteronormative sexual mainstream. While I do not intend to argue against this point, using PrEP to replace condoms certainly evidently part of the motivation to use PrEP. However, as we will see below, PrEP is not simply used passively by users, but is an active agent in shifting relations between multiple communities of sexuality with varying interpretations of contagion and risk.

\section{PrEP Use: Serosorting}

Though some participants mentioned they used condoms exclusively, while some others indicated they never used condoms, most participants realistically fell somewhere between those two poles. Most of my respondents could be called "contextual condom users", or people who use serosorting as a method of HIV prevention. Serosorting emerges as a prophylactic method around the time the HIV antibody test became widely available to the public (Flowers, 2001). Beginning in the early 2000s, a new safer sex technique called "test and tell," or in other circles "serosorting" came to be the preferred safer sex method in some MSM communities (Flowers, 2001; Halperin, 2016). The idea was that, due to the availability of regular testing, "seroconcordant" couples could safely engage in condomless sex, as long as they kept tabs on their own status and the status of their partners (Halperin, 2016; Flowers, 2001). This method of "negotiated safety" or "strategic positioning" in the case of barebackers (Dean, 2009, p.13-14) is useful so long as the partners communicate as it relies on trust between parties who are negotiating a complex semi-verbalized process of obtaining consent. 
Although non-users enjoyed using condoms, they noted that their choice to use a condom was context dependent and therefore they would appreciate the added protection PrEP affords. Building on his prior comments, 002 notes, however, that there are factors that would affect his preference for condoms. While 002 is clear that he does not like condomless sex and would terminate his encounter should that happen, he slowly reconsiders this positon, imagining that, if his partner was attractive enough and did not want to use condoms, he might consider not wearing a condom. The contextually contingent nature of condom use is echoed by 003 who explains he has engaged in several sexual relationships where both partners agreed on whether or not to use condoms. He notes that there are a few occasions where he and his partner have stopped using condoms, due to being in an exclusive relationship, but at the same time he explains he has negotiated relationships that were exclusive relationships in which condom use was always expected, regardless of exclusivity.

Though many of my participants are not averse to using condoms, they are aware of times when condoms may not be necessary or indeed desirable. Understanding how condoms are used contextually, points to exactly why it is important to think about PrEP through the lens of dynamic, interrelated and open systems. The motivations to engage in condomless sex are multiple and hinge on the meaning attributed to that relationship. These meanings are largely dependent on the type of relationship and the connotations PrEP might imply, given the boundaries of that particular relationship.

As I discussed above, PrEP affords uses as a prophylactic that condoms cannot. As such, it serves to reason that it also cannot be expected to work as a condom would. One such affordance is its visibility. Race (2016) argues that condoms provide "mode of ordinariness in a situation of unendurable and ongoing crisis" (23), where one can effectively distance themselves 
from the HIV epidemic. On a practical level, the visibility of condoms allows them to function as a symbol of safety, a signal that the interaction is low risk, even if the participants are unsure of their HIV statuses. Condom use, therefore allows one to forget or ignore HIV so long as the condom is employed. How that symbol is interpreted, however, is perceived differently according to the type of relationship in which the user is involved. Rather than discuss PrEP relative to their HIV risk, my participants discussed how PrEP might be perceived within their relationship. These relationships and the acceptability of each prophylactic method as described by my participants were highly varied, some of which I was able to summarise in the following table:

\begin{tabular}{|c|c|c|c|}
\hline Relationship Type & $\begin{array}{c}\text { Neither condom } \\
\text { nor PrEP. }\end{array}$ & Condom use only & PrEP only \\
\hline $\begin{array}{c}\text { Committed } \\
\text { relationship }\end{array}$ & Appropriate & Appropriate & Dependant \\
\hline Open relationship & Inappropriate & Dependent & Appropriate \\
\hline Casual partner & Inappropriate & $\begin{array}{c}\text { Appropriate (though } \\
\text { slip ups occur) }\end{array}$ & Inappropriate \\
\hline Novel partner & Inappropriate & $\begin{array}{c}\text { Appropriate (though } \\
\text { slip ups occur) }\end{array}$ & Appropriate \\
\hline Exclusive relationship & Appropriate & Appropriate & Inappropriate \\
\hline $\begin{array}{c}\text { Polyamorous } \\
\text { (multiple) relationships }\end{array}$ & Dependent & Appropriate & Appropriate \\
\hline Clients & $\begin{array}{c}\text { Inappropriate } \\
\text { (though assault } \\
\text { may occur) }\end{array}$ & Appropriate & Appropriate \\
\hline Unfaithful partners & $\begin{array}{c}\text { Inappropriate } \\
\text { (n) }\end{array}$ & Dependent & $\begin{array}{c}\text { Dependent (though } \\
\text { likely inappropriate) }\end{array}$ \\
\hline
\end{tabular}

Table 1. A typology of appropriate safer sex techniques according to relationship type

The sheer number of possible relationships and appropriate prophylactic methods points to the complex interplay of factors that impact and are impacted by PrEP beyond HIV risk. It also demonstrates the role of situational knowledge in the emerging attributions made to PrEP across different contexts. This list is of course not definitive, as it only reflects the at times conflicting 
opinions of a small number of respondents and their own opinions and personal experiences.

This chart is useful for the purposes of this thesis in that it helps visualize interesting points of contention and solutions brought to the fore by PrEP. Though these various types of relationships will themselves bear on the success of PrEP as an HIV intervention and therefore warrant further study, for my purposes we shall discuss these relationships fairly broadly as PrEP's influence on systems of serosorting.

Non-users in both focus groups noted that they reserved condomless sex to serious relationships. Similarly, PrEP user Vince writes about his relationship with condoms prior to PrEP:

Even though I loved bareback sex and porn, I was terrified to do it and even resented people who engaged in the bareback lifestyle. I judged them for being irresponsible and spreading HIV, but I was also curious and envious. I only had bareback sex with longterm partners and I avoided anal sex altogether with most casual partners.

Mark also notes that there is a relationship between intimacy and condom use, echoing most other users, who report condoms as a barrier to intimacy. Though it is worth noting that in many cases, a user's definition of intimacy does not necessarily relate to a committed relationship. In fact, my participants listed several different forms of relationships for which PrEP may or may not be appropriate. 003 notes that there is something about condomless sex that puts it on what he calls a "spectrum of intimacy and pure physicality," saying that there are people who just prefer barebacking as their type of good sex (Harvey, 2009). He notes further that there is a connection between intimacy and barebacking that makes it "special," reserved for only a few types of relationships. Specifically, a sexual relationship in which he and his partner have built trust. Similarly, 009 notes that to not wear condoms is the "ultimate form of intimacy" between him and his partner. 009 explains that because it is so intimate, he would never purposefully forego wearing a condom with a casual partner. Therefore, if he were "hooking up" with a 
partner off Grindr, there would have to be condoms. 009 says that he would forego condoms, only if he has known his partner for some time and has built a trusting relationship.

Interestingly enough, there is little difference in these rationales for condom non-use than those reported by heterosexual men in studies of condom use and non-use. Flood (2003) notes that in discussions of condom use, love and trust are themselves understood to be prophylactic. This is consistent with findings by Katz et al. (2000) and Critelli \& Suire (1998) who note that condom use is negatively correlated with more serious monogamous "positive" relationships, as well as with age ${ }^{10}$. Further, Corbett et al. (2009) note that condom non-use is itself a strategy used to help establish a serious relationship. They note that the symbolic value of condom nonuse helps establishes trust and can be used to convey monogamy, a need that often-outweighed health concerns. Similarly, Mustanski and colleagues (2014) found that trust was central to some young gay and bisexual men's decision to use condoms. Though similar behaviorally, it is important to point out a distinction in the affective experience of condomless sex between heterosexual and queer persons. Condom use has been a "celebrated" and "integral" tool for gay sex since the 1980 s and the only socially acceptable HIV prophylactic method for the last 40 years (Spieldenner, 2016). However, that method of prophylaxis rubs up against monogamous and abstinence prophylactic methods that predate condoms as an AIDS prophylactic (Epstein 1996; Flowers, 2001).

These notions of trust are troubled by PrEP for a few reasons. Firstly, it divorces persons and practices otherwise at a higher risk of contracting HIV from being "unsafe." Secondly, PrEP use can be interpreted as a sign of infidelity or, conversely as a signal to a partner that they are untrustworthy. Trust was a significant discussion point for both focus groups and a theme in

\footnotetext{
${ }^{10}$ I only include this as my PrEP user sample was for the most part older than my non-user sample.
} 
number of user's accounts. For example, 006 envisions that he would start taking PrEP if he was in a relationship with a person he did not trust. This interpretation of PrEP, as a defense against a partner they do not trust is echoed by 003 , who had just such an encounter with a previous partner. 003 tells us that his boyfriend started taking PrEP because he felt that 003 has behaved in such an unsavoury way as to make him consider taking PrEP. 003's boyfriend at the time thought that 003 was going to get HIV and give it to him. Though they had agreed on seeing other people, in addition to each-other, his boyfriend was so sure that 003 's judgement was poor, that he decided he was going to start taking PrEP. This had an intensely negative effect on their relationship and ultimately contributed to their break up. He notes that while he wants to take PrEP to help reduce the stigma associated with HIV, his partner did the opposite. His partner was so afraid of contracting HIV that simply the thought that 003 could be positive was enough for him to take on the financial burden of PrEP.

PrEP was, indeed seen by some as an unwelcome intervention in their relationship. For example, 008 notes that PrEP would most certainly put a strain on his romantic relationships. He feels like his partner would be wary of him taking PrEP, would question his motives and notes that PrEP would out you in a "certain category" with "particular connotations." This narrative runs along the lines of Eric's narratives that he "looked" like a person who was at risk of contracting HIV. When I ask what he means, 008 responds that "if someone is cheating or you know sleeping around and taking this pill for precautionary measure and they are in a relationship with somebody...and then the person sees these pills" there is a chance for “situations to pop up." In essence, PrEP could be taken as a sign of infidelity regardless of if one's partner was truly unfaithful. 
Thus, when it comes to negotiated condom use, PrEP interferes with established rituals made both along the lines of HIV risk but also along the around ensuring intimacy and trust. In high risk communities, the risk of contracting HIV must be considered alongside risks of seeming distrustful or unfaithful partners (Corbett et al., 2009). These risk evaluations and notions of trust are complicated by PrEP's introduction as it troubles both established signs of safer sex and signs of intimacy. This is because PrEP challenges the symbolic power of using or not using a condom. That is to say, using a condom "out of the blue" in a monogamous, exclusive relationship could be interpreted as a sign that your significant other is being unfaithful by trying to minimize their chance of exposing you to disease. Likewise, seeing a bottle of PrEP can be interpreted as unfaithful or distrustful for trying to manage their HIV risk. For contextual condom users, these two risks need to be delicately balanced.

Evidently, PrEP engages unevenly in relationships where HIV risk, intimacy and trust are negotiated between sexual partners. While it may seem that PrEP use in these cases seem highly contextual, there are unifying themes between these accounts that point to how PrEP may change how sexuality is negotiated. How PrEP interacts with each these negotiations elucidates the role of contagion in how people interpret intimate relationships and evaluate condom use. While my participants provided a wide array of context in which they thought PrEP or condoms might be more appropriate, what is particularly interesting about these negotiations is how similar their accounts of condom use are to those of heterosexuals in the literature, with one key exception. Whether or not they identified their condom use as a risk for either pregnancy or HIV. While my participants universally understood their condom use in light of HIV, their heterosexual peers were far more concerned with pregnancy than HIV. Underscoring this point my participants 
routinely explained that their group identity, rather than their behaviour informed whether or not they were at risk of HIV.

\section{The Multiple Constructions of HIV Risk}

Understanding how PrEP forms and reforms communities as "at risk" requires us to attend to the politically charged agendas that are often manifested in technical interventions. Bowker and Starr (1999) note that classificatory schemes become "enfolded into a working infrastructure," entrenching and further naturalizing those relations until they become taken for granted (196). PrEP emerges as an intervention in an HIV prevention apparatus with a unique history and established social and technological norms. HIV is informed by a biomedical approach that conceived of AIDS as the result of the HIV virus, and (if one were being generous) an "epidemiologically" oriented hypothesis that argued AIDS was multifactorial and varied depending on the risk group demonstrating AIDS-like symptoms. The fact that the first HIV outbreaks, and the vast majority of HIV knowledge generation, occurred in the United States is not inconsequential to the emergence of these two ontological frames. The United States, as opposed to their European counterparts have developed over time a valuation of medical knowledge that is "functionally understandable," open to common sense and conducive to individual management of disease (Patton, 1986). The commodification or, as some might say, the democratization of medical knowledge is perhaps necessary to keep in mind when remembering the controversy that formed and continues to define the HIV assemblage.

Epstein (1996) details the outright denial of AIDS as a heterosexual condition in the early days of the epidemic right up into the mid-1990s. These HIV as AIDS dissenter camps forwarded an explanation that AIDS was not a virus but the result of the deviant practices of homosexual men, such as drug use or "hyper-sexuality." Treichler (1987) notes that the science 
of HIV and its "non-scientific misconceptions" become intertwined in a discourse that continues to linger in both the somatic understanding of HIV risk and in its prevention paradigms. Treichler (1987) notes a number of conceptions of HIV/AIDS that correspond to our current epidemiological categories: In particular, that HIV is largely a gay disease acquired through the depraved sexual acts in which queer men engage (36). The response to HIV/AIDS in Canada closely parallels that in the United States, albeit in a slower and less coordinated fashion (Krever, 1997). Some health care responses to HIV/AIDS in the 1980s (during what Flowers, 2001 calls the "confused period" of HIV risk), reflected these non-scientific misconceptions about the transmission of HIV. These misconceptions made their way into official understandings of HIV risk, and then into screening technologies (Krever, 1997) which once entrenched have proven to be unyielding as technologies of HIV surveillance, despite advances in HIV screening technologies.

As I discussed in the previous chapter, the effective PrEP user successfully positions themselves within the user category by being conscious of their risk profile and by taking active steps to remedy that situation (Holt, 2015). While this is the case, both Holt and my own participants make clear that their individual definitions of "high risk" are highly variable. The comparison of users and non-users in the sections above gleans multiple contrasting lenses through which they define risk and PrEP as a risk mitigation strategy. This risk profile is further troubled by the interaction of PrEP with established symbols of trust and intimacy, such that it adds a dimension of risk that, complicates how risk is distributed across relationships.

Though my respondent's risk profiles varied significantly, their actual rationales for not using condoms continued to parallel those of heterosexual men closely. Like their heterosexual peers, my respondents noted that they were dissatisfied with condoms, interested in maintaining 
intimacy with their partners and had difficulty managing to negotiate safer sex in the heat of the moment. This is no different from the heterosexual male participants in Flood's (2003) study who also engage in a complex system of semi-verbalized messages with their partners to determine safer sex practices. Further, they use similar notions of trust and relationship type to determine the need for condoms. How should we understand the relationship between safer sex practices and HIV risk, in light of these parallels?

One way we can understand how HIV risk is redrawn is along the lines of immunity. Poicus (2016), for example, concludes his recent chapter on the geocorporalities of PrEP arguing that the drug creates a "zone of viral exclusion in at risk bodies" in the global north. Through the consumption of PrEP, relatively privileged queer men are able to forgo the "sexual hygiene practices" that emerged during the AIDS crisis (37). Through immunity, the borders of HIV risk are redrawn. His final line was particularly amusing to me: "It is immunity that separates bare life from barebackers" (ibid, p.37). This is certainly true; the logic of immunity does distinguish those at risk for HIV from those who are deemed not at risk. It seems to me, though a poetic and highly memorable concluding point, "bare life" does not necessarily encompass the richness of PrEP's intervention; though it does call attention to the new and emerging forms of bios that PrEP allows.

Poicus (2016) notes that PrEP allows condomless sex to be tolerated, so long as one is responsible for it. This conclusion does not attend to the connection between identity and these sexual hygiene practices. What is certainly evident from my participants is that PrEP plays a role in defining distinctions between those who are infected, those who are not and those who are at risk. "HIV-positive" as a social category demands of us a particular set of relations. Though these relations are connected to the management of viral load and transmission, they are indeed 
connected to sexual hygienic norms and extend beyond the management of the virus. It extends into the practical logic of HIV transmission and thus into the ontological status of HIV itself.

The role of HIV as a structuring structure is best highlighted in the difficulties

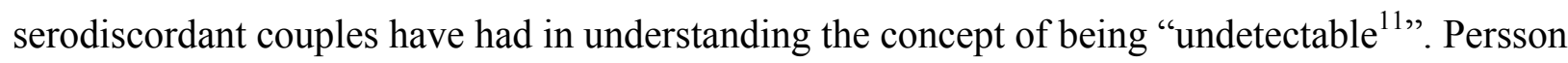
(2012) found that despite understanding the link between undetectable viral load and reduced infectivity, her respondents had difficulties reconciling their own knowledge about HIV with the possibility that a person with HIV could become sexually non-infectious (1072). It was very difficult for participants to divorce the social category of HIV-infected from the possibility that HIV load could be suppressed through HAART to the point they were no longer infectious. For Persson's (2012) participants, HIV was not so much a medical problem as it was an ontological problem. This logic is not lost on my participants, who routinely take into account the social affordances of PrEP before considering the dynamics of the pill in regard to the virus itself.

An unfortunate development of the controversy around AIDS is a conflation of HIV transmission with risk group. Epstein (1996) notes how the forwarding of the Risk-AIDS hypothesis by high profile HIV dissenters like Peter Duesberg, Bryan Ellison and Michael Fomento (the author of The Myth of Heterosexual AIDS) helped cement the notion that AIDS was a risk group disease, not a result of HIV infection. This is important because this notion has had staying power, in part because HIV-risk practices mirrored the more favorable routes of HIV transmission thereby allowing for a conflation of risk behaviours with group membership. Rather than understand HIV as a virus that anyone could potentially contract, these ghosts of

\footnotetext{
11 "Undetectable," refers to an HIV-positive person who due to HAART treatment has suppressed their viral load to less than $40 \mathrm{copies} / \mathrm{ml}$ for at least six months. These people are regarded as no longer sexually infectious. (Vernazza et al., 2008)
} 
controversy past live on in the sense that the HIV virus is transmitted only through risky behaviour within an at-risk group.

Apart from this distinction, my participants' sexual practices/needs do not seem to diverge too far from those recounted by heterosexual participants in other studies. Indeed, studies of condom use seem to indicate little difference in the justifications for condom non-use between HIV-negative heterosexuals and queer men (Flood, 2003; Corbett et al., 2009; Adam et al., 2008). Both Flood's participants and my own note that they take into account trust, decreased penile sensation, loss of erection or difficulty of use in 'the heat of the moment' as the primary reasons for not using condoms. These points, again, are echoed in Adam et al.'s (2008) study of Ontarian gay men's condom use practices. The key difference between Flood's heterosexual male participants and my own is the perception of whether or not their condom use was understood in light of a risk of contracting HIV.

A running theme throughout my narratives was the experience of "coming out" and a simultaneous dawning realization that they were at risk of contracting HIV. This theme seems to indicate a synchronized transition from identifying as "straight" and at risk for pregnancy to "gay" and at risk for HIV, regardless of changes in corresponding sexual practices, apart from the gender of the participant's partner(s). This theme points to a sense that HIV risk is exclusively a problem associated with same-gender sexual practices. Indeed, heterosexual condom non-users in Flood's (2003) study indicate that the risk of pregnancy far outweighed the perceived risk of contracting HIV. They justified their position by explaining that heterosexuality itself was enough of a protection from HIV:

It was not merely an imagined 'heterosexual community,' but the very sexual practices which constitute it, which are rendered AIDS-free in this construction. [Flood's participant] Adam's mention of 'clean sex acts' may represent long standing associations of STIs with dirt and poor hygiene and of homosexual sexual practices as 'unclean' and 
dirty and thus dangerous. (Flood, 2003, p. 364)

This conflation of sexual identity with, dirty, "unhygienic" practices and HIV-risk was not lost on my participants who found their same-gender attraction fundamentally linked to HIV. HIV risk was routinely discussed in the same breath as the act of "coming-out." Christian, for example, noted that he has been using condoms "religiously" since he came out as gay in 1990. Vince, too, noted that his relationship to HIV was framed in terms of his sexual identity. He explains that, "prior to coming out as gay, I had tried sex with girls with and without condoms, and preferred without condoms. The worry with them was pregnancy. But once I came out as gay, I only had sex with men, and HIV became the new worry."

In the above cases, participants identified themselves as at risk for HIV as soon as they came out. This association, however, is not necessarily a personal choice. In some instances, participants were cast into this category of "at risk" by family or members of the medical community as a result of coming out. 003 , for example, noted that it was really hard to come out to his father because his father was "very biased" with respect to the role of HIV in gay life. The first few years of being out were difficult for 003 , especially in relationship to his father because his father had a certain idea of the "type of people who get AIDS." He explains that "I've been told ever since I've come out it's just been people constantly telling me I'm at risk." These are not just medical professionals but his friends and people with whom he had been romantically involved. In contrast to 003,002 notes that HIV has not been particularly present in his life and that "more and more" he is considering himself at risk for HIV. But (presumably in contrast to 003) this risk profile was not so much "ingrained" in him so much as a "dawning realization" of the reality of his sexuality. 
Despite the logic that this HIV risk should be related to particular sets of practices, HIV risk seems to be more or less universal in its application to same gender sexuality. Each of the participants above outlined a very different set of sexual practices and safer sex preferences. Vince notes he became increasing "liberal" with age culminating in his PrEP use and Christian professed a secret desire to forgo condoms prior to taking PrEP. By contrast, 002 notes that he only really likes having sex with condoms and 007 notes he never practices condomless sex. 003 notes that he has engaged in contextual condom use, negotiated with his partner at the time. Each set of practices are objectively more or less likely to transmit HIV. The interesting point is that, for my participants, they understand their HIV risk as universal. Perhaps pointing to HIV risk as an identity issue, as opposed to a behavioural issue. Coming out, thus, corresponds to a shift in a conceptual category from not at risk for HIV to, at high risk of HIV transmission.

It is evident that PrEP is engaging with a community that is largely impacted by HIV; both in the sense that HIV is highly present in this community and that a person's self-concept is framed in relation to HIV risk. This is not to say that heterosexual sex is not itself framed in reference to HIV risk, but that PrEP emerges to meet an underlying need in the queer male community. While Holt (2015) has pointed out that the various definitions of high risk subvert PrEP as a technology, my non-user participants point to a completely different interpretation of PrEP. What is interesting here is that the need for PrEP that appears to extend beyond reasonable risk analyses or HIV prevention at all. Recall 009's point that despite desiring PrEP, he does not meet a single risk criterion. If he was not at risk, why does he want to start taking PrEP? Let us turn now to examine further the competing relations of matter and meaning that may explain 009's seemingly irrational desire PrEP. 


\section{The "Latent Need for PrEP"}

From a health care perspective, the argument for PrEP is pretty straight forward. HIV is a contagious, potentially life threatening, chronic condition that ought to be avoided. Anyone at risk of contracting HIV should take it as part of their desire to preserve their own health as well as the health of the community. This assumption is best encapsulated by the guiding principles embraced by the Joint United Nations Programme on HIV/AIDS (UNAIDS). UNAIDS ${ }^{12}$, s (2014) identifies HIV endemic groups as "those left behind." The UNIADS Gap Report, for example, frames HIV prevention uptake as "dependent on the quality of services as well as the levels of stigma and discrimination by service providers." (118). In other words, HIV is dependent on the quality of prevention efforts and thus HIV transmission is a result of failed prevention efforts. A perspective on HIV transmission that focuses exclusively on social exclusion as the cause of HIV transmission, though indeed an important insight, is shortsighted to the relational factors impacting health and insights about technological resistance or reinterpretations (Watkins, 2011; Wyatt, 2003; Pinch \& Bijker, 1984). Framing the desire to avoid HIV and promote community health ignores the fact that people often do not take health precautions simply because it would seem to be in their best interest.

Though PrEP makes sense from the perspective of HIV prevention, it may not suit the needs of those who should be taking it. Objectively, PrEP is not very useful in the grand scheme of health care. For its cost, it is not half as effective as other lifesaving drugs. PrEP is expensive, vaguely poisonous and only provides protection against one STI. Condoms, on the other hand,

\footnotetext{
${ }^{12}$ It is on the basis of this premise that the IPERGAY PrEP study was justified. I also want to point out that by focusing on this frame I oversimplify a 400+ page report that carefully considers the social life of HIV. The point here is that HIV prevention operates from the perspective of social lack is overemphasized in discussions of HIV risk. This overemphasis has an unintended function of distancing and diminishing persons affected by HIV and thus serves to continue marginalizing those at risk (for a discussion of the nature of "liberal othering" see Young, 2007)
} 
are generally not poisonous to most persons and can be accessed fairly easily at no cost. Further, as Holt (2015) and Rosengarten \& Michael (2009) note PrEP suffers from a largely undefined target group: "healthy" persons "at risk" for HIV. The Centers for Disease Control and Prevention estimate that only around a quarter of MSM would benefit from PrEP (Smith et al., 2015), yet $60 \%$ of MSM expressed a desire to take the drug (Hoots et al., 2016). Christian writes about his surprise at the gay community's reaction to PrEP:

What's amazing is that there was a latent need for it! I wasn't the only one feeling liberated by PrEP. In the last three years, PrEP adoption has been pretty spectacular in Toronto. ${ }^{13}$ As if a dam of fear and shame had collapsed over night (sic) and gay guys felt they could reclaim their sexuality.

Those convinced by the disinhibition hypothesis might read this statement and think that there is a latent hedonistic quality to the Toronto gay scene that makes PrEP well-liked. However, if we consider the way PrEP users discuss their sexuality compared to non-users we can glean another meaning. Specifically, that PrEP is useful in undoing the contradicting risk evaluations around trust and HIV risk that have made both HIV prevention and queer relationships difficult to maintain.

Novel uses of PrEP become clear when comparing how HIV risk is constructed along the lines of sexuality. This paradigm constructs all socially acceptable forms of heterosexuality as inherently prophylactic, while same gender sexual practices are understood as "unhygienic" and thus at risk of infection. This paradigm translates into anxiety for persons who practice same

\footnotetext{
${ }^{13}$ As there are currently no statistics on PrEP uptake in Toronto, it is not clear how extensive this "spectacular" uptake is, in fact. Though anecdotally, accounts from community health providers in Toronto seem to confirm this trend. AIDS Council of Toronto (ACT, n.d), for example, has been keeping tabs on their discussions of PrEP with potential users. They note that in the six months leading up to their most recent report to the ministry of health, they have discussed PrEP with at least 223 different persons. The majority of these conversations were around the nature of PrEP or how best to access it. It is also worth noting that in the United States, where PrEP has been available long enough to gather statistics, PrEP prescriptions in retail pharmacies alone have increased by 523\% between 2013 and 2015 . This is about 50,000 persons across the United States (Bush et al., 2016).
} 
gender sexuality, as all sexuality of this sort is painted with the same risk brush. A fairly universal trend across all my participants is a sense that sexuality is inherently risky. As such, my participants routinely express that they are highly anxious before, after and sometimes during sex. This opinion was shared by persons who exclusively used condoms, contextual condom users and by those who preferred going without condoms. These accounts shed an interesting light on the role of PrEP for our condom user group. Though in some ways, PrEP non-users saw value in PrEP's ability to protect themselves from unsavory characters, this is not the only source of anxiety. For many, every sexual encounter is in itself a risk.

In light of the construction of same gender attraction as a universal risk for infection we can understand why even meticulous condom users want to use PrEP. "Slipping up," is necessarily a traumatic experience, even in the most innocent of circumstances. Consider the discussion of PrEP's role in HIV related anxiety: 003 points out that he has done "extremely regrettable things," things he cannot guarantee he would not do again. If he was on PrEP, he notes that the anxiety related to these types of interaction would certainly be lessened. Later in the interview he explains this position further, "every time I like get STI tested I have a mini panic attack, like, I'm convinced I have made an error in my judgment and I'm going to be HIVpositive." Vince too notes that he was always worried about contracting HIV prior to starting PrEP. He writes that, although he was very interested "and had liberal and explorative attitudes towards sex, I was scared most of the time. I already suffered from anxiety disorder, and the worry about HIV was just one of my many sources of anxiety." This anxiety mostly emerged around novel sexual partners.

Similarly, 009 notes that PrEP would ease a lot of anxiety that he experiences around sex. A point that 007 confirms as a problem he also experiences. For 007 it is not necessarily the act 
of intercourse itself that makes him anxious. He notes that though he might not be anxious during sex, he is certainly anxious before and afterward. He points out that he would be more confident having sex with a person who was on PrEP. It would relieve some anxiety about having sex with a new partner. He knows even without the condom, there would be one more barrier to fall back on. He notes that though he would still go and get tested regularly, he would not be as scared going to get his results.

Thus, while PrEP does shift the borders of risk along the lines of immunity, it does so in a far more pervasive manner than outlined by some studies of the social life of PrEP. My participants discuss a hope for PrEP that extends beyond HIV transmission. This "latent need" for PrEP, I argue, is a contradiction between the sense that all queer sex is equally dangerous and all heterosexual sex is, by contrast, inherently prophylactic. Thus, rather than discuss the disinhibition paradox, I would like to posit a more likely paradox stemming from the ever growing disjoint between risk behaviour and risk group.

Knowledge about HIV has always been multiple at times contradictory. The prevailing knowledge paradigms around HIV/AIDS can be largely grouped into two streams: one that conceptualizes AIDS as a virus spread through a particular set of behaviours and another that forwarded an idea that AIDS was the result of practices associated with particular identity groups (Epstein, 1996). Since the early days of the virus, LGBT patient groups have carved out a role in HIV discourse. Through the involvement of activist groups, HIV/AIDS as retained a collective and individual quality as both a disease of gay men, and of particular practices (manifesting in the problematic MSM catch-all term) (Epstein, 1996; Rose, 2007). Though the virus could very well have remained very lethal without the involvement of queer activists, this involvement has help keep alive the legacy of HIV as, at least in part, a "gay disease." These epidemiological 
categories become increasingly difficult to resist. Consider Mol's (2002) argument that populations, once identified as a medical target, serve to generate the epidemiological norms for that community. In the medical sphere, epidemiology and diagnostic practices make a selfreinforcing loop that serves to generate a population the reinforces the existence of the peculiarities of that condition (Mol, 2002, pp. 130-131). What these narratives point to is the tensions between the competing definitions of HIV as they relate to the biomedical profiles of persons as risk of HIV.

This sense that each sexual encounter could be "the one" runs against the emerging epidemiological patterns that inform how the virus is understood by general practitioners. As we learned in the previous chapter, HIV risk also needs to be performed properly in order to be diagnosed at the level of the practitioner. Engaging myself in the focus group discussion, I shared my own experience of entering the medical system after coming out. In response to 003 's experience of being routinely told by medical practitioners that he was as risk, I shared my own experience. I explained shortly after coming out I began getting tested regularly for HIV. I understood being responsible and sexually active as a gay man meant that I needed to get regular HIV tests. However, my doctor was reluctant to test me because in his eyes, I was not at risk for HIV.

We can see these competing frames of reference for HIV risk in my own experience with the HIV prevention assemblage as a young man. My doctor of course was not incorrect in telling me that I was not at risk of contracting HIV. Statistically, my chances of coming into contact with an HIV-positive person was so low that it would be a waste of his time and provincial resources. In many ways my doctor was right, as I was a 15-year-old, white, middle class, suburban teenager with only a few, equally white, middle class, teenage, past sexual partners. 
However, I was adamant that, as a "gay man," it was imperative that I get tested. Of course, if I were to have consulted the relevant epidemiological literature I would have understood why my anxiety was misplaced. In many ways, my doctor was correct in his reluctance to administer HIV tests. In Manitoba, where I am from, HIV diagnoses in heterosexual persons have increased significantly such that heterosexual sex has come to be the primary route of transmission in the region (Public Health Agency of Canada, 2014).

If they have not already, these competing paradigms of HIV risk come to a head, brought into further contradiction by PrEP. As the epidemiology of HIV continues to evolve alongside new preventative technologies, systems of social organization around the disease will need to change. Of course, the connection between queerness and HIV is a long standing and perhaps largely obdurate set of alliances that co-construct HIV and the MSM communities. These identities become a problem for and/or to be solved by the inclusion of PrEP in the HIV prevention paradigm. In order to truly understand the future of HIV prevention in Ontario, we should consider how PrEP and HIV are constructed alongside constructions of queer sexuality. As we will see in the following chapter, HIV, queerness and PrEP are already linked in a dynamic system of ontologically open networks of matter and meaning. 


\section{Chapter 6 The Changing Constitution of the HIV Assemblage}

In the previous two chapters, we examined how PrEP was interpreted by queer guys. It appears from those discussions that low uptake of PrEP by queer guys is a result of both a problematic user configuration as well as difficulties with access. These problems are compounded by PrEP's uneven use in established prophylactic practices. Nonetheless, PrEP has the making of a revolutionary technology. While there is certainly a great deal of stigma around condomless queer sex, it appears from the accounts of my participants, that PrEP may further distinguish HIV-stigma from the risk of HIV transmission. Of course, how this may play out is highly contingent on how people respond to PrEP as it becomes more well known. What is certain is that PrEP enables new relationships that were not available before. Depending on which traditions a person holds dear and which relationships are deemed acceptable, these potential futures seem to be mixed. Indeed, as I discuss below, we can expect new forms of solidarity to emerge as a result of PrEP. In particular, users report feeling closer to their HIV-positive friends while non-users on the other hand seemed to imagine a different solidarity between them and HIV-positive persons. It appears that non-users continue to feel strongly that those who do not use condoms are immoral, ill-informed or thoughtless. This speaks to the larger technological frame in which PrEP is immersed.

I argue that PrEP, if employed in an accessible fashion alongside adequate information about the drug amongst queer guys in Canada, we may witness an ontological shift in the HIVassemblage. Both users and non-users sense that PrEP will be transformative in the ways we understand our relationship to HIV. Indeed, it is certainly the case that PrEP and HIV will reconstitute each-other in such a way that redefines the way sexuality is negotiated. Exactly how this will happen is hard to discern, as this future remains unclear. In order to understand the 
chances of PrEP's success, I consider how it is employed in relationships of co-forming social, historical and material relationships. I argue that HIV is inseparable from the technologies that attempt to make it intelligible; nor is HIV separable from the communities that engage with the virus, either through studying it, transmitting it, living with it or even imagining it. Indeed, the lasting effects of GRID become apparent in discussions of condom use, contagion and intimacy that speak to a "latent need" for PrEP. Whether that be allowing us to engage in a non-viral fraternity, allowing us to "clean" our blood or simply change how HIV is monitored in Canada, how PrEP is used continues to be multiple and certainly stands to change the constitution of the HIV assemblage.

\section{Changing Practices: Immunitary Solidarity and HIV-Risk}

Though PrEP is very much in its infancy, these effects are already evident in the narratives of my participants. Specifically, how my participants envisioned solidarity with HIV infected persons and ending stigma around HIV. Young, Flowers and McDaid (2016) note that proximity to HIV may be a factor in who ultimately decides to take PrEP, observing that "not living with HIV - or being close to those affected by HIV - shapes what information is accessible and who has access to information about PrEP." (418). While it is certainly the case that some users may already occupy social networks with a higher incidence of HIV than those of non-users, it appears that PrEP may reconfigure how HIV solidarity is enacted such that HIVnegative persons may no longer feel the need to distance themselves from HIV-positive persons. In other words, although one's desire to access PrEP may be negatively impacted by HIV stigma, if one manages to become a user there is some indication that PrEP helps mute that stigma enabling new forms of solidarity that transgress the boundaries of the sero-divide. 
This politics is already evident in how users and non-users envision solidarity with HIVpositive persons, PrEP users and themselves. As I discussed above, PrEP redraws the borders of immunity such that traditional methods of sexual hygiene and HIV prophylaxis become troubled. Not only does this seem to change how people choose to use condoms, but PrEP troubles practices of selectively choosing partners according to HIV status, otherwise known as serosorting. Evidently, PrEP affects practices of serosorting, as the practice is tied to knowing one's HIV status where status is a reliable indicator of HIV risk. As PrEP allows a person to fairly successfully guarantee that they are HIV-negative, and will be regardless of if they come into contact with HIV, PrEP users can engage in "bareback" sex without the risk of contracting HIV with both negative and positive partners. This means that PrEP users are able to cross the boundaries of positive and negative that are used to engage in contextual condom use, ignoring the mores that have circumscribed those communities. While some might argue that this allows a privileged few to ignore the traditions of queer sexuality, ultimately undermining queer solidarity, I argue that PrEP use may in fact create avenues for solidarity that were not available without PrEP.

The sero-divide that structures practices of serosorting are a direct result of the surveillance technologies developed in response to the HIV virus. The discovery of the HIV virus and the development of the HIV antibody test allowed for a conceptual shift from AIDS, a disease potentially affecting all gay men to HIV, a virus transmitted through particular practices and types of persons. This reconceptualization thus redefined the boundaries of risk from all gay men to specific kinds of gay men (Flowers, 2001, p.56). Bodies became the focus of HIV risk evaluations, specifically bodies "at risk" and bodies that "pose a risk" (ibid). The HIV antibody test thus conferred upon a person a status that informed how one related to their own body. These 
identity categories and practices of serosorting creates a system of "viral apartheid" (Dean, 2009 p.14) between HIV-positive and negative persons. Dean (2009) notes that one must juggle a desire to be inclusive of HIV-positive persons with the desire not to become infected themselves. Thus, it becomes necessary to the latex barrier of a condom to overcome the barrier of viral status. This exact point is not lost on non-users - 003 and I joked that PrEP seems to "tear down barriers," both in the literal and figurative sense.

The perception that PrEP will transform relations between persons with HIV and those who are currently uninfected was shared by both users and non-users. Both groups are optimistic about PrEP's ability to destigmatize HIV. Though, there are some slight differences in how PrEP users and non-users understand how that solidarity is enacted. This difference points to new potential fractures in the community and helps further clarify what characteristics might make the effective PrEP user. This fracture features divisions in understandings about solidarity with HIV-positive persons between PrEP users and non-users. While non-users more often spoke about HIV-positive persons distantly, users spoke as if they were closer to persons with HIV. Users seemed to see themselves as insiders with respect to HIV and expressed far more overlap between themselves and HIV-positive individuals than non-users.

Echoing Young, Flowers and McDaid (2016), my participants imagined the responsibility for transmission rested primarily on the shoulders of HIV-positive persons. However, contrary to the above study, my participants welcomed the possibility to take control of their HIV risk from their potentially serodiscordant partner. PrEP was perceived as a way for my participants to take on some of the responsibility for reducing HIV transmission. Consider the following discussion in focus group one: When I ask 003 to clarify what he means when he explains he is "optimistic about PrEP's role in ending stigma", 003 expresses that he knows there is a "lot of stigma about 
HIV-positive people" and he sees PrEP as a way to personally support persons with HIV. Struggling with exactly what he means, 004 and I suggest the word "solidarity." A term he finds acceptable. I ask 003 what he means by "supporting people who are HIV-positive." At this point 001 jumps in. He feels that PrEP lets you take on the responsibility for HIV status, so the person with HIV does not have to deal with the guilt on their own. When I ask who it is that has these feelings of guilt, 001 explains that PrEP would "balance out the responsibility". 001 notes that he appreciates the ability to "take responsibility" for HIV prevention, alleviating some of the guilt felt by HIV-positive partners.

In the second focus group, this discussion took the form of a change in attitudes toward sex, more generally. Non-users were excited at the prospect of having sex without a sense of anxiety. This sentiment is most clearly expressed by 008 who is excited at the prospect of a "magic pill" that will "allow sex to return." This sentiment is echoed by 006 who explains that PrEP demonstrates "evolution" in HIV prevention. He explains that before PrEP, people with HIV could not have sex and that PrEP is the first drug of its kind that allows this to happen, a point he expresses with optimism. This is echoed by 007 who notes that as PrEP becomes more accessible, it may help reduce the stigma "surrounding people with HIV-positive partners." This sentiment, that PrEP represents a substantial leap in mending relations between HIV-positive and HIV-negative persons, is shared by users. Christian, in particular, speaks passionately about his experience. His choice to become a user was largely framed in relation to his friends seroconverting. His narrative is particularly interesting, because his decision to take PrEP was framed not only as in his words "selfish" decision to allow himself to indulge in condomless sex. He writes that he "wanted to be a trailblazer and show that PrEP worked and that with this technique (medication), we could stop new infections" In addition to looking to 
protect himself, Christian began to learn about the social effects of HIV in the larger MSM community. As I noted in Chapter 4, it was his frequenting of the PrEP user online forums that rededicated him to becoming a user. Becoming part of the PrEP community, Christian became convinced of its effectiveness and decided that in addition to protecting himself he could personally work to reduce the stigma around HIV-positive persons. Christian describes his first day taking Truvada in a fervent tone:

My first day of taking Truvada for PrEP was emotional. I got dizzy. After decades of fear of HIV, of even associating gay with death, there was a path to overcoming the plague. It was all coming together. ART and PrEP were redefining what HIV management and prevention meant. We could conquer HIV and overcome our fear, shame and despair. There was a way to bring together poz [HIV-positive] and negative people.

He goes on to describe condomless sex on PrEP as liberating:

[Condomless sex is] like making a statement after the decades of trauma left by the HIV plague. There is also more intimacy; it feels better, physically, mentally and emotionally. The fear and stigma associated with HIV is fading. It creates a bridge bringing together [negative] and poz gay guys, after a long and shameful chasm within our community.

When asked about his positon on condomless sex, Christian writes,

It was liberating. Exhilarating. I got to fulfill a lot of my wildest sexual fantasies without any guilt or fear or shame. It got me closer to my poz bros, extending love, kindness and sexual attention in equal manner to them as to non poz guys. It made me HIV neutral.

Christian, here is demonstrating that his choice to take PrEP is both a personal choice, to stop himself from becoming infected, but in such a way that allows him to continue his relationships with HIV-positive persons. His argument though is that his PrEP use is as much a political statement as a personal one. He explains that after decades of being inhibited by the "HIV plague" he can engage in his sexuality without shame or fear of members of his own community.

Vince also notes that PrEP allowed him to sustain meaningful relationships with HIVpositive persons. At the time he started PrEP, Vince was single, so he started seeing a few partners casually, 
Some were HIV [negative] and some were HIV [positive]. But I actually had more comfort with the positive guys they were generally more sexually adventurous and less judgmental about my decision to bareback. I started a new relationship in September 2014 with an HIV [positive] man. 2 years later, and I am now married to him!

At another point in his questionnaire, Vince writes that he was far more anxious about his interactions with HIV-positive persons and sex in general in the past. Looking back, he regrets how restricted his sex life had to be, for fear of contracting HIV, But with PrEP, "the anxiety was lifted and I was able to carry out my sex life the way I wanted to. It's also thanks to PrEP that I started to date HIV [positive] men and met my husband." These narratives point to a significant change in the way HIV-negative persons who became users understood their relationship to HIV-positive persons once taking PrEP.

These stories point to a substantial change, a sense of liberation, experienced by persons taking PrEP. PrEP allowed HIV-negative persons to interact with HIV-positive persons in ways that persons were not able to before. Though both users and non-users explained that PrEP would enable new forms of solidarity, it is unclear if they have the same type of solidarity in mind. From how they spoke about themselves and HIV, users and non-users expressed diverging ideas about how they imagined HIV.

There was evidence that, with or without PrEP, stigma continued to drive a divide between those not taking PrEP and persons with HIV. When speaking about HIV or HIV infected persons, non-users nearly always spoke in such a way that they distanced HIV-positive persons or spoke about their relationship to HIV in the second person e.g. "you can do something before actually contracting HIV" (002), or "There is a definite stigma with admitting to people especially like family that you may have sexual relations with a person who is HIV-positive and like you could get in trouble with your family for that..."(001). This emotional distancing points to two interesting themes, one, that PrEP non-users affectively distance themselves from HIV 
and two: that they, for all intents and purposes experience HIV as distant from themselves. This is a key difference that speaks to an interesting contradiction in how users and non-user position themselves as at risk of HIV. Echoing Young, Flowers and McDaid (2016), HIV-negative users are intimately aware of their HIV risk, running in circles with HIV-positive partners. Examples include Vince and Christian, who speak about the times they interact with the HIV-positive community as just another one of the many groups with which they interact. Users further speak fairly candidly about their HIV risk, for example, noting that “... and in the back of my mind, I felt I may end up becoming HIV [positive] one day.” (Vince) or as Eric writes, “... I would never have to tell my Mom I'd contracted HIV. Too many times had I 'slipped up' and been driven by hormones to bareback sex, I eventually asked myself 'How many times can you say, 'never again?'”

The contact between users and HIV-positive persons is a significant difference between users like Vince and Christian and non-users. Though interested in PrEP's role in destigmatizing HIV-positive persons, non-users did not mention their connections with HIV-positive persons. Non-users, rather, chose largely to speak about HIV-positive persons distantly, as if they were not in contact with persons with HIV. As Doucet and Mauthner (2008) note, these differences in subject positioning "highlights where the respondent might be emotionally or intellectually struggling to say something and/or signals varied meanings in the respondent's perceptions of self" (408). This is interesting, not only because it points to rifts in solidarity between users and non-users, it points to a potential for increased risk in the non-user group. Harvey (2011) notes that HIV is often remembered "by placing oneself at a distance from its threat" (172), noting that HIV is effectively forgotten by the barebacker by abandoning the self to it, by bringing it closer to them. In this sense, "accepting one's risk" as Vince puts it, allowed him to see himself as a 
part of the "poz" community. This is an interesting dynamic the logic of which, without PrEP, would seem to correspond risk with behaviour. What is key here, however, is that the affordances of PrEP allow users to overcome that forgetting by allowing HIV to enter their bodies, without the risk of infection.

Drugs are a "catalyst" through which new socialites appear and transform (Collins, 2016). It is increasingly clear that the implementation of a PrEP regime will change relations around HIV. Poicus (2016) notes that PrEP enables a dissolution of this form of solidarity or "viral fraternity" sustained by barebacking and facilitates a shift "towards a neoliberal sexual individualism in which access to chemoprophylaxis enables immunity, both in terms of biological immunity and in the classical sense of the term as an exclusion from the obligations of the polity." (32) While this certainly remains a possibility, from my participant's discussion it seems that PrEP represents a far more pervasive shift than outlined across an infected/noninfected binary. It seems that PrEP facilitates novel forms of solidarity that are not premised on notions of contagion. Collins (2016) argues that, when theorizing the social role of pharmaceuticals, it is imperative to understand that "the same molecules have different end uses (and even effects) depending on the context in which they are consumed" (84). The multiple interpretations of PrEP by Poicus (2016), and the diverging attitudes made by my participants, point to emergent forms of novel sociality.

What we see from this discussion is a changing dynamic, a melting of previously stable flows of social behaviour and a recrystallization of the relations that were the positive and negative/unknown status groups along new lines. It also points to the resistance of some of those groups to this redefinition. My non-users were unable or unwilling to see themselves on the other side of the HIV-status divide. Of course, it remains unclear whether or not this self-ideation will 
determine uptake of PrEP, or if it is a result of taking PrEP itself that causes a person to relax their guard with respect to HIV-positive persons. What is important to note however, is the lingering relationship between HIV-positive status as a stigmatized identity and the extension of that stigma into health care materials or preventative treatment (Payton \& Kvasny, 2016). What is definitive however is that overcoming this stigma continues to be a significant barrier to PrEP's success as a preventative technology.

\section{Changing Practices: PrEP use and Obtaining Consent}

PrEP stands to reconfigure social relations along previously established lines of HIV risk. Specifically, it may redefine the notions of contagion that inform calculations of HIV risk. On one hand, for some, PrEP represents a divorce of HIV from barebacking practices, allowing some to engage in otherwise "risky" practices without fear of contracting the virus. For others, it appears that the purpose of PrEP is to protect them from persons deemed at risk. These parallel uses for PrEP point further to emergent divisions facilitated by this novel prophylactic. There is a possibility that some of these groups might only seldom interact or avoid each-other altogether, forming discreet and divergent communities with divergent risk profiles. What is clear from my participant's discussions is that despite identifying as members of similar communities and outlining similar sexual practices, participants interact in different socio-sexual circles with very different sexual mores.

Running parallel to the theme of solidarity between queer persons is a sense that PrEP can protect a user from harm, by keeping themselves at a distance from "risky persons." Especially if the person with which they were engaged was deemed untrustworthy or "dirty". This theme came nearly exclusively from non-users. 008, for example, notes that PrEP would be useful to protect himself against an unsavoury sexual partner. He explains that PrEP would be 
useful for him after "a one-night-stand with someone you felt was a bit skanky," explaining that the pill would be useful assuaging anxieties about that person. In this instance, his encounter is not framed in terms of HIV infection, but in terms of his relationship with his partner. He explains that, after a sexual encounter,

you're not focusing on the war the pill is having in your body, you're thinking about the context of what you got yourself in...I mean if you did or had unprotected sex with someone for just a one-night stand and then you take this pill the next day you might feel a little bit easier about what just happened rather than as you explain feeling as if there was some potential HIV war going on in your body.

The difference between 008 and the accounts of persons like Christian or Vince in the previous section is dramatic, pointing to different attitudes about the "type" of person who contracts HIV. What is evident here is that 008 and these users' interpretation of PrEP is diverging, one as a way to bring together persons with HIV and those without, and on the other, as a way to protect yourself from them that is more in line with points made by Poicus (2016). This point is not lost on 007 who notes that, in response to 008 , the fear that you have HIV replicating in your body may have more to do with an "internal fear" associated with the stigmatization of HIV rather than anything else.

In addition to evidence suggesting that a large-scale implementation of PrEP may lead to divergences in sexuality across Ontario, there is some indication that PrEP may generate two new social groups divided according to prophylactic preference with distinct risk mitigation practices. There is already evidence that queer guys who engage regularly with HIV-positive persons are more likely to comprehend their individual risk and discuss HIV status openly with their peers. In a study of HIV-negative gay men, Keogh (2008) found that perceived proximity to HIV-positive persons was related to impressions of HIV. These "low proximity" guys download the responsibility to disclose on HIV-positive persons, who as Adam et al. (2008) argue rely on 
circuits of cultural capital to determine when to disclose, what constitutes disclosure and how one discloses. Further, HIV-negative men who report little contact with HIV-positive persons held overall more negative perceptions of HIV in general, perceptions that Keogh (2008) argued may not be conducive to status discussions at all. Persons who perceived themselves as more proximal to HIV-positive persons were more likely to experience HIV as a part of every-day life, where disclosure was commonplace and acceptable. Keogh (2008) concludes that HIV risk reduction should be targeted to the low contact group, as they appear to "use a concept of responsibility which does not have the capacity to deal with" (Keogh, 2008, p.580) the complexity of negotiating safer sex. Indeed, these effects appear to be compounded for HIVnegative PrEP non-users as from some discussions it appeared that the criteria for an "unsavoury sexual partner" might extend as far as to encapsulate all PrEP users. Themes emerged in my focus groups that PrEP users are not to be trusted or that PrEP users are somehow violating the bodies of their partners simply by not wearing a condom.

Consider the contrasting constructions of a PrEP user between 002 and 004. Both 002 and 004 are very clear that they prefer condoms as their method of HIV prophylaxis, though they are interested in PrEP as a safeguard against condom failure. 002 is clear that he is not interested in having sex without a condom, even if his partner was on PrEP. He notes that if a person were to inform him that they did not want to use a condom, he would reply "okay cool, then we are not having sex." He cites concerns about other STIs as one reason why he would not forego condoms despite the person telling him they are on PrEP. 004 says that "if someone tells me they aren't going to have sex with me if we don't not use a condom if they are pressuring me that way then I'm going to say, 'fuck you and no."' They continue, that if someone were to say they want to have sex without a condom it does not seem "very consensual" to them. 004 feels that whether 
or not a user would consider wearing a condom as a good test of the character of the person. 004's points are illuminating to the conflation of a person's character with their condom use. That simply the implication of not wearing a condom is enough to make 004 so uncomfortable as to begin constructing a sexual assault scenario in their mind.

Though the sense that PrEP users violated the bodies of non-users was not a significant theme in focus group two, these participants too eventually constructed a scenario where the PrEP user was unsavoury. In this instance, participants discussed the possibility that the alleged PrEP user may be lying simply to get consent and/or to forgo using condoms. 006 does not think he would have sex with a person who told him he was on PrEP. How can he be certain that his partner is telling the truth, he asks? He says, "I feel some people might be lying." I ask 006 why he thinks people would be lying about being on PrEP? He replies that maybe this partner really likes him or perhaps he just wants to have sex and does not want to "hear no from you."

In response to this discussion, I ask if people think saying that you are on PrEP might be a way to "grease the wheels," that is to say make the encounter go a bit more smoothly. In response participants began to construct a user who was trying to demonstrate their class position or at least their disposable income. 005 quips, "I've got a fancy car, I've got PrEP." 008 notes that he supposes he would likely perceive a person who is taking PrEP to be more responsible than others, something some non-users might capitalize on not to wear a condom. These discussions point to the complex interplay of symbols and taken for granted rules that go into a consensual sexual interaction (Adam et al., 2008; Dean, 2009). Indeed, James mentions that there are a "surprising" number of people in Toronto who are lying about being on PrEP. James supposes that this is because PrEP is a "social or financial construct (perhaps people feel they are perceived as poor or unsafe if they are not on PrEP.)," that people can derive some positive 
social value from identifying as a PrEP user. To make sure people are actually on PrEP, a friend told James and (presumably) his partner to make sure they see them take the drug. Otherwise they might be lying.

It appears that the visual confirmation that the sex was "safe" was necessary to both users and non-users in negotiating consent. For example, when I ask members of focus group two what they would do if a partner told them they did not need to use a condom because they were on PrEP participants were highly skeptical.

[005] But how will you know he's on PrEP?

[Adam] 'Cause he's told you

[006] But like I feel like *unintelligible*

[005] Maybe they're lying, I don't know.

[006] *laughs* Yeah.

[Adam] So you're like...

[005-] Maybe just show me the bottle.

At this point the rest of the group erupts into laughter and 007 jokes, "I want to see your Truvada prescription." 005 is serious though. What if the partner is lying? He is worried that he might be duped into not using a condom. He explains, "I'm [not wearing a condom] and then next thing you know you end up knowing that you don't really do anything." 008 explains that this underscores that it is up to the non-user to determine whether the user partner is in fact telling the truth or not. He explains, "you are being a consenting trusting adult with this person ... if you think he's going to lie to you already then maybe you shouldn't be in that situation to begin with.” Echoing this statement, 006 explains that if he cannot see the bottle than he would wear condoms before doubling back and saying that even if the user was able to provide proof that 
they were on PrEP they would still use condoms. It is clear that the visual confirmation that a condom was being used stood in for a portion of consent for some of my participants. Without further clarification of who this partner was and no indication that they could trust this partner, a condom was deemed necessary.

These discussions further clarify attitudes held by non-users that point to further divergence. It appears that, to non-users, there may even be a latent sense that barebacking is inherently immoral. I ask the first focus group if they think of barebacking as part of the bug chasing subculture. 001 notes that he is not aware of a bug chasing subculture and denies that this is the case for most persons who forego condoms, stressing that persons who go without condoms are simply misinformed or reckless. He argues that barebacking comes down, to both partners being recently tested and seeing each other's test results and being assured about that. But, like, I don't think that people who are less stringent about that are actively searching for HIV — but I think it's more of like testing. They're not understanding exactly how risky it is or not caring because of a lack of education or care for themselves.

001s comments point to a significant barrier to the success of PrEP as a preventative technology. The conflict between existing frames of knowledge about HIV and safer sex as they rub up against PrEP as a technology. Bijker $(2010 ; 1987)$ defines a technological frame as structuring the interactions around an artefact. In this case the artefact is HIV, and the technological frame is the problem-solving techniques used by those at risk of contracting the virus. Bjiker (1987) further defines a technological frame to include elements such as "current theories, goals, problem-solving strategies, and practices of use" (171-2). For better or for worse, HIV has been constructed as a lifestyle virus (Treichler, 1987) that is both the result of particular sexual practices and biological processes. That is to say, HIV has been simultaneously constructed as a gay disease, as well as a disease that is spread through unprotected anal sex, among other routes. 
As a result of this frame, health messaging has both targeted gay men and stressed condom use for anyone regardless of sexual orientation (Epstein, 1996). However, this frame has generated stigma for persons who do not practice "safer sex." As 002 notes, "all health messaging geared to queer men" has stressed condom use, which has led to some stigmatization of persons who bareback. This messaging is that it is a shared obligation of all queer persons to address HIV through safer sex practices (Yep et al., 2002). In this sense, both barebacking and PrEP are seen as an affront to the collective, leading to a stigmatization of barebackers as irresponsible (ibid) and PrEP users as "Truvada whores." This "sex positive" method of safer sex developed as an early response to the HIV epidemic (Epstein, 1996) now stands as a serious barrier to PrEP's potential as an effective HIV prevention method.

Participants in focus group one echoed participants' responses to the first scenario in the second focus group. Where non-users are asked to imagine a casual encounter with a PrEP user, non-users were not interested in foregoing condoms. Emerging from this conversation was a serious concern that PrEP does not protect against STIs other than HIV. I ask how they feel about the assertion that some users note that they take PrEP because HIV it is the only sexually transmitted infection without a cure (a justification made by PrEP user Mark). 002 and 003 were noticeably shocked by this idea. Though after a moment, 003 points out that though getting an STI is unpleasant, he "knows guys who think this way." He says that this is their personal choice but they are not the kind of person he would want to have sex with. Agreeing with 003, 002 says that he "judges" this person. He personally feels strongly about STIs as he hates getting sick in any way whatsoever, an anxiety that is compounded by the reality that STIs are stigmatized. Reflecting on his qualms with STIs, he feels strong negative feelings about STIs, but he is not sure why. Even though he understands that STIs are common and not objectively any worse than 
any other disease, he feels that his "brain puts STIs in a horrible category." He understands STIs as a normal "every day" thing, but when he thinks of them he is disgusted. In response to 001 , 003 says he is a bit more pragmatic about negotiating condom use. He notes that if there are no condoms available, he proceeds to serosort - that is to say he asks when they were last tested and depending on their status and test date, he decides whether or not they should forego the condoms. For 004, however, condom use is a question of consent. They point out that if they wanted to wear a condom, then if this person does not provide a condom and presses him not to use one, then they are not going to have sex. 004 does not "tolerate that sort of thing."

While non-users in my focus groups seemed hard pressed to imagine why people would bareback, users note that they have no trouble finding people who actively want to forego using condoms. Interestingly enough, Vince writes that he is aware of a "very large segment of the population, particularly men (gay or straight), [who] craves raw condom-free sex." He supposes that "a lot of HIV [negative] men are desperate" to have condomless sex and see Vince as a safe way to do so. Vince attributes this need and his propensity to engage in condomless sex as the reason he has been able to have such a healthy sex life. In other words: barebacking made him sexy. Now that PrEP has made condomless sex more common, Vince notes that he is less "in demand" than he was a few years ago. This discussion echoes user Christian who notes PrEP meets a "latent need" in the gay community as well as Eric's surprise at how open his HIVnegative partners were to condomless sex (though he notes that maybe his partners were "same types of guys who are naïve enough to believe anyone who says they're negative really is negative.")

These discussions point to a clear divergence between my sample of PrEP users and nonusers. This divergence may reflect the same one between PrEP users and the peers they feel 
"judge" them. What is particularly interesting about this, however, is the conflation of safer sex practices developed in response to HIV with HIV stigma. For example, when James discusses PrEP with one of his friends, he notes that his friend is critical of PrEP's effectiveness and encourages James to continue using condoms. However, James notes that this friend does not get tested regularly, while he gets tested regularly now that he is on PrEP. He notes that he finds it interesting "that other gays would slut-shame and judge when they themselves do not know their status or have not been tested for an STI." He continues, noting that he feels,

there is still a lot of slut shaming and self-hating within the gay community. People would rather not know their status out of fear of being perceived as sexually active or being a slut. When I was younger I cared what other people think. Now that I actually feel like I am part of a community and have made many like-minded friends I no longer care.

We can interpret James' comments as reflective of the paradoxical nature of PrEP as it interacts with 'condom only' HIV prevention discourse. As PrEP users acknowledge that they will fail to use condoms, they confront their partner as both at risk and a non-risk: a non-habitual risk taker. On one hand, this form of drugged sexuality allows, for the first time, for a user to disconnect their desire to not wear condoms from the notion that they are willfully self-destructive (Race, 2016). However, at the same time, it is impossible not to notice the outcry of disapproval from HIV organizations, media outlets and in online discussions that PrEP users are simply "Truvada whores".

Thus, while PrEP may reconfigure solidarity for queer persons, it is emerging within a larger technological frame and corresponding classificatory system around HIV. Common understandings of contagion and risk will certainly bear on the success of PrEP as either a useful intervention for all queer guys or as a sexual enhancement drug for rich queer guys who want to eschew established methods of sexual hygiene. If PrEP's relationship to these established 
prophylactic methods are not fleshed out for members of these communities, we might see further divergence between the condom only camps and the barebacking communities. These emergent groups will need to be targeted for HIV prevention efforts differently.

\section{The HIV Assemblage: Constructing Queer Identities}

Evidently, PrEP is received unevenly by my participants. Non-users in particular express contradictory ideas about PrEP. On one hand, they desire the drug, as it would ease their minds and help them download some of the responsibility for HIV transmission from their potentially positive partner to themselves. On the other hand, non-user participants seemed not to trust their PrEP-taking hypothetical partner. Users, on the other hand, express the experience of being able to overcome the sero-status divide with enthusiasm, while nonetheless experiencing condemnation from their non-user friends. In order to understand why this drug is experienced as an unwelcome blessing, I would like to return to how PrEP is agential through its affordances. As outlined above, these divergences are along the lines of prophylactic preference which means that prophylactic preference is related to how participants understand their relationship to HIV. Evidently, a person's prophylactic preference impacts their understanding of HIV risk and contagion more generally.

The following discussion outlines how HIV prophylaxis and safer sexual practices emerged in a network of historicized relationships. Relationships that emerged in response to the initial outbreak and then quickly mutated as those prophylactic practices changed how the disease was spread. Most importantly, I discuss how these prophylactic practices are related to notions of contamination, contagion and intimacy. I argue that PrEP may be seen as both a curse and a boon in part because it interacts unevenly with how queer people have adapted to avoid contracting HIV. The construction of HIV as a queer illness, and the presumption of toxicity is 
reinforced by the barrier erected by condoms and challenged by PrEP as it allows a person to transgress the barriers of HIV-status. These affordances are interesting and worth considering as it speaks to alternative uses for PrEP that may not have been apparent at the design stage. Specifically, that PrEP enables an undoing of contradictory notions of intimacy and contamination that, at least from the narratives of my participants, have contributed to condom non-adherence. If this is indeed the case there is significant reason to be optimistic about PrEP's ability to significantly impact the role of HIV in Canada, should it be implemented properly.

Queer as an organizing concept for my community is interesting due to its double meaning. The queer body is historically an unwell body (Halprin, 2016). It was not too long ago that to feel 'queer' meant to be unwell (Chen, 2012, p.98). To be queer was to be contaminated, a social contaminant if nothing else. Queer persons, historically have been seen as both mentally unwell and a toxin in and of itself to the social body. Of course, queer bodies can also become unwell; they can become drugged or can become unwell due to a physical or mental illness. For some, intoxication is key to an affective future (Munoz, 2008), for others it represents a debilitation (Chen, 2012). Though the so-called sexual revolution of mid- $20^{\text {th }}$ century was poised to de-stigmatize queer sex. Indeed, the vote to remove same sex attraction from the DSM in 1973 was a substantial step toward the de-pathologization of non-heteronormative sexuality. However, the discovery of AIDS in gay men and the cementing of queerness in HIV surveillance undid much of those strides as queer sex was no longer necessarily a mental illness, but was reimagined as a pathway for a highly fatal illness.

The incidence and distribution of HIV has evolved significantly over the last 40 years. Initially AIDS was a "puzzling" trend. An unexplained increasing prevalence of opportunistic infections in homosexual men. This "disease that plagued gays" quickly came to be known as 
gay-related immune deficiency or "GRID” (Epstein, 1996). Not shortly after, this condition quickly expanded into communities of heroin users, homosexuals, Haitians and hemophiliacs (Epstein, 1996; Treichler, 1987). GRID was formally reclassified as AIDS to reflect these expanded areas of incidence. These categories shifted as the virus, HIV, was discovered and became better understood as the underlying cause of AIDS. Understanding that HIV caused AIDS allowed health researchers and care providers to further clarify transmission pathways, shifting the focus from types of people at risk for HIV to the types of practices that put one at risk for HIV (Flowers, 2001). These shifts are reflected in Health Canada's most current typology of HIV risk groups. As of 2014, Health Canada uses the following "exposure categories" to monitor HIV transmission: "MSM," injection drug user, transmission from blood or blood product, heterosexual from an endemic country, heterosexual contact with a person at risk and heterosexual contact with no identified risk (Public Health Agency of Canada, 2014). This typology, though mainly focused on risk behaviours does retain qualities of earlier group oriented risk evaluations.

These routes of transmission and corresponding social groups have changed significantly over the epidemic. Though HIV is arguably no longer a "gay disease," it continues to disproportionally impact MSM (Public Health Agency of Canada, 2014). Of the 66\% of HIV transmission cases where status group was known, MSM transmission accounted for $49 \%$ of transmissions. Though MSM occupy the lion's share of novel HIV diagnoses, heterosexual transmission is not far behind. Heterosexual transmission of HIV accounts for $30 \%$ of novel HIV diagnoses in 2011 . The remaining $31 \%$ of transmissions are more or less evenly distributed amongst the other exposure groups (Public Health Agency of Canada, 2014). In another report, we can begin to see the fragmenting of these trends in HIV transmission across regions in 
Canada. For example, the Public Health Agency of Canada (2014) reports that though MSM remain the major vector of HIV transmission in Eastern Canada, HIV is increasingly endemic in heterosexual and injection drug using communities in the prairies and in $\mathrm{BC}$.

PrEP is emerging in a shifting landscape of HIV transmission. The widespread use of PrEP and these changing dynamics of HIV transmission mean that HIV can no longer be reliably monitored according to these exposure categories. As Flowers (2001) has noted, each technological development in response to HIV has served to reconfigure HIV risk. This reconfiguration of HIV risk has, generated a system of inequalities based both on one's historical relationship to the disease and on one's proximity to the disease (Dean, 2009; Keogh, 2008; Young, Flowers \& Mcdaid, 2016; Race, 2016). As we have discussed, PrEP, should it become a mainstay of HIV prevention, will most likely enable a pivotal shift in HIV risk evaluations. Though "heterosexual" was certainly not one of the four Hs of HIV transmission in 1984, the widespread targeted use of PrEP in MSM communities stands to increase heterosexual representation in HIV transmission statistics. As prevention strategies shift from the "molar" to the "molecular" level (Rose, 2007) it is increasingly difficult to continue to monitor HIV transmission according to behavioral or identity categories.

Clearly, PrEP is emerging unevenly in a network of relationships that have been framed by over 40 years of HIV response. PrEP is engaging with the lasting effects of each of the ontological shifts experienced by the HIV assemblage over the last 40 years on our current understanding of HIV risk, as it extends into and beyond this target community. In order to do so it is key to imagine HIV and its constellations as dynamic and co-forming interactions of the social, historical and the material. It is perhaps generative to imagine these movements through what Tuana (2008) calls the "viscous porosity" of nature/culture. That is to say that HIV is 
inseparable from the technologies that monitor and graph it; nor is HIV separable from the communities that engage with the virus, either through studying it, transmitting it, living with it or even imagining it. It is useful to imagine the HIV assemblage conceptually as viscous and porous; as attending to the permeability of interactions using this organizing concept

helps to undermine the notion that distinctions, as important as they might be in particular contexts, signify a natural or boundary, a natural kind. At the same time, viscosity retains an emphasis on resistance to changing form, thereby a more helpful image than "fluidity," which is too likely to promote a notion of open possibilities and to overlook sites of resistance and opposition or attention to the complex ways in which material agency is often involved in interactions, including, but not limited to, human agency. (Tuana, 2008, p.194)

PrEP certainly draws our attention to the porous quality of HIV risk, as well as the syrupy nature of risk categories. This is key, as the dual nature of HIV as a gay community disease, on one hand, and as a disease related to risk practices on the other, informs how we understand our selves. This careful dance between the body, nature and the social is not lost on Rose (2007) and Rose and Novas (2005), who argue that although medicine has always had a role in defining what it means to be human, it is perhaps the case now more than ever that we enact our citizenship through our biology. This engagement with virus and virus-related risk is central to how my participants discussed their desire to use PrEP

HIV cements the dynamics of sexuality along predictable lines. One of the reasons PrEP has been so controversial is because it generates new ways of being that confound this sexual schema (Speildenner, 2016; Race, 2016). As Pocius (2016) notes, the logic of immunity that defines HIV is broken apart by PrEP. As I explained above, I do not think this conceptualization comprehends the relations enabled by PrEP in their entirety. Pocius is useful, however, to the extent that he allows us to understand quite clearly the shifting dynamic of safer sex practices in light of the historical communities that these distinctions have served. 
Rather than understand PrEP as exclusively defined by the communities that use it, I have attended to the relational quality of PrEP as it interacts with HIV and "at risk" communities. Doing so allows us to understand the malleability of the communities and their technologies as they interact over time. Collins (2016) argues that it is necessary to think through pharmaceuticals in this way. Pharmaceuticals play a significant role in generating collective identities, not just by serving as a referent through which people understand themselves as alike but by changing the body itself. These changes allow for people to inhabit novel social locations that may have been unavailable to them before. To return to Tuana (2008), the HIV assemblage is viscous, but nonetheless ever changing. Persons and artefacts seem fixed in roles, but those roles shift nonetheless. The HIV assemblage is also porous; these distinctions between at-risk and not-at-risk are not grounded in a universal nature but are semi-permeable boundaries through which certain actors osmose. PrEP makes this painfully clear: for those who can overcome its barriers, HIV is rendered harmless. Nonetheless, these relationships; of person to virus, drug to virus, person to drug and person to person, are all necessary in understanding how a person lives and dies.

Rosengarten and Michael (2010) argue that PrEP should be thought of as a "prophylactic 'assemblage"" in the sense that it is not simply a pill "but a complex of heterogeneous elements that emerge through their relations." This assemblage has offered a subjectivity that creates a sharp distinction between queer and not queer, where queer sexuality is seen to be at risk, leaving socially sanctioned forms of sexuality in a zone of viral exclusion. Though the reality of this zone of viral exclusion in practice is certainly debatable as it is itself racialized and confined largely to the global south (Porter \& Kaplan, 2011; Poicus, 2016). As we can see from the 
following discussion, PrEP brings to the fore new possibilities for persons to thrive, it also brings to our attention the ways that citizenship is increasingly understood in biological terms.

The role of PrEP in enabling new ways of life is best illustrated in a conversation between myself, 003 and 001 , in my first focus group. In discussing his hopes with respect to PrEP, 003 expresses his excitement to give blood. This statement initially struck me as rather odd because PrEP has nothing to do with blood donation, nor would it bear on Canadian Blood Services blood screening procedures. Surprised, I delve into 003's comment for clarity. His response defies paraphrasing:

Yah. I mean, like, I give blood. I don't give blood. I have blood tests very frequently, but I can't give blood because of my HIV risk. So, I guess. In my life, I guess-- like my father, it was really hard coming out to him and I dealt with a lot of his ignorance about HIV for the first couple years and it was really hard for me to talk to him about my sexuality. Like, he grew up during the HIV/AIDS epidemic so he has a certain idea of the type of people that get AIDS.

Pressing 003 for further explanation was just about as perplexing as his initial point. His ideas about of HIV, medical care, risk and his relationship to his father after coming out as gay are all wrapped up in his blood. For him, all these notions were related to PrEP. PrEP represented a "positive trend" for persons discriminated against in medical care. He gives blood frequently for a number of other reasons, yet is unable to overcome a barrier that is in his very blood. 003 's inability to divorce his blood from blood tests, his experience of medical care, notions of responsibility, his relationship to his father, his sexuality and HIV/AIDS resonates with Puar's (2008) assertion that queer identity is a dynamic system that cannot be dissolved into a "temporal, spatial, energetic, or molecular distinction between a discrete biological body and technology" (217). 003s blood is indistinguishable from all the above. We can then understand how PrEP can reanimate parts of 003s life that have been killed by the conflation of HIV and his sexuality. 
Though I was surprised, my participants seemed to follow 003's point as it followed an as yet unclear contextual logic. This contextual logic when further clarified, unwrapped a network of relationships between blood, toxicity and unlife. What is particularly interesting, however, is exactly how far these relationships went. They transgressed personal, inter-group and national boundaries. Both 003 and 001 demonstrate how the presumption of toxicity enabled this social death on one hand, but also translated into real loss of human life in another. When pressed, 003 asked me how it feels not to be able to donate my own organs because of my sexuality. While attempting to answer his question, 001 interjects with the intent of clarifying 003's point. 001 notes the role of blood in the recent massacre in Orlando as an example of how blood was related to PrEP. He explains that,

After the whole [Pulse nightclub] shooting they were calling for blood donation from people in the Orlando area it was just so-- you know-- half of the people actually affected by the shooting could not give blood to like their loved ones and stuff.... I don't know, it's cruel to be kept out of like helping other people especially other people who are being harmed for the same reason that we aren't allowed to help them.

001's comments complement 003's, further clarifying the relationship between blood, HIV risk and the emancipatory potential of PrEP. Due to the presumption that our blood is in itself a harm, actual lives were lost. Responding to 001 's point, 003 further expands this point connecting medical care, Orlando, intimacy and PrEP through his discussion of barriers:

I just think that queer people have barriers like socially and like in relationships. That say ten or twenty years ago you entered a relationship with someone who was HIV-positive but you weren't able to be intimate with them for fear of contracting HIV ... I just think that situations like that are unfair and seeing PrEP as a potential way to avoid situations like that... I'm fully supportive of it.

In light of these comments my participants' discussion of condoms, intimacy, blood and selfimage and the decision to use PrEP is itself a revolutionary act. Non-users and users alike seem to be aware of the possibility and indeed see new futures as a result of a wide spread 
implementation of PrEP. It is clear that my participants are not interested in PrEP because they are bug chasers, which would defeat the purpose of using a prophylactic. Nor, as we have noted, are many of them even necessarily exclusively barebackers looking for a way to engage in condom free "disinhibited" sex. Finally, it does not seem that PrEP is drawn along the lines of immunity in the sense that those who use PrEP represent a privileged few entering the heterosexual mainstream. What is brought to light by PrEP is exactly how life in the shadow of GRID bears on my participants, conferring a sense of intoxication on all queer men universally. PrEP thus is redefining immunity, however in a far more pervasive fashion than some have suggested.

\section{Revisiting the "Latent Need" for PrEP: A Political Promise}

Users noted that PrEP met a "latent" need in the queer community. A need that extends beyond HIV risk as defined by its designers. This latent need has to do with notions of contagion attached to queer bodies. Specifically, that HIV risk is conflated with queer identities such that queer bodies are presumed toxic with or without an infection. These are the key vectors of PrEP's interaction with the HIV assemblage. In the following section I will further examine exactly how PrEP may destabilize these concepts of toxicity. We will revisit the question of disinhibition and imagine how the conflicting logics of intimacy and HIV risk, identified in are reconfigured by PrEP.

To be dirty, toxic or contaminated can be framed according to nation, community (Wald, 2008), or based on a personalized risk portfolio (Hacking, 2003). Indeed, Gilead Sciences has sponsored an informational video that constructs PrEP as active at each of these levels (Amico et al., 2014). This video, hosted on a website entitled "What is PrEP," portrays PrEP as creating a steely barrier around both the cell and the body, in a nation whose map is pockmarked by HIV 
transmissions. Though for most people, this video is unproblematic, it is certainly not false in its description of how PrEP functions. This video is, however, a bit deceptive as it misrepresents how PrEP interacts with HIV. PrEP's actual mode of action does not erect any form of barrier. Unlike condoms, PrEP does not shield the cell nor your body from infection. It's mechanism of action only occurs once a cell is already infected. In this sense, you are not so much protected from infection as you are unable to transmit HIV to your other cells, let al.one someone else.

This imagery is important because it speaks to the way we engage with HIV as part of the HIV assemblage. One where HIV is seen as a more or less harmless agent, rendered inert by PrEP and another where HIV poses a threat to cells, bodies and the nation as a whole. I outlined above two contrasting styles of thought emergent with respect to HIV that parallel these competing ideas. One whose experience of HIV was one of HIV neutrality, and another who was interested in using PrEP to shield themselves from HIV. Poicus (2016) notes that this logic of immunity allows gay men to engage in barebacking without the notions of viral fraternity associated with prior notions of barebacking. Though what seems to be emergent is a sense of fraternity without the virus. It seems to be that this is because HIV and queer bodies are conceptually fused as one in the same, regardless of the virus. What happens to notions of contamination when we imagine the body, not as shielded, but where the virus is rendered inert.

Chen (2012) defines a toxin or contaminant as two proximate "bodies," whole or in part, living or abstract, where one body poses a threat to another. The toxic body has the effect of altering or effecting a damage to the other body, to the point of disability or death (191). This sense of toxicity, Chen argues, is wrapped in the "fabric of immunity nationalism" that extends beyond subjects that are traditionally cast as biopolitical. In this sense HIV, PrEP and queer men are all more or less equal biopolitical subjects interacting within the same assemblage. In a 
similar vein, Braidotti (2009) suggests framing the biopolitical relationship as a relationship between human and animal life, as opposed to the relationship of the sovereign to subject. Braidotti (2010) rather than conceive of zoë as "that in you that the sovereign can kill," to use the concept to think through non-humans and humans in such a way that brings the human and nonhuman together in ways that are generative, averse to pessimism and that avoids the notion of death as the antithesis to life. Using this theory to analyze the shifting notion of "contaminant" or "toxin" can help us understand how PrEP serves to change HIV-risk and queer subjectivities as a result.

Condoms and PrEP operate very differently ${ }^{14}$. Because their method of prophylaxis is so different, it is worth considering how they engage with an HIV infected body. Consider the construction of the toxins we have considered until now. Our first toxin, HIV, like all viruses, is constructed as an outsider. The metaphor of outsider or immigrant status is often conferred upon viruses as the horde at the gates (Wald, 2008; Martin, 1994). Using condoms as a mode of prophylaxis reinforces this sense that HIV infection occurs once a virion "breaches our defenses." Constructing the HIV-positive body as part of that hoard at the gates, however, is problematic, as we will see, for intimacy, especially as we have already discussed, when we are socialized through prevention efforts to assume all queer bodies are potentially infected.

In other instances, viruses are conceived of as an undead creature, neither quite alive or quite dead. This is not unlike the construction of gay men post-outbreak. In many ways discussions of gay men are unavoidably framed through not-quite-humanness in light of HIV infection. Invoking the metaphor of the ghost, Muñoz (2009) notes that gay men haunt this world, longing, waiting for a world without HIV. Ghosts in this sense both imply exclusion and

\footnotetext{
${ }^{14}$ I want to make it very clear that I am making this argument, not to discourage condom use, but simply to point out that the way PrEP and condoms operate enable different subjectivities.
} 
inferiority (Muñoz, 2009, p.42) and much like the virus itself, existence in the shadow of HIV is neither quite alive nor quite dead. This form of unlife for Munoz is generative, however, as it creates horizons of possibility for queer futurity. For Chen (2012), however, deamination or unlife is more problematic. Though the point is well taken, intoxication as a form of Jouissance can be transgressive in its own right. Nonetheless, I see a lot more of Chen than I do of Muñoz in my participant's thoughts on contamination. I cannot help but liken my participants experience with Chen's own experience, living through intoxicated interactions with their partner/lover. Chen's discussion of the arrested state of being, waiting until the toxicity passes and they can be themselves again is painfully similar to my own experience in my community as a whole. I argue that Chen's discussion of the role of toxicity in intimacy can help us further understand how PrEP serves needs beyond those identified in the literature, specifically that it enables a dehumanization of relationships between queer men.

Users, often cited an "animal" need, beyond their understanding, to explain their desire to go without condoms. To illustrate, consider how Eric rationalizes his preference for PrEP. Eric describes his experience of engaging in condomless sex as "uninhibited, naked, exposed, raw, and utterly logically pointless, at least for anything beyond pleasure and bonding, at least among gay men." Condomless sex, for Eric, serves a social need that sex with condoms cannot. "So how does a condom fit into such conduct?" He asks, “It doesn't.” He explains, “I think the best sex involves shedding these layers (latex or otherwise) of logic and responsibility and enjoying our full animal selves along with another person we desire or better yet even admire and care for." He notes that to put on a "numbing prophylactic wrapper" completely defeats the purpose of the encounter. Eric further points out that he is not advocating condom non-use,

Of course, we all "should" use them, and I still do when I'm "being good." And the sex can still be great, even better afterward when the regret and worry don't inevitably 
follow. But I guess I came to a self-admission that there was logic in my illogical aversion to them.

Eric is outlining a contradiction in the logic of condom use and intimate encounters, he notes that for some sexual encounters, condoms are fine; but when he wants to enjoy the "full animal selves" of his partner, he cannot use a condom, as it defeats the purpose of the encounter.

This logic, as Eric makes clear, however, runs counter to theories of toxicity outlined in the above section. As he aptly notes, there is a "logic in [his] illogical aversion to them." If intimate encounters require "full" access, barriers are responsible but illogical in that they do not meet the purpose of the interaction. Constructions of contagion necessitate barriers, of course the contagion in this case is the partner themselves. This is illustrated through the nearly universal sense that queer sex is a "mistake" $(007,003)$, a "stupid" mistake $(001)$ or "regrettable" (003) to non-users. Compared to the relatively flippant attitudes of heterosexual condom non-users (e.g. Flood, 2003) and the sense of elation experienced by user participants such as Christian or Vince, there appears to be a clear divergence in the way gay men understand access to their partner when HIV is seen as a non-issue. From the above discussions, it should be fairly apparent, as 003 notes, this whole discussion has been about barriers. A sense that one must guard themselves from their partner ones, deny them full access in one or another form, in order to protect themselves from infection. A sense that to truly open themselves to their partner is a risk. In these examples, PrEP's potential is not necessarily its ability to promote life or expel death, it is in the kind of life or unlife it allows. It is no surprise then, that while heterosexuals enjoy intimacy under the false conflation of HIV virions and queer sex, queer guys understand their sexuality as inherently toxic, harmful and a mistake.

PrEP, however, does not necessarily engage with HIV as a hoard at the gates. Gilead certainly has constructed it this way in their informational videos, however, PrEP is far more 
revolutionary in its engagement. By interacting with HIV at the molecular level, it renders the virus inert. This is a very different logic than the one afforded by condoms. Condoms erect a barrier between you and your partner and thus requires one to perceive the body of one's partner as a proximal harm. PrEP on the other hand allows one to conceive of the body as nonthreatening and HIV as a harmless pathogen, among other viruses to which we are already immune.

Then the "latent need" for PrEP is perhaps an underlying need for fraternity. A familial bond established through intimate relationships. When I say intimate, I do not mean romantic or loving per se. Intimacy is a type of relationship where a person is allowed full access to another. Newmahr (2011) notes intimacy is often falsely conflated with an intimate relationships or romantic love. While it is certainly the case that romantic love can be intimate, it is not necessary for one to experience intimacy. Newmahr (2011) drawing on Simmel, writes that intimacy is often mistaken for many other types of relationships. This is especially the case because sexual practices are assumed to be a close and thus intimate relationship. Though intimacy is often conflated with romantic love, intimacy is not necessarily solely derived from romantic love. Newmahr argues that sociologically, intimacy can be understood as a social situation through which people construct an experience of closeness and access, regardless of the emotional experience of the act. Citing Dean's (2009) point that the act of fisting, Newmahr (2011) argues, is not necessarily intimate in the sense that it is immediately connected love, sex, or tenderness, it is nonetheless intimate as it allows both men to inhabit the same physical space. What is key here, is that intimacy, a relationship that requires full access to a person, is in conflict with notions of contagion that aim to protect aspects of the body from contamination. However, to lack that contact is inherently alienating as it requires you to dehumanize your partner. 
In this way, then, PrEP is not only useful in its ability to inhibit HIV virions from reproducing or in hindering the spread of virus, it is about the sense that bodies are perceived to be contaminated or are understood as a contamination in of themselves. In the sense that our lives are framed as one of intoxication, though perhaps an anticipation of intoxication, it is unclear whether or not the existence of a virion actually matters in the larger scheme. As 003 makes clear, our lives, intoxicated or not is quite clearly immaterial. For we are more saliently toxin than person. This is because animacy is not predicated on whether or not we are actually alive, but by how "holistically you are interpreted and how dynamic you are perceived to be" (Chen, 2012, p.210). In this sense PrEP's role in cleaning our blood can be understood. It is progressive in the sense that it allows us to reclaim portions of our animacy, in this case intimacy, that continued to be held captive by the legacy of GRID. Specifically, the sense that same gender sexual intercourse is inherently a risk. This sense that all gay sex is unsafe sex makes intimacy near impossible.

We have learned, then, that perhaps the "latent need," is a deep seated to recognize ourselves as human. By divorcing the disease from all queer persons, anxieties about HIV can be removed from calculations about intimacy. By removing that calculation, HIV is rendered less prominent in our conception of the self and we are able to engage more fully with our partner as a person. The interesting question that remains to be seen is, how evenly will these novel forms of intimacy be accessed? The argument so far has been that this sense that PrEP enables the privileged access to barebacking without fear of HIV (e.g. Poicus, 2016). However, it is worth noting that non-users and users alike see a future without fear of HIV that is enabled by PrEP. This future is understandable. As PrEP is a relational drug and thus infused with the various 
values and norms of the persons immersed in that user's community, it serves to reason that the dynamics of risk and immunity will be affected beyond the privilege of the user.

\section{The Changing Dynamic of HIV Risk?}

We have discussed the ontological shifts that have occurred to sexuality since the start of the HIV epidemic. AIDS synergized with the ontological status of queer men as a social contagion into a literal contagion. As much a threat to the social body as to their own. The discovery of the HIV virus and the creation of HIV tests allowed that definition of contagion to shift from all gay men to gay men whose practices were deemed unclean or dangerous. Though the management of this status was afforded to negative persons, this negation was always framed as a negation of an inevitable positive. In the sense that anybody could be contaminated, the very bodies of gay men remain toxic or potentially toxic. The imagery invoked by PrEP, a cellular barrier, a bodily barrier and a national barrier (Amico et al., 2014) exists alongside notions, like 003's, that PrEP is able to somehow clean their blood, allows us to understand the anxiety and hope that frames its role in biomedical citizenship.

From this discussion, we can conceive of new ways forward with respect to HIV prevention. PrEP allows us to understand with further clarity the less apparent problems with HIV prevention discourse. Understanding HIV prevention through the lens of PrEP users, helps us further understand the needs of these users from prevention services in Ontario. Specifically, we can understand how PrEP helps us clarify the way HIV has impacted various sexualities. Depending on how one perceives their partner: as a risk, as a fling or as a partner or as a lover, PrEP takes on a slightly different meaning. This is compounded by the epidemiological categories that further informs that risk category. These various relationships and the risk profile accorded to them speaks to the multiplicity of queer sexualities that have been universally coded 
as "at risk" and it speaks to the problems this coding has made for persons navigating their sexuality. It also speaks to future problems for HIV prevention, specifically the inadequacy of current models of epidemiological and preventative programming to adequately inform persons about their risk profiles in the event of PrEP.

This brings us to the final question on the subject of PrEP, what forms of life, what kinds of people can PrEP make emergent? Rose (2007) notes that whereas medical practice has always been about restoring the body to some predetermined norm, biomedicine is increasingly in the realm of probability and possibility. Biomedical biopolitics takes on a multiple character, filtered through the lens of personal choice and responsibility. In light of these shifts, what sorts of responsibilities will be conferred, and upon whom? While MSM continue to occupy the lion's share of new HIV diagnoses in Canada, the epidemiological picture is certainly changing. In Toronto for example, HIV diagnoses have dipped slightly for older MSM, a sign the AIDS council of Toronto has taken to be a sign that PrEP is indeed working (Aids Council of Toronto (ACT), 2016). As I mentioned above, HIV transmission is already highly regionalized in Canada. Though in the East and in BC, HIV continues to disproportionately impact MSMs, the dynamic is changing in the West. HIV is mainly endemic in heterosexual communities in Manitoba and Alberta; it is mainly endemic in injection drug using communities in Saskatchewan and makes up a near equal of diagnoses to MSMs in BC (Public Health Agency of Canada, 2014). There is further evidence that HIV is growing amongst heterosexual females and HIV continues to impact aboriginal people disproportionately more than any other ethnic group (ibid). A successful PrEP intervention into the queer community certainly aims to further complicate these dynamics of infection. PrEP stands to effectively dissolve the connection between HIV and same gender sexual identities, and frame HIV along biomedical access. As 
PrEP enters this changing space, framed as a lifestyle drug, of most use to middle class, white, gay men; the dynamics of HIV transmission in Ontario, and perhaps in Canada more generally, will certainly change. 


\section{Chapter 7}

\section{A Map}

At the outset of this thesis, I asked what it would be like to imagine what it would like to be a MSM and not be "haunted" by the looming specter of HIV. After nearly four decades conceiving of HIV as a defining fact of queer life, I wondered what would happen if there was suddenly a "magic" pill that rendered HIV inert? I wondered what that pill might do to calculations of intimacy, sexuality and fraternity between queer guys. This thesis examines the implications of such a pill. By examining the accounts of queer persons, some who use the pill, others who do not, I have attempted to sketch what this world might look like. Though PrEP is lauded for its highly effective ability to inhibit the reproductive cycle of HIV virions, evidently, PrEP interferes with more than just the spread of HIV. PrEP undermines foundational truths that structure how sex, intimacy and sexuality are understood. As we have explored, PrEP redefines immunity and redraws the boundaries of acceptable sexuality.

My participants' narratives largely echo many of the findings in other recent studies in mainly Anglophone countries. However, there were a number of new barriers to both the success of the technology and access to uses outlined in this study. The most salient of which remains the cost of treatment. Whether or not a person was able to pay for the treatment independently and discreetly was a central concern for both participant users and non-users. Confidentiality and the ability to hide PrEP use from parents or employers was a key requirement arising from both groups in some way, though unevenly so. From these accounts, it seems that it is necessary to further clarifying who constitutes a high risk of contracting HIV. As the current indications are enmeshed with notions of trust, responsibility and stigma, some would-be users decide not to take the drug. 
Stigma was a significant theme throughout the thesis. These relationships between HIV risk, acceptable sexuality and responsibility for queer guys are so pervasive they impact both the community itself and members of the health care establishment. According to users in this study, the most significant barrier to becoming a PrEP user was convincing their doctor that they were both at risk and responsible enough to use the pill. Their variety of experiences point to continued difficulty for LGBT patients to access community aware doctors. In addition to being dismissed as "unknowledgeable," users routinely interpret this dismissal as either homophobic or judgmental. Galindo et al. (2012) and Galea et al. (2011) both found this to be a significant barrier for their participants as well, indicating that without significant education on the side of health care practitioners, PrEP may never reach its target population such that it will become a useful HIV prevention technology.

As a drug that allows users to act as though they are immune to HIV, PrEP unsettles the system of acceptable sexuality that emerges in relation to HIV risk. Designed as a drug for only the "highest risk" social networks, PrEP has been interpreted by a number of groups as a drug for either the extremely vulnerable or by persons who might abuse the treatment. These characterizations neither fully understand nor adequately explain a person's motivations to become a PrEP user. In examining how participants employ prophylactics and their rationales for using those prophylaxis, I learned that HIV prevention for many MSM rests on a delicate system of relationships and symbols that are not always evenly shared within sub-communities (Adam et al., 2008). This system is more or less the same irrespective of sexuality, apart from a sense that heterosexuals who engage in sanctioned sexuality understand that they are somehow immune to HIV (Flood, 2003). While those who practice queer or unsanctioned sexuality are expected to imagine themselves as already infected. 
Should PrEP become a successful HIV prevention technology, it will no doubt shift the way HIV is conceived of by both users and non-users beyond the MSM or even the LGBT* communities. As I outlined earlier, Emtricitabine and Tenofovir both function as anti-viral medications by inhibiting the reverse transcription of HIV in infected host cells. Emtricitabine and Tenofovir halt reverse transcription by blocking the reverse transcription process by "blocking" the construction of the viral messenger RNA in the cell. Because this prophylactic method happens below the skin, at the cellular level, it exhibits properties and afford uses that condoms do not. Specifically, condoms benefit from the fairly conspicuous message that the intercourse is safe, due to the fact that it creates a barrier between partners. While this is highly effective as a method of prophylaxis and enables intercourse to happen when trust has not otherwise been established, it is problematic when trying to engage in an intimate encounter, as the logics of intimacy and contagion are not easily consolidated.

As we discovered in my second substantive chapter, PrEP stands to drastically impact the practice of serosorting. As I mention in chapter 5, how exactly each of these relationships would be impacted was beyond the scope of my study. Nonetheless, my participants noted that the acceptability of PrEP use is relationally contingent and therefore, understanding how PrEP is apt to integrate itself in those relationships warrants further research. Determining which relationships benefit most from PrEP, which ones continue to be at increased risk of HIV transmission as a result of the connotations of PrEP use in that community is certainly important for future HIV prevention planning.

While many academics have commented on the construction of PrEP users as either vulnerable or hedonistic, a problem arising from imagining the ideal users as "high risk," I have attempted to look beyond these issues into how PrEP enables novel queer futures. Thus, rather 
than interrogate the possibility of disinhibition, I examined the accounts that make up the "treatment optimism" to understand what social arrangements PrEP might enable. Though HIV is no longer necessarily a life-threatening illness, focusing on the contagion rather than the partner appears to be both a source of significant anxiety and an impediment to intimacy. PrEP is received so warmly by so many, it seems, because it means that a partner does not have to be conceived of as a contagion. Rather than perceive their partner as a possible contagion, these relationships can be understood as normal, human relationships between two consenting partners.

This is the most interesting thing about PrEP for me, personally, the implications of a paradigm where a cell can be infected while the rest of the body remains uninfected. Shifting the site of infection from the body to the cell has the simultaneous effect of shifting the boundaries of immunity, risk and HIV stigma. Rather than perceive the bodies of partners as a holistic harm to one's own entire body, HIV is less tightly tethered to stigma pervasive since the outbreak of AIDS. The divorce of HIV infection with HIV stigma allows us to understand some interesting futures for sexuality in the $21^{\text {st }}$ century. As we have seen PrEP makes possible forms of solidarity that were impractical or impossible while the body was presumed toxic.

That being said, without overcoming the barriers outlined above and in the literature more broadly, this dynamism will always be partial, incomplete and in many ways, renders the real hope for PrEP impossible. However, should we see PrEP come to be successful, we should expect some dissolution of the more stable framing divides enabled by HIV. This points to perhaps the most relevant implication for HIV prevention in Ontario. The stability of the current frame enabled by HIV, as well as corresponding health care practices are geared toward gay men as the primary risk group. This frame has implications for how straight men see themselves as at 
risk of HIV. Even without increased access to PrEP, many academics have noted that PrEP will redefine HIV risk along the lines of income, global positon and race (Pocius, 2016). As PrEP becomes further accessible to wealthy gay men it will be difficult to continue current HIV prevention discourse, especially among "straight" persons, who are not necessarily aware of their increasingly precarious HIV risk.

\section{Limitations and Areas for Further Research}

PrEP is certainly a substantive intervention into both the lives of MSM and the dynamics of HIV risk. This exploratory study has attempted to sketch as broad a picture of these dynamics within these communities as I can. Despite my best efforts to provide as rich a description as possible, these relations, by nature are transient and always in a state of becoming. Understanding the full impact of PrEP in this time and space requires far more attention than I could give in this small study. In order to remind readers of the scope of this study and the conclusions found therein, I would like to give some attention to both the limitations and gaps in the literature unresolved by this study.

Further research should aim to understand the generational experiences of PrEP users and non-users as well as the gendered experience of PrEP use. At least three of my participants identified as either trans or gender-queer. They outlined a number of challenges with respect to PrEP as a trans person. Though these are significant concerns, it is unclear exactly how pervasive these perspectives may be. Further research into the use of PrEP by trans persons will allow us to further understand the way PrEP is interpreted by trans and gender queering persons. Further, at times my participants discussed or made reference to different generations and generational interpretations of PrEP. Given that PrEP continues to be taken up slowly by younger persons, 
further understandings of generational understandings of sexuality, HIV and PrEP use might be worthwhile.

Though this paper attempts to understand portions of the HIV assemblage as they are expressed in Ontario, this assemblage is far larger than could be reasonably addressed in the scope of this paper. The most important area of complementary research, I can recommend is to attempt to comprehend how PrEP might impact heterosexual relationships. I only managed to interview one person who identified as heterosexual. Their responses at times paralleled and at other times diverged from my other respondents' own perceptions of PrEP. Given that HIV is comprehended so differently by my user and non-user groups, reproducing this study with heterosexually identified persons would be useful.

The relationship between persons of color and queer sexuality adds an entirely new dimension to queer sexuality. While I did have persons of color in my study, my questions were not designed to understand the interactions of racialization and sexuality as they are impacted by PrEP. In addition to reproducing this study in heterosexual communities, it may be worthwhile to investigate the role of PrEP in queer racialized comminutes as well as within heterosexual communities. This is imperative as "heterosexuals from HIV endemic countries" or "Hetendemic," represents a significant share of HIV diagnoses and surveillance. According to the Public Health Agency of Canada (2009) this epidemiological term "refers to a population that is largely composed of Black people of African and Caribbean descent." Further complicating the role of PrEP in new dynamics of HIV transmission, this surveillance group overlaps with the MSM and IDU group (Public Health Agency of Canada, 2009) and therefore requires further study, including how race, gender and sexuality intersect in a person's interpretation of PrEP. 
I designed this study to outline relationships between users and non-users. In doing so I also investigated how non-users became users. From these findings, I was able to make some comparisons of user's experience of becoming a user to attitudes of persons who are not users. These comparisons point to key differences between user and non-user groups as well as changing attitudes toward HIV and PrEP as a person becomes a PrEP user. However, I am reluctant to posit any directionality or causal links to my findings.

It is important to note that my user group is constructing a retrospective narrative of their experience of becoming a user. While Doucet and Mauthner (2012) argue that this narrative speaks to a narrated ontology, it is key to note that this study does not actually compare user experiences before taking PrEP, only after and in retrospect. That being said, it appears that there are substantial changes to attitudes with respect to HIV and a number of sexual relationship after taking PrEP. Though there is some evidence that PrEP does lead to some changes in sexual practices and attitudes toward sex in the literature, I cannot say that this is definitively happening as a result of PrEP or some other external factors. A study using a pre-test/post-test design may further clarify my findings and bring to light other factors that might be of use to understanding the role of PrEP in HIV prevention.

\section{Final Thoughts}

While some of these issues can be read as limitations, it may be best to imagine them as parts of the new directions for research in a rapidly changing, dynamic system. This thesis does not aim to discover, simply to describe. What I have described is a shifting landscape: a reformulation of old problems along new lines. My participants and the giants on whose shoulders I stand allow me to help chart this landscape for those who hope to build policy and further research. I have described these novel experiences being birthed from PrEP's interaction 
with established relationships not to provide the absolute final image of HIV, PrEP or queer persons, but to provide answers for those hoping to understand the practical question of what is good and bad for humans. As I have pointed out, PrEP's is precarious, unevenly accessed and generative of relations that challenge established norms and values that have held steady for nearly 40 years. PrEP makes strange bedfellows of social conservative gays and barebacking queers; it is both a sign of trustworthiness and of deceit and it changes how we will understand “safer" sex. All in all, PrEP's specific role in HIV prevention remains unclear. Frankly, one is left with a sense that PrEP demands more questions than it provides answers. Though this map provides some indication of safe passage for policy and further research, in a social world largely left uncharted. It seems that not far ahead there be dragons. 


\section{References}

Adam, B. D., Husbands, W., Murray, J., \& Maxwell, J. (2008). Silence, assent and HIV risk. Culture, Health \& Sexuality, 10(8), 759-772.

AIDS Council of Toronto. (n.d). PrEP Access in Toronto. [Presentation].

AIDS Council of Toronto. (2014). Epidemiology \& ACT's Priority Populations: Women / Gay Men / Youth. [Presentation].

Akrich, M. (1992). The De-Scription of Technical Objects. In W. Bijker \& J. Law (Eds.). Shaping technology/building society: Studies in sociotechnical change. Cambridge MA: MIT Press.

Akrich, M. \& Latour, B. (1992). A convenient vocabulary for the semiotics of human and nonhuman assemblies. In W. Bijker \& J. Law (Eds.). Shaping technology/building society: Studies in sociotechnical change. Cambridge MA: MIT Press.

Amico K., Balthazar C., Coggia T., Hosek S. (2014, June). PrEP Audio Visual Representation (PrEP REP): Development and pilot of a PrEP education video. [Presentation]. $9^{\text {th }}$ International Conference on HIV Treatment and Prevention Adherence.

Baeten, J. M., Donnell, D., Ndase, P., Mugo, N. R., Campbell, J. D., Wangisi, J., ... \& Ronald, A. (2012). Antiretroviral prophylaxis for HIV prevention in heterosexual men and women. New England Journal of Medicine, 367(5), 399-410.

Baeten, J. M., \& Grant, R. (2013). Use of antiretrovirals for HIV prevention: what do we know and what don't we know? Current HIV/AIDS Reports, 10(2), 142-151.

Benhabib, S. (1999). Sexual difference and collective identities: the new global constellation. Signs, 24(2), 335-361.

Bell, N. (2016, March 2). PrEP is now approved in Canada. What happens now? Daily Xtra.

Belluz, J. (2014). The truvada wars. BMJ: British Medical Journal, 348.

Berg, B. (2009). Qualitative research methods for the social sciences. Montreal: Allyn \& Bacon.

Bjiker (1987) The social construction of bakelite: Toward a theory of invention. In W. Bijker, P. Hughes and T.J. Pinch (Eds.), The Social Construction of Technological Systems:

New Directions in the Sociology and History of Technology. Cambridge, MA: The MIT Press

Bijker, W. E. (2010). How is technology made?-That is the question!. Cambridge journal of economics, 34(1), 63-76. 
Bowker, G., \& Star, S. L. (1999). Sorting things out: Classification and its consequences. USA: MIT Press.

Braidotti, R. (2010). The politics of "life itself" and new ways of dying. In D. Coole, \& S. Frost (Eds.). New materialisms: Ontology, agency, and politics Durham, NC: Duke University Press.

Bush, S., Magnuson, D., Rawlings, M. K., Hawkins, T., McCallister, S., \& Mera Giler, R. (2016). Racial characteristics of FTC/TDF for pre-exposure prophylaxis (PrEP) users in the US. [Conference Presentation]. ASM Microbe/ICAAC.

Calabrese, S. K., \& Underhill, K. (2015). How stigma surrounding the use of HIV preexposure prophylaxis undermines prevention and pleasure: a call to destigmatize "truvada whores". American journal of public health, 105(10), 1960-1964.

Callon, M. (1984). Some elements of a sociology of translation: domestication of the scallops and the fishermen of St Brieuc Bay. The Sociological Review, 32(S1), 196-233.

CBC News (2016, February 29/updated March 02). Truvada to prevent HIV approved in Canada. $C B C$

Chelsea and Westminster Hospital. (January 5, 2017). 42\% drop in new HIV diagnoses at 56 dean street in just 12 months [Press release].

Chen, M. (2012). Animacies. Biopolitics, Racial Mattering, and Queer Affect. Durham, NC: Duke University Press.

Collin, J. (2016). On social plasticity: the transformative power of pharmaceuticals on health, nature and identity. Sociology of health \& illness, 38(1), 73-89.

Corbett, A. M., Dickson-Gómez, J., Hilario, H., \& Weeks, M. R. (2009). A little thing called love: Condom use in high-risk primary heterosexual relationships. Perspectives on sexual and reproductive health, 41(4), 218-224.

Critelli, J. W., \& Suire, D. M. (1998). Obstacles to condom use: The combination of other forms of birth control and short-term monogamy. Journal of American College Health, 46(5), 215-219.

Dale, M. (2010). Trends in the age composition of college and university students and graduates. Education matters: Insights on education, learning and training in Canada, 7(5), Ottawa: Statistics Canada.

Dean, T. (2009). Unlimited intimacy: Reflections on the subculture of barebacking. Chicago, IL: University of Chicago Press. 
Dennin, R. H. (2016). HIV and other STI public prevention campaigns: vain endeavours to break risky new trends in view of blending sex and drugs. Progress in Health Sciences, 6(1), 158-165.

Desai, M., Iyer, G., \& Dikshit, R. K. (2012). Antiretroviral drugs: critical issues and recent advances. Indian journal of pharmacology, 44(3), 288.

Doucet, A., \& Mauthner, N. S. (2008). What can be known and how? Narrated subjects and the Listening Guide. Qualitative Research, 8(3), 399-409.

Douglas, M. (1966) Purity and danger. London: Routledge

Emans, S. J., Grace, E., Woods, E. R., Smith, D. E., Klein, K., \& Merola, J. (1987). Adolescents' compliance with the use of oral contraceptives. Jama, 257(24), 3377-3381.

Epstein, S. (1996). Impure Science: AIDS, activism and the politics of knowledge. University of California Press: London

Flood, M. (2003). Lust, trust and latex: Why young heterosexual men do not use condoms. Culture, Health \& Sexuality, 5(4), 353-369.

Flowers, P. (2001). Gay men and HIV/AIDS risk management. Health, 5(1), 50-75.

Flyvbjerg, B. (2001). Making social science matter: Why social inquiry fails and how it can succeed again. Cambridge university press.

Frankis, J., Young, I., Flowers, P., \& McDaid, L. (2016). Who will use pre-exposure prophylaxis (PrEP) and why? Understanding PrEP awareness and acceptability amongst men who have sex with men in the uk-a mixed methods study. PloS one, 11(4), e0151385.

Fraser, S., Valentine, K., \& Roberts, C. (2009). Living drugs. Science as Culture, 18(2), 123-131.

Gagnon, M., \& Holmes, D. (2016). Body-drug assemblages: theorizing the experience of side effects in the context of HIV treatment. Nursing Philosophy, 17(4), 250-261.

Galea, J. T., Kinsler, J. J., Salazar, X., Lee, S. J., Giron, M., Sayles, J. N., ... \& Cunningham, W. E. (2011). Acceptability of pre-exposure prophylaxis as an HIV prevention strategy: barriers and facilitators to pre-exposure prophylaxis uptake among at-risk Peruvian populations. International journal of STD \& AIDS, 22(5), 256-262.

Galindo, G. R., Ja'Nina, J. W., Hazelton, P., Lane, T., Steward, W. T., Morin, S. F., \& Arnold, E. A. (2012). Community member perspectives from transgender women and men who have sex with men on pre-exposure prophylaxis as an HIV prevention strategy: implications for implementation. Implementation Science, 7(1), 116. 
Gibson, J. J. (1979). The ecological approach to visual perception. New York: Psychology Press.

Gilead Sciences, Inc. (2013). Truvada: Highlights of prescribing information. Foster City, CA: Author Unknown.

Gilead Sciences, Inc. (2016). Product Monograph: Truvada. Mississauga, ON: Author Unknown.

Grant, R. M., Buchbinder, S., Cates Jr, W., \& Clarke, E. et al. (2005). Promote HIV chemoprophylaxis research, don't prevent it. Science, 309(5744), 2170.

Grant, R. M., Lama, J. R., Anderson, P. L., McMahan, V., Liu, A. Y., Vargas, L., ... \& Montoya-Herrera, O. (2010). Preexposure chemoprophylaxis for HIV prevention in men who have sex with men. New England Journal of Medicine, 2010(363), 2587-2599.

Gold, R.B. (2009, Fall). Unintended consequences: How insurance processes inadvertently abrogate patient confidentiality. Guttmacher Policy Review, 12(4), Guttmacher Institute

Golub, S. A., Gamarel, K. E., \& Surace, A. (2017). Demographic differences in PrEP-related stereotypes: implications for implementation. AIDS and Behavior, 21(5), 1229-1235.

Gupta, S. (2000). Weight gain on the combined pill--is it real?. Human reproduction update, $6(5), 427-431$.

Hacking, I. (1995). The looping effects of human kinds. Causal cognition: A multidisciplinary debate, 351-383.

Hacking, I. (2003). Risk and Dirt. In R.V. Ericson and A. Doyle (Eds), Risk and Morality (22-47). Toronto: University of Toronto Press.

Halperin, D. (2016). The biopoltics of HIV prevention discourse. In V. W. Cisney \& N. Morar (Eds.), Biopower: Foucault and beyond (pp. 199-228). Chicago, IL: The University of Chicago Press.

Hamani, Y., Sciaki-Tamir, Y., Deri-Hasid, R., Miller-Pogrund, T., Milwidsky, A., \& Haimov-Kochman, R. (2007). Misconceptions about oral contraception pills among adolescents and physicians. Human reproduction, 22(12), 3078-3083.

Harvey, D. O. (2011). Calculating risk: Barebacking, the queer male subject, and the de/formation of identity politics. Discourse, 33(2), 156-183.

Health Canada (Public Health Agency of Canada). (2015). HIV and AIDS in Canada: Surveillance report to December 31, 2014. Ottawa, Ontario: Canada. Minister of Public 
Works and Government Services Canada.

Holt, M. (2015). Configuring the users of new HIV-prevention technologies: the case of HIV pre-exposure prophylaxis. Culture, health \& sexuality, 17(4), 428-439.

Hoots, B. E., Finlayson, T., Nerlander, L., Paz-Bailey, G., Wortley, P., Todd, J., ... \& Doherty, R. (2016). Willingness to take, use of, and indications for pre-exposure prophylaxis among men who have sex with men-20 US cities, 2014. Clinical Infectious Diseases.

Hutchby, I. (2001). Technologies, texts and affordances. Sociology, 35(2), 441-456.

Hull, M.,Edward, J.,Kelley \& L.(2016, March 27). PEP/PrEP Update - A BC Case Study [Webinar]. Catie.com

Jasanoff, S. (Ed.). (2004). States of knowledge: the co-production of science and the social order. Routledge.

Jiang, J., Yang, X., Ye, L., Zhou, B., Ning, C., et al. (2014). Pre-Exposure Prophylaxis for the Prevention of HIV Infection in High Risk Populations: A MetaAnalysis of Randomized Controlled Trials. PLoS ONE, 9(2), e87674.

Joint United Nations Programme on HIV/AIDS. (2006). UNAIDS policy brief: HIV and sex between men. Geneva: UNAIDS.

Katz B.P., Fortenberry D.J., Zimet G.D., Blythe M.J. \& Orr D.P. (2000) Partner-specific relationship characteristics and condom use among people with sexually transmitted diseases. The Journal of Sex Research 37(1), 69-75.

Kingori, P., \& Sariola, S. (2015). Museum of failed HIV research. Anthropology \& Medicine, 22(3), 213-216.

Krever, H. (1997). Final report. Ottawa, Ont: Commission of Inquiry on the Blood System in Canada.

Keogh, P. (2008) Morality, responsibility and risk: Negative gay men's perceived proximity to HIV. AIDS Care, 20(5), 576-81.

Klein, J. D., Wilson, K. M., McNulty, M., Kapphahn, C., \& Collins, K. S. (1999). Access to

medical care for adolescents: results from the 1997 Commonwealth Fund Survey of the Health of Adolescent Girls. Journal of Adolescent Health, 25(2), 120-130.

Kojima, N., \& Klausner, J. D. (2016). Is emtricitabine-tenofovir disoproxil fumarate pre-exposure prophylaxis for the prevention of human immunodeficiency virus infection safer than aspirin? Open forum infectious diseases, 3(1). 
Kubicek, K., Arauz-Cuadra, C., \& Kipke, M. D. (2015). Attitudes and perceptions of biomedical HIV prevention methods: voices from young men who have sex with men. Archives of sexual behavior, 44(2), 487-497.

Lahman, M. K., Rodriguez, K. L., Moses, L., Griffin, K. M., Mendoza, B. M., \& Yacoub, W. (2015). A rose by any other name is still a rose? Problematizing pseudonyms in research. Qualitative Inquiry, 21(5), 445-453.

Latour, B. (1992). Where are the missing masses? The sociology of a few mundane artefacts. In W. Bijker and J. Law (Eds.), Shaping technology/building society: Studies in sociotechnical change (225-58). Cambridge, MA: MIT Press

Law, J. (1987). Technology and heterogeneous engineering: The case of Portuguese expansion. In W. Bijker and J. Law (Eds.), Shaping technology/building society: Studies in sociotechnical change (225-58). Cambridge, MA: MIT Press

Lehrer, J. A., Pantell, R., Tebb, K., \& Shafer, M. A. (2007). Forgone health care among US adolescents: associations between risk characteristics and confidentiality concern. Journal of Adolescent Health, 40(3), 218-226.

Marcus, G. E., \& Saka, E. (2006). Assemblage. Theory, culture \& society, 23(2-3), 101-106.

Martin, E. (1994). Flexible bodies: Tracking immunity in American culture from the days of polio to the age of AIDS. Beacon Press.

McKay, B. (2011). Lesbian, gay, bisexual, and transgender health issues, disparities, and information resources. Medical reference services quarterly, 30(4), 393-401.

McMullan, M. (2006). Patients using the Internet to obtain health information: how this affects 24-28. the patient-health professional relationship. Patient education and counseling, 63(1),

Miksche, M. (2017, January 16). Would you participate in a clinical trial just to access PrEP? Daily Xtra.

Mol, A. (2002). The body multiple: Ontology in medical practice. Durham, NC: Duke University Press.

Molina, J. M., Capitant, C., Spire, B., Pialoux, G., Cotte, L., Charreau, I., ... \& Raffi, F. (2015). On-demand preexposure prophylaxis in men at high risk for HIV-1 infection. New England Journal of Medicine, 373(23), 2237-2246.

Morgan, D. (1997). Focus groups as qualitative research. Thousand Oaks, California: Sage Publications.

Muñoz, J. E. (2009). Cruising utopia: The then and there of queer futurity. New York: NYU 
Press.

Mustanski, B., DuBois, L. Z., Prescott, T. L., \& Ybarra, M. L. (2014). A mixed-methods study of condom use and decision making among adolescent gay and bisexual males. AIDS and Behavior, 18(10), 1955-1969.

Mykhalovskiy, E. (2010) Integrating HIV treatment and prevention: Shifts in community-based organizing and biopolitics in the Canadian context. In HIV Treatment and Prevention Technologies in International Perspective. M. Davis \& C. Squire (Eds.). (167-183). New York: Palgrave Macmillan

Newmahr, S. (2011). Playing on the edge: Sadomasochism, risk, and intimacy. Indiana University Press: Bloomington, IN.

Opdenakker, R. (2006). Advantages and disadvantages of four interview techniques in qualitative research. Forum Qualitative Sozialforschung/Forum: Qualitative Social Research, 7(4).

Panel on Clinical Practices for Treatment of HIV Infection. (2002). Guidelines for the use of antiretroviral agents in HIV-infected adults and adolescents. Department of Health and Human Services: United States.

Parisi, L. (2004). Abstract sex: Philosophy, bio-technology and the mutations of desire. New York, NY: Continuum.

Patton, M. Q. (2002). Qualitative research and evaluation methods. Thousand Oaks, CA: Sage Publications Inc.

Payton, F. C., \& Kvasny, L. (2016). Online HIV awareness and technology affordance benefits for black female collegians - maybe not: the case of stigma. Journal of the American Medical Informatics Association, 23(6), 1121-1126.

Persson, A. (2012). Non/infectious corporealities: tensions in the biomedical era of 'HIV normalisation'. Sociology of Health \& Illness, 35(7), 1065-1079.

Pocius, J. (2016). Of bodies, borders, and barebacking: The geocorpographies of HIV. In Security, Race, Biopower (pp. 21-40). Palgrave Macmillan UK.

Porter, R. \& Kaplan, J. (2011). The Merck manual of diagnosis and therapy. Whitehouse Station, N.J: Merck Sharp \& Dohme Corp.

Public Health Agency of Canada. (2009). Population-specific HIV/AIDS Status Report: People from Countries where HIV is Endemic, Black People of African and Caribbean Descent Living in Canada. Ottawa: Public Health Agency of Canada.

Public Health Agency of Canada. (2014). HIV and AIDS in Canada: Surveillance Report to 
December 31st, 2011. Ottawa: Public Health Agency of Canada

Public Health Agency of Canada. (2015). HIV and AIDS in Canada: Surveillance Report to December 31st, 2014. Ottawa: Public Health Agency of Canada

Public Health Ontario. (2017). Monthly Infectious Diseases Surveillance Report 6(1) Toronto: Canada.

Public Health Ontario. (2016). Monthly Infectious Diseases Surveillance Report 5(1) Toronto: Canada.

Public Health Ontario. (2015). Monthly Infectious Diseases Surveillance Report 4(1) Toronto: Canada.

Puar, J. K. (2007). Terrorist assemblages: Homonationalism in queer times. Duke University Press.

Race, K. (2016). Reluctant objects: Sexual pleasure as a problem for HIV biomedical prevention. GLQ: A Journal of Lesbian and Gay Studies, 22(1), 1-31.

Rabinow, P (2005) Artificiality and enlightenment: From Sociobiology to Biosociality. In J.X. Inda, Athropolgies of Modernity: Foucault Governmentality and Life Politics (181-193). Malden, MA: Blackwell Publishing

Rhodes, S.D, \& Wong, F.Y. (2016). HIV prevention among diverse young MSM:

Research needs, priorities and opportunities. Aids Education \& Prevention, 28 (3), 191-201.

Roberts, T. E. \& Matthews, D. D. (2012). HIV and chemoprophylaxis, the importance of considering social structures alongside biomedical and behavioral intervention. Social Science and Medicine, 75, 1555-1561

Rose, N. (2009). The politics of life itself: Biomedicine, power, and subjectivity in the twenty-first century. Princeton, NJ: Princeton University Press.

Rose, N., \& Novas, C. (2005). Biological citizenship. In A. Ong and S. Collier (eds). Global Assemblages: Technology, Politics, and Ethics as Anthropological Problems (439-463). Malden, MA: Blackwell Publishing.

Rosengarten, M., \& Michael, M. (2009a). The performative function of expectations in translating treatment to prevention: The case of HIV pre-exposure prophylaxis, or PrEP. Social Science \& Medicine, 69(7), 1049-1055.

Rosengarten, M., \& Michael, M. (2009b). Rethinking the bioethical enactment of medically drugged bodies: Paradoxes of using anti-HIV drug therapy as a technology for prevention. Science as Culture, 18(2), 183-199. 
Rosengarten, M. \& Micheal, M. (2010). HIV Pre-exposure prophylaxis (PrEP) and the complexities of biomedical prevention: Ontological openness and the prevention assemblage. In HIV Treatment and Prevention Technologies in International Perspective. M. Davis \& C. Squire (Eds.). (167-183). New York: Palgrave Macmillan.

Rowniak, S., \& Portillo, C. (2013). Pre-exposure prophylaxis: an ethical discussion. Journal of the Association of Nurses in AIDS Care, 24(1), 6-10.

Selik, R. M., Mokotoff, E. D., Branson, B., Owen, S. M., Whitmore, S., Hall, H. I., \& Centers for Disease Control and Prevention (CDC. (2014). Revised surveillance case definition for HIV infection-United States, 2014. MMWR Recomm Rep, 63(3), 1-10.

Schoenberg, N. E., \& Ravdal, H. (2000). Using vignettes in awareness and attitudinal research. International Journal of Social Research Methodology, 3(1), 63-74.

Schot, J \& Bruheze A.A. (2003). The mediated design of products, consumption and consumers in the twentieth century. In T, Pinch \& N. Oudshoorn (eds.) How users matter: the coconstruction of users and technology (inside technology). Boston: the MIT Press.

Schwartz-Shea, P. \& Yanow, D. (2012). Interpretive research design: concepts and processes. New York, NY: Routledge.

Smith, D. K., Van Handel, M., Wolitski, R. J., Stryker, J. E., Hall, H. I., Prejean, J., ... \& Valleroy, L. A. (2015). Vital signs: estimated percentages and numbers of adults with indications for pre-exposure prophylaxis to prevent HIV acquisition-United States, 2015. MMWR Morbidity Mortality Weekly Rep, 64(46), 1291-5.

Smith Cross, J. (2016, March 01). PrEP HIV prevention could become more affordable for ontarians who need it. Metro.

Snowden, J. M., Chen, Y. H., McFarland, W., \& Raymond, H. F. (2016). Prevalence and characteristics of users of pre-exposure prophylaxis (PrEP) among men who have sex with men, San Francisco, 2014 in a cross-sectional survey: implications for disparities. Sexually Transmitted Infections, sextrans-2015.

Somers, M. R. (1994). The narrative constitution of identity: A relational and network approach. Theory and society, 23(5), 605-649.

Spieldenner, A. (2016). PrEP whores and HIV prevention: The queer communication of HIV Pre-Exposure Prophylaxis (PrEP). Journal of homosexuality, 63(12), 1685-1697.

Spinner, C. D., Boesecke, C., Zink, A., Jessen, H., Stellbrink, H. J., Rockstroh, J. K., \& Esser, S. (2016). HIV pre-exposure prophylaxis (PrEP): a review of current knowledge of oral systemic HIV PrEP in humans. Infection, 44(2), 151-158. 
Stanley, L. (1993). The knowing because experiencing subject: Narratives, lives, and autobiography. In Women's Studies International Forum. 16(3), 205-215.

Pergamon.

Stewart, D. W., \& Shamdasani, P. N. (2007). Focus groups: Theory and practice (Vol. 20). Sage publications: London.

Strapagiel, L. (2015, September 29). 5 Things Canadians Should Know About PrEP. Buzzfeed.

Tellalian, D., Maznavi, K., Bredeek, U. F., \& Hardy, W. D. (2013). Pre-exposure prophylaxis (PrEP) for HIV infection: results of a survey of HIV healthcare providers evaluating their knowledge, attitudes, and prescribing practices. AIDS patient care and STDs, 27(10), 553-559.

Treichler, P. A. (1987). AIDS, homophobia and biomedical discourse: An epidemic of signification. Cultural Studies, 1(3), 263-305.

Tuana, N (2008). Viscous porosity: Witnessing Katrina. In S. Alaimo and S. Hekman (Eds), Material Feminisms (188-213). Indiana: Indiana University Press.

Underhill, K., \& Mayer, K. H. (2013). Sexual behaviour among users of antiretroviral pre-exposure prophylaxis. The Lancet Infectious Diseases, 13(12), 996-997.

UNAIDS. (2014). The Gap Report. Joint United Nations Programme on HIV/AIDS: Geneva

van Oost, E. (2003). Materialized gender: How shavers configure the users' femininity and masculinity. In T, Pinch \& N. Oudshoorn (eds.) How users matter: the co-construction of users and technology (inside technology). (194-208) Boston: the MIT Press.

Venn, C. (2006). A note on assemblage. Theory, Culture \& Society, 23(2-3), 107-108.

Vernazza, P., Hershel B., Bernasconi, E., \& Flepp, M. (2008). HIV transmission under highly active antiretroviral therapy. Lancet, 372, 1806-7.

Watkins, E. (2011). The Construction of a Construction of a Contraceptive Technology: An Investigation of the Meanings of Norplant. Science, Technology, \& Human Values, 36(1), 33-54.

Wald, P. (2008). Contagious: cultures, carriers, and the outbreak narrative. Duke University Press.

Weeks, C. (2015, December 1). High cost of HIV drug creates 'huge equity gap.' Globe and Mail 
Woolgar, S. (1991). Configuring the user: The case of usability trials. In J. Law (ed.) A Sociology of monsters: Essays on power, technology, and domination. (58-99). New York:

Routledge.

World Health Organization. (2015a). WHO model list of essential medicines: 19th list, March 2015.

World Health Organization. (2015b). Guideline on when to start antiretroviral therapy and on pre-exposure prophylaxis for HIV. Geneva: World Health Organization.

Wyatt, S. (2003) Non-users also matter: The construction of users and non-users of the Internet. In N. Oudshoorn \& T. Pinch (Eds.), Now Users Matter: The Co-construction of Users and Technology (67-79). Cambridge, MA: MIT Press.

Yep, G. A., Lovaas, K. E., \& Pagonis, A. V. (2002). The Case of "Riding Bareback" Sexual Practices and the Paradoxes of Identity in the Era of AIDS. Journal of Homosexuality, 42(4), 1-14.

Young, I., Flowers, P., \& McDaid, L. (2016). Can a pill prevent HIV? Negotiating the biomedicalisation of HIV prevention. Sociology of health \& illness, 38(12), 411-25.

Young, I., \& McDaid, L. (2014). How acceptable are antiretrovirals for the prevention of sexually transmitted HIV?: A review of research on the acceptability of oral pre-exposure prophylaxis and treatment as prevention. AIDS and Behavior, 18(2), 195-216.

Young, J. (2007). The vertigo of late modernity. London: Sage.

Young, R. M., \& Meyer, I. H. (2005). The trouble with "MSM" and "WSW": Erasure of the sexual-minority person in public health discourse. American journal of public health, 95(7), 1144-1 


\section{Tables}

Table 1. A typology of appropriate safer sex techniques according to relationship type

\begin{tabular}{|c|c|c|c|}
\hline Relationship Type & $\begin{array}{c}\text { Neither condom } \\
\text { nor PrEP. }\end{array}$ & Condom use only & PrEP only \\
\hline $\begin{array}{c}\text { Committed } \\
\text { relationship }\end{array}$ & Appropriate & Appropriate & Dependant \\
\hline Open relationship & Inappropriate & Dependent & Appropriate \\
\hline Casual partner & Inappropriate & $\begin{array}{c}\text { Appropriate (though } \\
\text { slip ups occur) }\end{array}$ & Inappropriate \\
\hline Novel partner & Inappropriate & $\begin{array}{c}\text { Appropriate (though } \\
\text { slip ups occur) }\end{array}$ & Appropriate \\
\hline Exclusive relationship \\
$\begin{array}{c}\text { Polyamorous } \\
\text { (multiple) relationships }\end{array}$ & Appropriate & Appropriate & Inappropriate \\
\hline Clients & $\begin{array}{c}\text { Inappropriate } \\
\text { (though assault } \\
\text { may occur) }\end{array}$ & Appropriate & Appropriate \\
\hline Unfaithful partners & Inappropriate & Dependent & $\begin{array}{c}\text { Dependent (though } \\
\text { likely inappropriate) }\end{array}$ \\
\hline
\end{tabular}


APPENDIX

A. User Online Questionnaire

A.1 Recruitment 
[Text:]

Looking for interested, past or present PrEP USERS, as participants in a research project on PREP. Please email me at adamchristianson@,cmail.carleton.ca for more information or to get involved!

I am looking for people to write to me about their experiences on PrEP. IF you are interested, I will send you a form with a few questions about your experiences on PrEP, you can answer at your convenience.

I am a master's student at Carleton university looking for people who have taken or are currently taking TRUVADA for PrEP who would like to talk about their experiences.

Participants MUST be $18+$, men who have sex with men from ONTARIO, CANADA who are currently taking or who have taken PrEP.

The ethics protocol for this project has been reviewed and cleared by the Carleton University Research Ethics Board, (613-520-2517 or ethics@,carleton.ca)

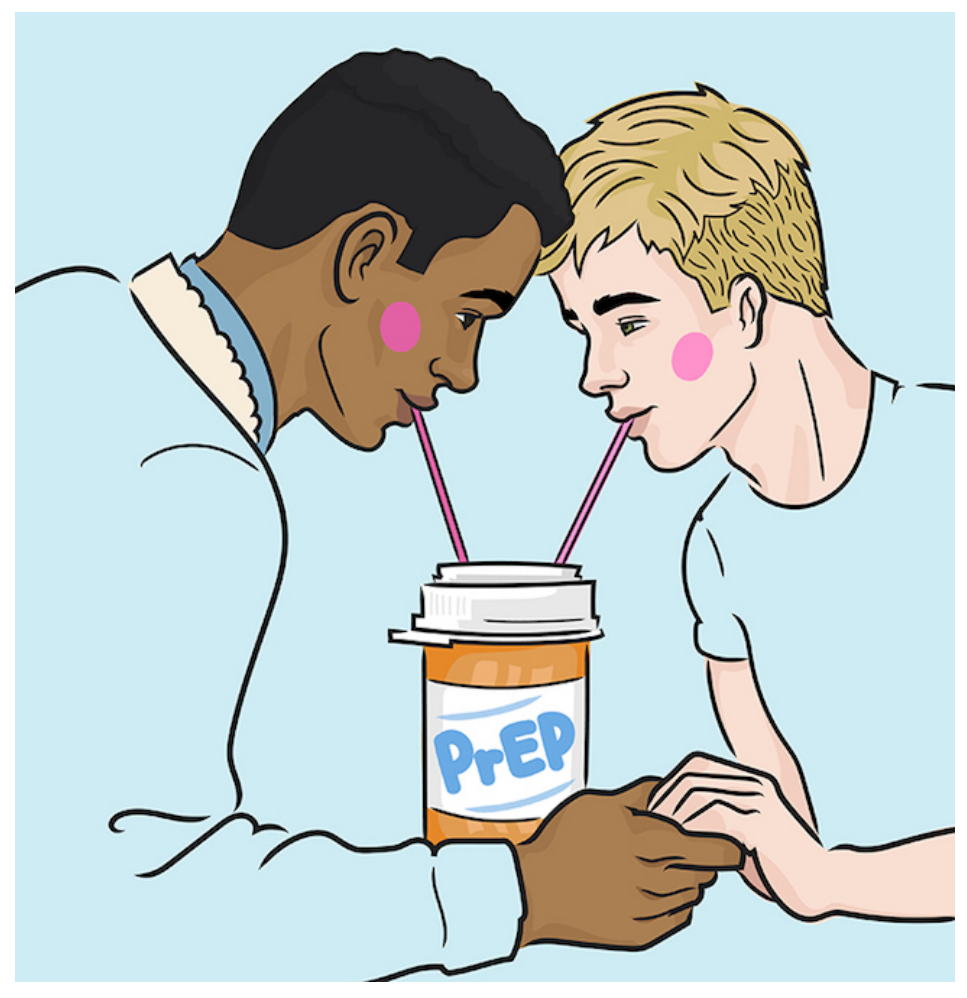




\section{A.2 User Consent Form and Questionnaire Pack}

[Running Head:]

PrEP User Online Interviews

Ethics Certificate Number:

Exp:

Participant Number: 00x

[Page]

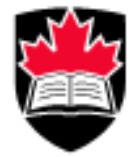

\section{Carleton \\ U N I V E R I T Y}

\section{Canada's Capital University}

PrEP User Online Interviews

Dear Participant,

Thank you for volunteering to share your experiences with me! Please read the following carefully!

You're contributing to a project that is attempting to understand how PrEP is changing the way men who have sex with men think about sex, HIV and themselves as responsible, sexually active men in Ontario. I have high hopes that by contributing to this project we are going to help health researchers and policy makers prevent the further spread of HIV.

These questions are about experiences that you have had prior to and after starting PrEP. They deal with your personal experiences taking PrEP and the experiences you have had since starting PrEP use. These are your experiences, as you remember them. Each question deals with a different experience that you may or may not have experienced since starting PrEP.

Please note that as I do not know you or your life situation, it is your job to frame these stories for an audience. This audience includes myself, Adam Christianson; my supervisor, Carlos Novas (Who can be reached at carlosnovas@carleton.ca if you have any questions) my committee and might include other students and academics in Ottawa. I will be the only person who has access to all the information you provide.

It is part of my job, and part of my commitment to learning about the effects of PrEP in the larger community, to turn my research (and your stories) into more accessible forms of information, for as many audiences as I can. In addition to my thesis, I may publish your stories in an academic journal, use it in teaching materials, at conferences or in various types of community outreach that relate to this research project. Please consider what you want these audiences to know about yourself when you are writing about these experiences. Feel free to skip questions that you think are too personal, might expose too 
much about yourself to one of these audiences or might otherwise cause you pain, harm or grief.

If you change your mind about participating or about how you answered a question after sending me this form, that is totally okay! Just let me know your participant number and the part you want to have changed or removed and I'll remove it! You may do this any time before March 1, 2017. After this date, my thesis will be in the process of being published and I will not be able to remove your responses from the study.

If you would like me to identify you, I will. Otherwise, I will do my best to keep your identity confidential and your information secured. Your stories will be kept on an encrypted disk and stored in a locked cabinet, in my home, for 5 years after the publication of my thesis and then incinerated. The only time I cannot promise to keep your identity confidential is if you indicate that you plan to harm yourself or another person.

\section{CONTACTS:}

Feel free to contact any of the following people if you have questions or concerns or would like to verify the study.

ME:

Adam Christianson, Principal researcher Master of Arts Student Department of Sociology Carleton University

\section{SUPERVISOR:}

Dr. Carlos Novas, Professor of Sociology Carleton University
CARLETON

ETHICS:

Professor Louise Heslop, Chair (CUREB-A) or Professor Shelley Brown, Chair (CUREB-B) Professor Andy Adler, Vice-Chair Carleton University Research Ethics Board carlosnovas@carleton.ca 613-520-2600 ext. 2615 Loeb D797 Dept. of Sociology and Anthropology 1125 Colonel By Drive Ottawa, Ontario K1S 5B6 ethics@carleton.ca

613-520-2517

Carleton University

Research Office, Carleton University, 511 Tory

1125 Colonel By

Drive Ottawa, Ontario K1S 5B6 
By completing the questionnaire and returning it to me, you are consenting to participate, in agreement with the terms above. Please keep a copy for your records.

Would you like to be identified (by first name only) or would you like to be given a pseudonym (a "fake name"):

(Enter Response here) 


\section{A.3 PrEP User Questionnaire}

Instructions:

I am looking for your reflections, thoughts and experiences of your everyday life now that you are taking PrEP. Think of your responses as little stories. They don't need to be long, but they should be detailed. They can also ramble away from the question if you want. Let your narrative develop naturally (also don't worry about grammar -- I don't judge).

In order to represent you and your experiences accurately, I need fairly detailed account of your stories as you experienced them. Please include as much detail about these experiences as you can remember. Try to use the following to get you started:

\section{Who was involved}

(e.g. friends, family members, professionals, strangers)

\section{What happened}

Where did this event happen?

(e.g. A doctor's office, your bedroom, a coffee shop)

\section{When did it happen}

(relative to other stories or you could use a date) and

how did you feel about it?

If you choose to answer a question, please try write at least 250 words (approximately a paragraph). There is no word limit. Fill this form out at your leisure. You don't need to fill it out all at once. I am looking for as rich an experience as you can give. If you feel tired, take a break - do the rest later. Just make sure to get the questionnaire back to me on or before October $31^{\text {st }}, 2016$.

\section{EXAMPLE: Tell me about the first time you heard about PrEP.}

When I first heard about PrEP I was really excited. I think it was mid to late 2015 (I feel like I was late to the game on this). I wasn't really paying attention to the discussion people were having about it, cause, looking back... I know a bunch of people who are taking it.

I think the first time I was really thinking about PrEP was after two events. They happened one after the other and I cant really remember which one happened first. The first one was that my friend started taking PrEP, he was advertising it on his Grindr. His tagline read something like this " On PrEP, educate yourself," this got me curious about PrEP. I guess that means that my initial reaction was curiosity - what does that "educate yourself" mean?! I mean, that's probably just my training kicking in...im always thinking about "what things mean". LMFAO I'm such a dork.

But after the second event I was definitely more excited then curious-I was watching the drag queen Wilam on YouTube and he said that he was going to start taking PrEP. He brands himself as really promiscuous, so that made sense to me. But after doing some research on PrEP, I started getting more excited. It seemed to be so effective, why wasn't everyone taking it? Was it a cure for AIDS? Would this disease that I was 
so very afraid of, finally going to end? The only real issue I thought about was that there wasn't really a subsidy for the pill — and it wasn't legally available in Canada as PrEP.

I started thinking about how I could get the pill relatively easily available for students at my school. I was excited about the pill, and I have a sense that students get aroundand they don't really use condoms. At the time my best friend had accidently gotten pregnant (later another one of my friends would get pregnant) so clearly we could use a pill that prevented HIV - I mean a baby is one thing... but HIV-jeeze.

These questions can be answered in any order:

\section{Tell me about the first time you heard about PrEP.}

2. Tell me about the moment you decided you wanted to take PrEP.

3. Tell me about how you convinced your doctor you were a suitable candidate for PrEP: (what did you need to do, where did you have to go, what hoops did you have to jump through, if any; how did this process make you feel?)

\section{How do you feel about barebacking? Has your view changed since you started taking PrEP?}

\section{How has PrEP changed your sex life.}

6. How do you feel people perceive you now that you are taking PrEP? Do they perceive you differently now that you are taking PrEP?

These questions should be answered in order:

\section{Tell me about a time when you discussed PrEP with a friend}

7a. Would you say that this story is a typical experience? Why or why not?

7b. If this experience is not typical, for what reason did you choose to share it? 
8a. Would you say that this story is a typical experience? Why or why not?

8b. If this experience is not typical, for what reason did you choose to share it?

Optional: Is there anything else you would like me to know about your experience taking PrEP?

\section{DEMOGRAPHICS AND FEEDBACK}

Thanks for participating in this questionnaire. These are just a few questions to help me keep accurate information about who participated in my study and gives you a chance to give me some feedback.

You do not need to fill this out if you do not want to. You can skip any questions you feel are too personal or that you do not feel comfortable answering.

1.How old are you today?

$18-24$

$25-34$

$35-44$

$45-54$

$55+$

2. Which of these broad racial categories would you say best describes you?

White/Caucasian

Aboriginal/Indigenous/Metis

Asian

African/ Black/Afro-Caribbean

Hispanic/Latino

Middle Eastern/Central Asian 
Other (please specify):

3. Which category best describes the approximate combined income of all the member of your household.

Less than 15000

$150000-29999$

$30000-49999$

$50000-69999$

$70-99999$

$100000+$

4. Which of the following best describes the highest level of education that you, yourself have achieved?

Some High school

High school

Some university

Some college

College Diploma

Bachelors Degree

Masters Degree

Professional degree

Doctorate

5. What is your current relationship status?

Single

Partnered - short term

Partnered - long term

Married or similar

How long have you been taking PrEP? (if you have taken a break from PrEP, add up the months together)

Less than one month

1-3 months

3-6 months

6-12 months

1-2 years

$2+$ years

6. Why were you interested in participating in this project? 
7. What would you have changed about this questionnaire or the questionnaire process?

8. If I decide to hold additional interviews with participants, would you be interested in being contacted for a follow up interview? 


\section{A.4 Debrief Form}

\section{PrEP User Debrief Package}

\section{Thank you.}

You have just participated in a project that is attempting to understand how PrEP is changing the way men who have sex with men think about sex, HIV and themselves as responsible, sexually active men in Ontario. I have high hopes that by contributing to this project we are going to help health researchers and policy makers prevent the further spread of HIV.

\section{What am I trying to learn?}

Currently vast majority of academic research on PrEP has to do with either the design and usefulness of the dug, or projected issues related to the use of the drug in the real world. Truvada (the pill used for PrEP) has only been conceived of as PrEP for a little over 5 years and there actually not a lot of research on the subject. There are especially few people writing on PrEP at this time, in my subject area. While studies on how PrEP is experienced by users is increasing rapidly, these experiences actually are not yet well understood.

The way PrEP changes interactions between friends, family or sexual partners is even further under-researched. We do not really understand the social effects of the drug in the real world. This is confounded by recent findings that, despite indications that sexually active Gay men are largely aware of PrEP, the actual take-up is fairly low. While we have some ideas about why this is the case, those suspicions have yet to be confirmed. I want to know exactly this.

\section{How have I helped you learn this?}

All the questions I have asked you to answer deal with interactions you've had with a number of different people, that may or may not have been changed by PrEP.

This research project is aiming for a more complete picture of how PrEP is changing relationships, and how pre-existing relationships might change PrEP and the dynamics of HIV, down the line. Your personal expertise, your expert knowledge of yourself and your knowledge of the intricacies of the networks of relationships that make up your life will allow me to connect with the medical literature that has been growing in the last decade. I designed this questionnaire to help shed light on how think about your self, the drug and people who don't use PrEP. You have also provided me with how you etched out your identity as a "PrEP user."

\section{What if I have a question about the project or if I want more information?}

You can contact me for further information, to ask questions or to talk about how you've experienced the study, if you want. I am also happy to hear suggestions about how to make the study safer, more inclusive or better in any other way. Alternatively, you may want to contact my supervisor. 
ME:

Adam Christianson, Principal researcher

Master of Arts Student

Department of Sociology

Carleton University

Email adamchristianson@cmail.carleton.ca

$\underline{\text { Tel }}$

Mail Loeb C777

Dept. of Sociology and Anthropology

Carleton University,

1125 Colonel By Drive

Ottawa, Ontario K1S 5B6
SUPERVISOR:

Dr. Carlos Novas, Professor of Sociology Carleton University

carlosnovas@carleton.ca

613-520-2600 ext. 2615

Loeb D797

Dept. of Sociology and Anthropology

1125 Colonel By Drive

Ottawa, Ontario K1S 5B6

\section{What If I have a concern about the project?}

I designed this project to be as painless and safe as I could, within my ability, while still answering my research question. There is still a chance, however, that something might have happened that I did not account for. If you noticed anything that concerned you, please do not hesitate to contact Professor Louise Heslop, Chair (CUREB-A) Carleton University Research Ethics Board at ethics@,carleton.ca or by telephone at 613-5202517. Together, she and I will figure out how to avoid that problem in the future.

\section{What if this discussion made me aware of something that made me Anxious, Hurt or Sad? \\ Although I tried to avoid particularly triggering subjects; HIV, PrEP and our romantic lives are sensitive subjects. Discussing these subjects might have made you aware of pain that you didn't know you had before the study. If it is the case that you are feeling distressed after completing the questionnaire, please consider accessing the Ontario Mental Health hotline, live chat, or email chat for counselling and other mental health options in your area.}

\section{Ontario Mental Health Hotline}

1-866-531-2600

LIVE CHAT

http://livechat.connexontario.ca/ECCChat/MHHchat.html

EMAIL CHAT

http://www.mentalhealthhelpline.ca/Home/Email 
B. Non-User Focus Group

B.1 Recruitment 


\section{CURIOUS ABOUT}

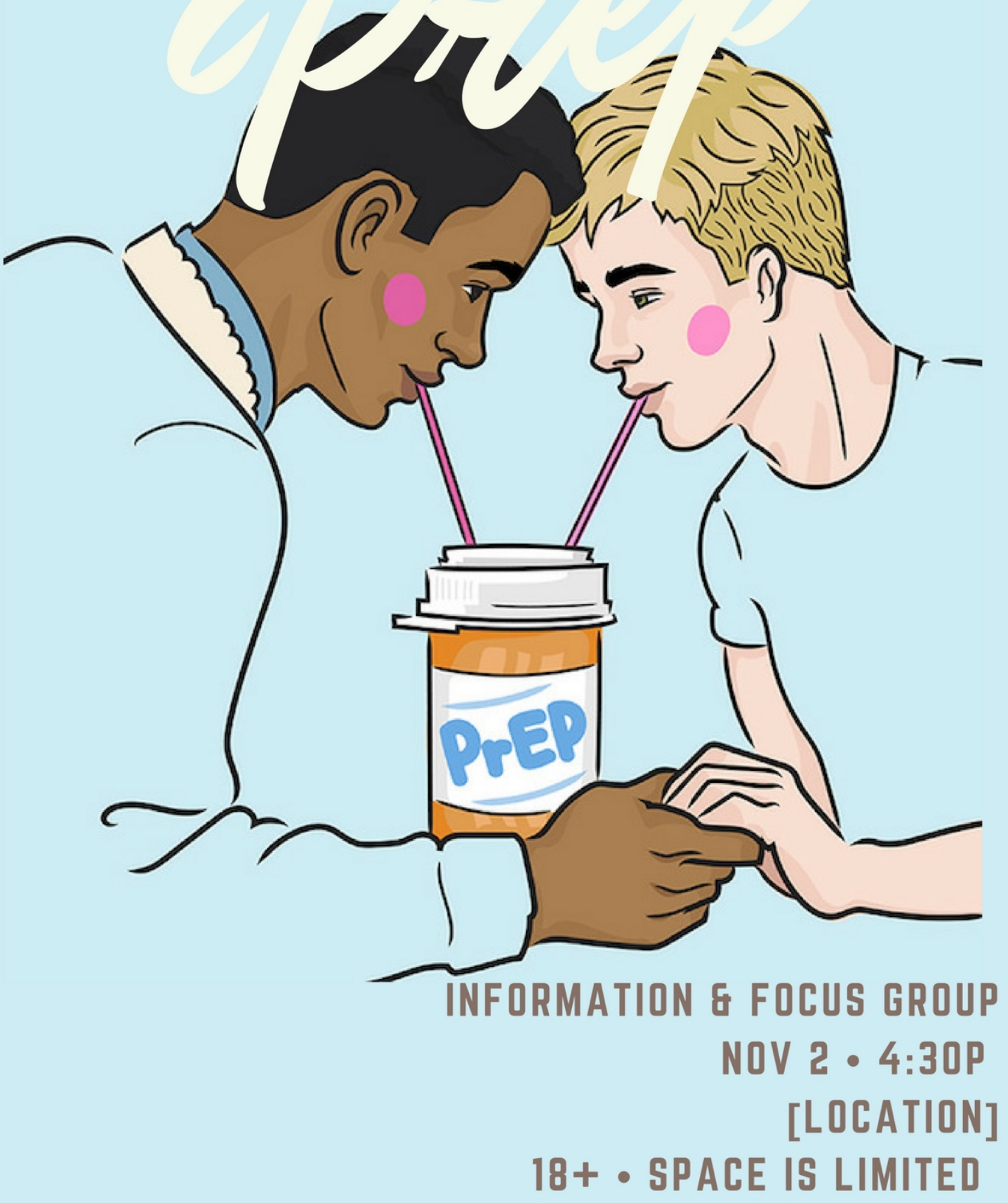

THE ETHICS PROTOCOL FOR THIS PROJECT HAS BEEN REVIEWED AND CLEARED BY THE CARLETON UNIVERSITY RESEARCH ETHICS BOARD (CUREB) 613-520-2517 OR ETHICS@्GCARLETON.CA. CONTACT: ADAMCHRISTIANSONECMAIL.CARLETON.CA FOR MORE INFORMATION. 


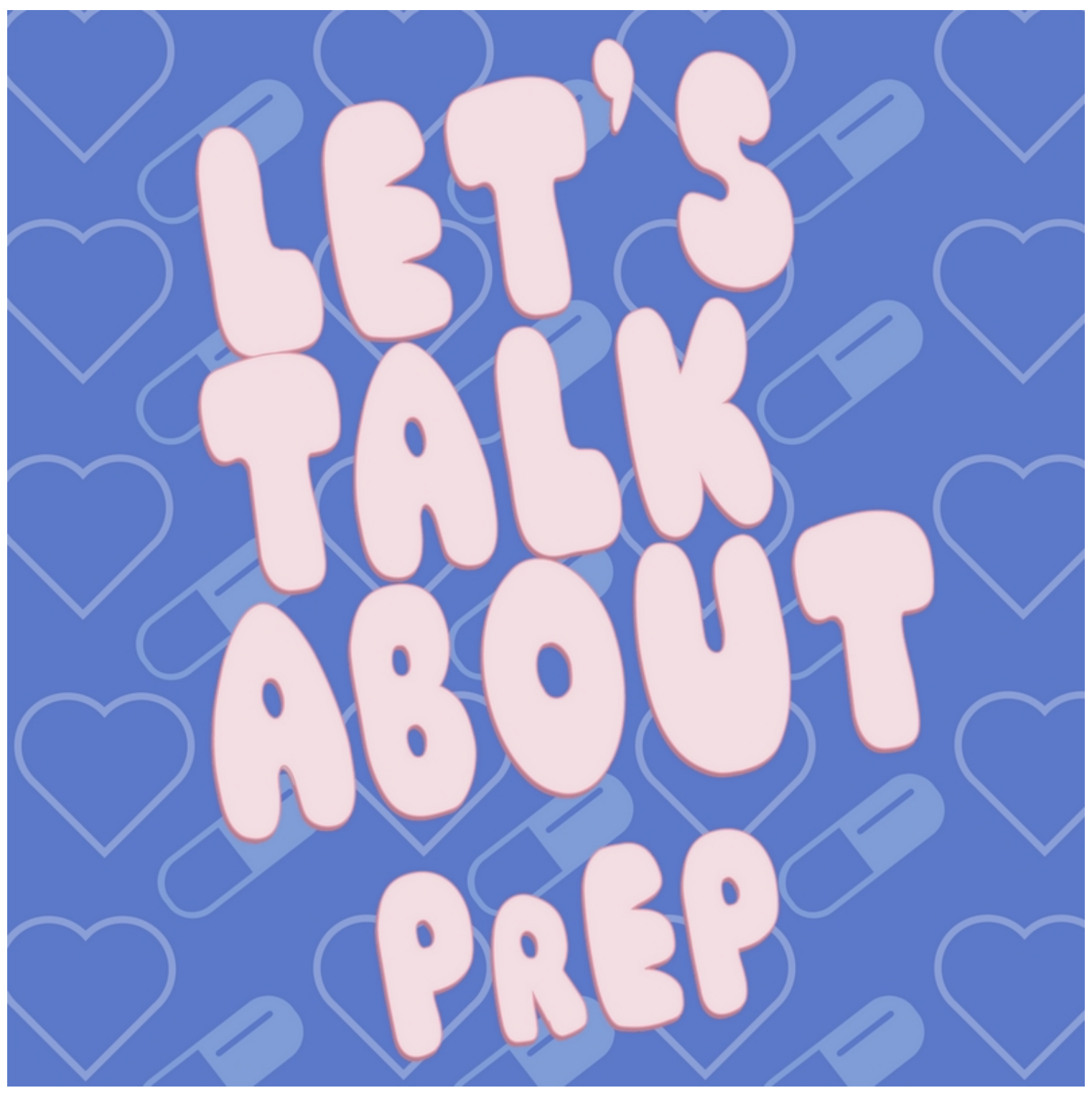




\section{CURIOUS ABOUT $P_{R} E P ?$}

\section{Come to a free info session and} focus group

NOV3@4:30PM

[LOCATION]

$18+$

( LIMITED SPACE)

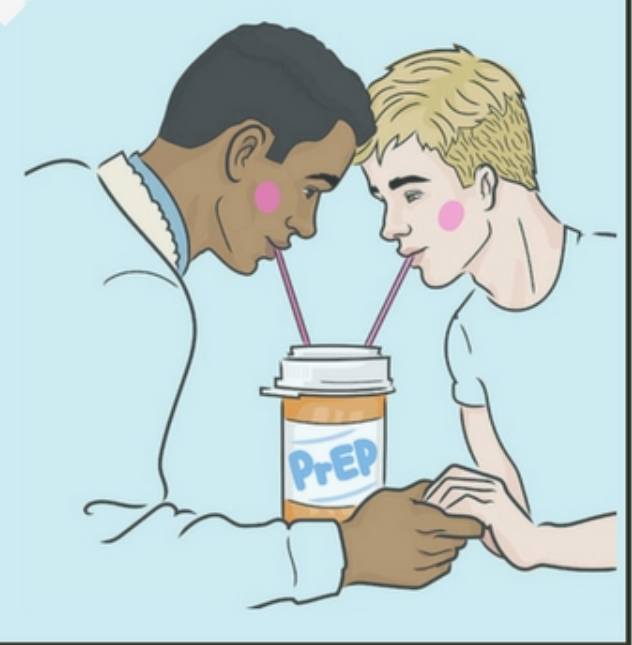

The ethics protocol for this project has been reviewed and cleared by the Carleton University Research Ethics Board (CUREB) 613-520-2517 or ethics@carleton.ca. Contact: Adam.christianson@carleton.ca for more information. 


\section{B.2 Consent Form}

[Running Head]

PrEP Non-User Focus Group

Carleton

Ethics Certificate Number:

Exp:

Canada's Capital University

\section{[Running Foot]}

Page $x$ of 2

This document has been printed on both sides of one piece of paper. Please retain a copy of this document for your records.

\section{PrEP Non-User Focus Group}

Thank you for taking the time to participate in this focus group. This focus group is part of a project that is attempting to understand how Pre-Exposure Prophylaxis is changing the way men who have sex with men think about sex, HIV and themselves as responsible, sexually active men in Ontario. I have high hopes that, by contributing to this project, we are going to help health researchers and policy makers prevent the further spread of HIV. This focus group will take about an hour and will cover how you think about PrEP, yourself and people who are taking PrEP. We will also discuss two hypothetical scenarios.

By filling out the following contract, you agree and consent to participate in the following study. If you choose to withdraw from the study, you may leave at any time during the focus group.

The ethics protocol for this project was reviewed and received ethics clearance by the Carleton University Research Ethics Board. If you would like to contact me, my supervisor or the Carleton ethics board we can be reached at the following addresses:

ME:

Adam Christianson, Principal researcher Master of Arts Student Department of Sociology Carleton University
SUPERVISOR:

Dr. Carlos Novas, Professor of Sociology Carleton University
CARLETON ETHICS:

Professor Louise Heslop, Chair (CUREB-A) Carleton University 
Research Ethics

Board

Email adamchristianson@cmail.carleton.ca Tel

Mail Loeb C777

Dept. of Sociology and Anthropology

Carleton University, 1125 Colonel By Drive Ottawa, Ontario K1S 5B6

carlosnovas@carleton.ca ethics@carleton.ca 613-520-2600 ext. 2615 613-520-2517

Loeb D797

Carleton University

Dept. of Sociology and Anthropology

Research Office, Carleton 1125 Colonel By Drive University, Ottawa, Ontario K1S 511 Tory 5B6

1125 Colonel By Drive Ottawa, Ontario K1S 5B6 
$\mathrm{I}$, , volunteer to participate in a

focus group on Pre-Exposure Prophylaxis (PrEP). I understand that I am here of my own free will and can leave whenever I choose. I am under no obligation to answer any question and can decline to answer if I choose.

\section{Study Inclusion Criteria}

I am at least 18 years of age and have not been diagnosed with HIV.

\section{Sensitive Discussion Topics}

I understand that we will be discussing topics that could be emotionally or socially sensitive, including my sexuality, condom use, HIV and any related topic that might develop from those discussions. I understand that I am under no obligation to participate in these discussions and will only participate to the extent that I am comfortable.

\section{Audio Recording}

I understand that this discussion will be recorded on an audio recording device.

\section{Use of Information}

I understand that this discussion will be recorded and used in a Masters thesis. I understand that any information from my conversation or the exit survey may end up in presentations, journal articles or community education documents. Although the researcher will do his best to keep my responses confidential and I understand that my name will be changed, I also understand that it is my job to participate and contribute only to the extent that I am comfortable providing information for these audiences.

\section{Storage and Disposal of Information}

I understand that a recording of this discussion will be stored on a password protected USB, that can be accessed solely by the researcher. This disk will be kept in a locked cabinet, alongside this form and my exit survey in the researcher's home for 5 years. After five years the disk and all files will be incinerated.

\section{Exit Survey}

I understand that at the end of this focus group I will be asked to fill out an exit survey. Participation in this survey is voluntary and subject to the same conditions as outlined in this document.

\section{Exceptions to Confidentiality}

I understand that the only time the researcher is unable to keep my identity confidential, is if I have specified that I plan to harm or have harmed another person.

By signing this document, I am acknowledging that I have read and understood the above terms and have consented to participate in accordance with them. 


\section{B. 3 Focus Group Script}

Before we get started I want to set out a few ground rules: In addition to the rules outlined in the document y'all signed, I want to specify a few rules for this room.

I want this discussion to be as safe as possible. We are talking about a really sensitive subject in our community. We are all here because we want to know more about PrEP and how to stop the spread of HIV in our community. Because of the role HIV plays in our sexuality is a sensitive topic for us, we might share things that make each other uncomfortable, and that's okay. The more we share, the better information we will have for understanding how PrEP is changing our lives. That means that being honest and authentic should not be met with judgment or anger. Shitty behavior will not be tolerated. It is okay to be uncomfortable with another person's sexuality or you may not agree with their choices, but remember that they are allowed to practice whatever sexual they choose, so try not to target each other, even if you disagree with them. IF I notice you putting another person down, I will ask you to leave, and that will be awkward for everyone, so just be mindful.

This is a mutual learning environment. We are here to learn from each-other. Ask questions if you don't understand something or if you need clarification.

If you feel like the conversation is hurting, you in any way or you are starting to feel anxious - don't ignore that feeling. If you need to talk about it, we can talk about it, or you can leave the room. Remember you are here voluntarily; I will not be mad if you choose to leave or if you want to stop talking.

This is a space for gay or similarly identified people to discuss their experiences with PrEP and to learn about the drug collectively. Please remember that each person has something to contribute and something to learn.

I will be facilitating this discussion, but I will also be participating.

\section{INTRODUCTIONS 5 minutes}

Let's start by going around by saying our name, preferred pronoun and what thing about PrEP makes you the most excited.

\section{[INTRODUCE SELF]}

\section{Think about PrEP: 10 Minutes}

Its nice to meet y'all! Now that we have talked a bit about what makes us excited about PrEP. Let's talk about what concerns us about PrEP. Try to finish this sentence: Even though I'm excited about PrEP, there are some things I'm a little unsure about. I am especially concerned about 
One of the things that I found really interesting is that PrEP stops HIV from replicating inside the cell. But the HIV still got in... How do you feel about the fact that HIV is allowed to get inside the cell?

\section{Think about self: 10 minutes}

Now that you know a bit about PrEP; you know a bit about what makes it exciting and what concerns people: Who is thinking they probably wont use PrEP in the future?

Those who aren't, why aren't you planning to use it?

Has anyone tried to get PrEP?

Were you denied PrEP? Why were you denied?

\section{Think about PrEP users: 10 minutes}

Has anyone met another person using PrEP? What were your impressions about that person?

Does anyone have similar experiences? Do you disagree.

----BREAK---- 5 Min.

\section{HYPOTHETICAL SCENARIOS: 30 minutes.}

Now I want you to imagine how you would act in three possible -totally hypothetical scenarios.

\section{Scenario 1:}

You meet up with a guy off grindr/tinder/scruff etc. He is cute and you're both clicking. It is likely that you are going to hookup. Before you undress, he tells you he is on PrEP. What do you do? bareback?

If unproductive: What do you think you would say if he said he wanted to

\section{Scenario 2:}

You've been dating a guy for a few months when he tells you that he is going to start taking PrEP.

What are your initial reactions?

\section{Scenario 3:}

You decide you are going to start take PrEP. Who do you tell first, what do you tell them?

How would you tell your sexual partner? 


\section{B.4 Exit Survey}

[running head]

[Project Title]

Ethics Certificate Number:

Exp:

SURVEY NUMBER:

Thanks for participating in this focus group. These are just a few questions to help me keep accurate information about who participated in my focus groups and gives you a chance to give me some feedback. Please fill this out and give it back to the researcher when you are done.

You do not need to fill this out if you do not want to. You can skip any questions you feel are too personal or that you do not feel comfortable answering.

1.How old are you today?

$18-24$

$25-34$

$35-44$

$45-54$

$55+$

2. Which of these broad racial categories would you say best describes you?

White/Caucasian

Aboriginal/Indigenous/Metis

Asian

African/ Black/Afro-Caribbean

Hispanic/Latino

Middle Eastern/Central Asian

Other (please specify):

3. Which category best describes the approximate combined income of all the member of your household.

Less than 15000

$150000-29999$

$30000-49999$

$50000-69999$

$70-99999$

$100000+$ 
4. Which of the following best describes the highest level of education that you, yourself have achieved?
Some High school
High school
Some university
Some college
College Diploma
Bachelors Degree
Masters Degree
Professional degree
Doctorate

5. What is your current relationship status?
Single
Partnered - short term
Partnered - long term
Married or similar

6. Why were you interested in participating in this focus group?

7. What, if anything, have you learned?

8. What would you have changed about this research process? 
9. If I decide to hold additional interviews with focus group participants, would you be interested in being contacted for a follow up interview?

9.a If so how would you like to be contacted? 


\section{B.5 Debrief Form}

\section{PrEP Non-User Focus Group Debrief Package}

\section{Thank you.}

Thank you for participating in this focus group. This focus group is part of a project that is attempting to understand how Pre-Exposure Prophylaxis is changing the way men who have sex with men think about sex, HIV and themselves as responsible, sexually active men in Ontario. I have high hopes that, by contributing to this project, we are going to help health researchers and policy makers prevent the further spread of HIV.

\section{What are you trying to learn?}

Currently vast majority of academic research on PrEP has to do with either the design or projected issues related to the use of the drug in the real world. Truvada (the pill used for PrEP) has only been conceived of as PrEP for a little over 5 years and there actually not a lot of research on the subject. There are especially few people writing on PrEP at this time, in my subject area. While studies of how PrEP is experienced by users is increasing rapidly, these experiences actually are not yet well understood. The way non-users experience themselves in relation to PrEP users is even further under-researched. How non-users and users experience PrEP mediated sexual relationships is currently unexplored. We do not really understand the social effects of the drug in the real world. This is confounded by recent findings that, despite indications that sexually active Gay men are largely aware of PrEP, the actual take-up is fairly low. While we have some ideas about why this is the case, those suspicions have yet to be confirmed.

\section{How have I helped you learn this?}

In many ways there is a lot known about how PrEP is changing the HIV epidemic. However, each of these bodies of knowledge operates separately from each-other. What this research project is aiming to do is connect those bodies of knowledge for a more complete picture of how PrEP is changing your relationships, and how your relationships might change PrEP. Your personal expertise, your expert knowledge of yourself and your knowledge of the intricacies of the networks of relationships that make up your life will allow me to connect with the medical literature that has been growing in the last decade. I designed this focus group to help shed light on how you and others think about your self, the drug and people who use PrEP.

Thank you again for participating, I hope that you have gained something from this discussion as well.

\section{The Following Pages contain contacts and useful resources!}




\section{What if I have a Question About the Project or if I Want More Information?}

Have you ever left a conversation and then thought of the perfect question, a pressing concern or an especially witty comment on the ride home? If this happens after the focus group, you can contact me for further information, to ask questions or to talk about how you've experienced the study, if you want. I am also happy to hear suggestions about how to make the study safer, more inclusive or better in any other way. Alternatively, you may want to contact my supervisor.

ME:

Adam Christianson, Principal researcher

Master of Arts Student

Department of Sociology

Carleton University

Email adamchristianson@cmail.carleton.ca

Tel

$\underline{\text { Mail }}$

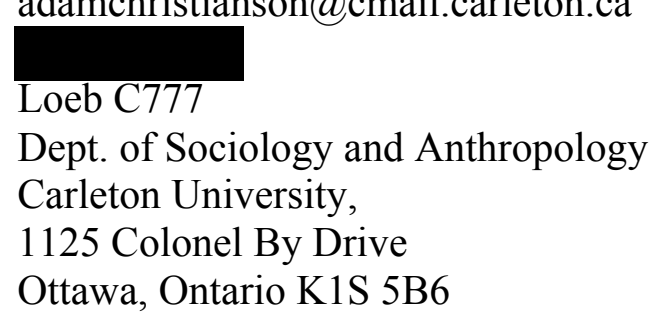

\section{SUPERVISOR:}

Dr. Carlos Novas, Professor of Sociology Carleton University

carlosnovas@carleton.ca

613-520-2600 ext. 2615

Loeb D797

Dept. of Sociology and Anthropology

1125 Colonel By Drive

Ottawa, Ontario K1S 5B6

\section{What If I have a concern about the project?}

I tried to make sure that the focus group went as smoothly as possible. However, sometimes things happen that I cannot control. There is also a chance that something happened that I did not account for. If you noticed anything that concerned you, please do not hesitate to contact Professor Louise Heslop, Chair (CUREB-A) Carleton University Research Ethics Board at ethics@carleton.ca or by telephone at 613-520-2517. Together, she and I will figure out how to avoid that problem in the future.

\section{What if this discussion made me aware of something that made me Anxious, Hurt or Sad?}

Although I tried to avoid triggering subjects; HIV, PrEP and our romantic lives are sensitive subjects. Discussing these subjects might have made you aware of pain that you didn't know you had before the study. Below is a list of a number of support services you can access if you want to manage anxiety or pain that might have come up during the course of the focus group. 


\section{FOR IMMEDIATE ASSISTANCE: \\ Ottawa Region Crisis Line:}

WITHIN OTTAWA

613.722.6914

OUTSIDE OTTAWA

1.866.996.0991

For MSM Specific resources and support services:

gayZONE / ZONEgaie

Centertown Community Health Centre, 420 Cooper Street at Bank Street.

info@gayzonegaie.ca

http://www.gayzonegaie.ca/

(613) 580-2610

\section{Walk In Counselling}

\begin{tabular}{ll}
\hline $\begin{array}{l}\text { North Renfrew Family } \\
\text { Services }\end{array}$ & $\begin{array}{l}\text { Somerset West Community } \\
\text { Health Centre }\end{array}$ \\
109 Banting Drive & 55 Eccles Street \\
(613) 584-3358 & (613) 238-8210 \\
Mondays: 9:30 am - 1:30 pm & Tuesdays: 11:00 am - 5:30 pm \\
Wednesdays: 9:30 am - 1:30 pm & (Last walk-in session is at \\
(Last walk-in session is at 12:00 & $4: 00$ pm) \\
pm) & Services available in: \\
Services available in: & Cantonese, English, Mandarin
\end{tabular}

English

\section{Family Services Ottawa \\ 312 Parkdale Avenue}

(613) 725-3601

Tuesdays: 12:00 pm - 8:00 pm

(Last walk-in session is at 6:30 pm)

Saturdays: 12:00 pm - 5:00 pm

(Last walk-in session is at 3:30 pm)

Services available in:

English

For direct referrals and intake please contact 613-725-3601 ext. 117.

\section{FOR STUDENTS ONLY}

U Ottawa Counselling:

Call or leave a message at 613-562-5200, or come in person to 100 Marie-Curie, $4^{\text {th }}$ floor.
Carleton:

To make an appointment for counselling:

For students living off campus: Main Clinic Rm. 2600 CTTC Bldg. to book in person or Call 613-520-6674

For students living in residence: Counselling is available Sept. 
to April Call 613-520-2600 ext. 8061 for intake.

\section{What if I want to educate myself further about PrEP? RESOURCES ON PrEP \\ Centers for Disease Control and Prevention (CDC). (2014). Pre-exposure prophylaxis for the prevention of HIV infection in the United States-2014: A Clinical Practice Guideline. Atlanta, 500, 67. \\ Desai, M., Iyer, G., \& Dikshit, R. K. (2012). Antiretroviral drugs: critical issues and recent advances. Indian journal of pharmacology, 44(3), 288. \\ Spinner CD, Boesecke C, Zink A, et al. HIV pre-exposure prophylaxis (PrEP): a review of current knowledge of oral systemic HIV PrEP in humans. Infection. 2016 Apr;44(2):151-8. \\ CONTACT ME IF YOU CANNOT GET A HOLD OF THESE PUBLICATIONS ON YOUR OWN}

\section{TRUVADA PRODUCT MONOGRAPH:}

http://www.gilead.ca/pdf/ca/truvada_pm_english.pdf

\section{WEBSITES:}

http://www.catie.ca/en/fact-sheets/prevention/pre-exposure-prophylaxis-prep http://www.cdc.gov/hiv/basics/prep.html https://www.youtube.com/watch?v=-Xx92whZS0o http://www.unaids.org/sites/default/files/media_asset/UNAIDS_JC2764_en.pdf AIDS and Sexual Health Info Line: 1-800-668-243 


\section{Data Analysis Strategy}

\section{Reading}

Reflexive Reading

1

Subject Tracing

2

\section{Relationships}

\section{Goal}

Tasks

"what is happening here"

Establish a reflexive account

of the overarching storyline of my respondents accounts.

This process centres our attention on the active 'I' who is telling the story.

Amplify the terms in which the respondent sees/presents themselves and highlights where the respondent might be struggling to say something.

Reading for social networks, and close and intimate relations.
Take notes on my reactions and experience

- Find themes

- Find Events

- Find Protagonists

- Find Antagonists

- Find Plots

- "How do I feel about these responses"

- Make notes of each subject change in the transcript.

I. Note I

II. Note Me

III. Note We

IV. Note You

V. Note "him/her, they, it"

Make notes of

- Interpersonal Barriers

- Allies

- Enemies

- Protagonists

\section{Location}

- This should be done in my Journal.

- Themes/events etc. should be transferred to

Nvivo for further coding.

Tracing Should be done in Nvivo.

Notes about trends or shifts should be made in my journal.

This should be done once in my Journal and reviewed once again in Nvivo 
Reading for Power
"What networks do my participants identify will be changed by prep"

Reading of interview transcripts that focuses on structured power relations and dominant ideologies that frame narratives.
- Antagonists

Basically any person other than themselves.

Should I discuss my relationship to these people? And how they seemed to relate to each other?

Make notes of

- Power relations

- Dominant Ideologies

- Social Groups they align themselves with.
This should be done once in my Journal and then coded into

Nvivo 


\section{Other}

\section{Pre-Presentation Disclaimer}

Hi folks,

My name is Adam Christianson. I am a Master's student in the Sociology and Anthropology department at Carleton University. This presentation is part of a project that I am doing on pre-exposure prophylaxis ("PrEP"). I wanted to share a little bit of what I've learned about PrEP with you, hopefully so that you will know more about it leaving here today. Hopefully this presentation will help understand PrEP when it comes up in you everyday life and may help you make informed decisions, if your partner ever chooses to use it. The main purpose of this presentation, however is to get you thinking about PrEP, in anticipation for the focus group that is happening after this presentation. This is not medical advice and should not be taken as such.

I am presenting the information that I have gathered from a number of sources about PrEP [overview of content]. Although I am fairly confident in the information that I am providing, I am not a medical expert. I am not a medical professional of any sort. I am a sociologist, studying the way this drug is changing the social relations in Gay (or similar communities.) At the end of the focus group, I will be providing links to some reputable sources for more information about prep. 
